\title{
Unequal treatment: challenging socioeconomic and living area disparities in oral rehydration therapy and continued feeding in 35 Sub-Saharan African countries
}

Gebretsadik shibre woldemedhn ( $\square$ gebretshh@gmail.com )

Addis Ababa University School of Public Health https://orcid.org/0000-0001-6671-9833

Betregiorigis Zegeye

haSET maternal and child health research program

Research

Keywords: DHS, ORT and continued feeding, inequality

Posted Date: April 16th, 2020

DOl: https://doi.org/10.21203/rs.3.rs-22588/v1

License: (a) This work is licensed under a Creative Commons Attribution 4.0 International License.

Read Full License 


\section{Abstract}

\section{Background}

While the 2030 global goal has motivated proliferation of equity-oriented studies globally, we did not find sweeping studies on disparity in Oral Rehydration Theory (ORT) and continued feeding at the level of the Sub-Saharan Africa (SSA) region. Yet, exploring the within and between-country variations of the service could help synthesize useful policy lessons that can be applied to other settings within the region. We aimed to generate evidence on inequality in use of the service at the level of SSA in order to suggest the way forward to advance the momentum already gained in the region with respect to improving coverage of this lifesaving interventions.

Method

We applied the World Health Organization's Health Equity Assessment Toolkit for the analysis. Data were obtained from Demographic and Health Surveys conducted between 2012 and 2016. Thirty-five countries were selected from different income categories in SSA. ORT and continued feeding disparity was analyzed with respect to five dimensions of inequality (wealth, education, sex, residence and subnational regions) through four measures of inequality: difference, ratio, population attributable risk and fraction.

\section{Results}

Disparity in use of ORT and continued feeding plagued SSA and consistently favored advantaged population subgroups. In 20 out of 35 countries, the service was disproportionately concentrated among children from well to do households. The inequality was strikingly pronounced in Angola, Guinea, Cameroon and Mali. We also recorded sizable variations with respect to residence and education in 23 and 11 countries. Interestingly, male and female children get the service almost equally in most countries, with only four countries (Niger, Kenya, Guinea-Bissau, and Senegal) suffered sex differentials, generally to the favor of male children.

\section{Conclusion}

In SSA, unjustified disparity around use of ORT and continued feeding is a pervasive problem that requires resolute policy response soon. Eliminating the disparity would set the region miles ahead to reaching the child health related global goals to be attained in 2030. The region might benefit from equitable economic and education policies.

\section{Results}

\section{Background}

Diarrhea is among the most common health problems affecting children worldwide (1). In 2016, diarrhea claimed the lives of between 1, 244, 073 and 2, 366, 552 people at all age groups, making it one of the 
most lethal health problems. Also, diarrhea is the fifth important cause of mortality among under-five children, with between 390894 and 504613 under-five children died of it in 2016 alone. This translates to an average mortality rate of 22.4 deaths per 100000 in all ages and 70.6 deaths per 100000 among under-five children, and the mortality rate varied hugely between and with regions. SSA hosts the highest rate of the problem, with Chad (499 deaths per 100 000), Central African Republic (384.2 deaths per 100 000 ), and Niger (376 per 100000 ) being outliers by reporting the highest mortality rate of diarrhea (2).

Globally, diarrhea had a share of nearly $9 \%$ of all deaths in children younger than five years, with female children experiencing slightly higher deaths toll on average. Worldwide, absolute number of diarrheal deaths in children aged under-five dropped by closely $57 \%$ over the last nearly two decades. However, the same success has not been made for diarrhea incidence, where it decreased by only between $10.6 \%$ and $14.8 \%$ during the same time period. In terms of morbidity, diarrhea was responsible for an average share of 74.4 million DALYs, an overwhelming $63 \%$ of which happened among children younger than 5 years (2).

The burden of diarrheal death is distributed systematically and unevenly worldwide. The reality is that not just does diarrheal deaths show marked variations between countries, but it disproportionately impacts poor and marginalized individuals within countries (3). It is fairly acceptable to recognize diarrheal related death as a key marker of inequality of health between individuals and countries. If such disparities are not to be tackled soon, they are likely to jeopardize the chance to attain child health related global goals that world countries pledge to achieve by the $2030(3,4)$. The fact that diarrhea obstructs chance of achieving the SDG is especially more conspicuous for world's poorest settings such as SSA, where the highest number of unequal countries in the world exist (5).

At country level, much more needs to be done to raise coverage of effective interventions for the poor and the most disadvantaged subpopulations as well as the entire population to decrease burden of diarrheal deaths. To this end, WHO and UNICE issued a Global Action Plan for Pneumonia and Diarrhea (GAPPD) with the intent to reduce, among other aims, mortality from diarrhea in under-five children to less than 1 per 1000 live births by 2025. The action plan outlines effective interventions to stop deaths due to diarrhea among under-five children. Oral rehydration salts (ORS) and continued feeding is among the interventions mentioned under the "treat" portion of the plan's Protect, Prevent and Treat framework $(6,7)$. It is defined as the percentage of children aged 0-59 months who had diarrhea in the two weeks prior to the survey and were treated with oral rehydration therapy - oral rehydration salts or an appropriate household solution - and continued feeding (8). ORS has been shown to reduce diarrheal deaths up to $93 \%$ (9).This means that by ensuring that the intervention reaches to all population groups, irrespective of wealth, education, place of residence or regions, stopping unnecessary death that is being caused by diarrhea is within the global community's grasp (3). I fact, addressing health inequality has been the basic tenet of the sustainable development goals since 2015 (4). In any country, health inequality monitoring aims to generate information that could be used to create effective policies, programs and practices to reduce health inequity (10). But, high-quality evidence is always necessary to inform effective interventions that end the observable disparity in use of a health indicator such as ORS. After survey of 
literatures, we found that studies that discuss the situation of disparity in use of ORS and continued feeding among children with diarrhea in SSA are lacking. WHO examined the state of inequality in ORT in several countries of low income and middle income using data collected through DHS conducted until 2013(11). However, we significantly updated the available evidence by incorporating data from more recent DHSs. The study aims to generate high quality information on status of ORT disparity in SSA and put the way forward about the possibility to challenge the unacceptable disparity. The evidence could be of help to identify subgroups of a population that require big policy attention and in so doing would potentially lead to attainment of ensuring universal reproductive, maternal and child health care target of the 2030 SDG.

\section{Methods}

\section{Data}

We used data from Demographic and Health Surveys (DHS) of 35 African countries: Ghana (2014), Algeria (2012), Angola (2015), Benin (2014), Burundi (2016), Cameron (2014), Chad (2014), Comoros (2012), Congo (2014), Democratic Republic of Congo (2013), Eswatini (2014), Ethiopia (2016), Gabon (2012), Gambia (2013), Guinea (2016), Guinea Bissau (2014), Kenya (2014), Lesotho (2014), Liberia (2013), Malawi (2015), Mali (2015), Mauritania (2015), Mozambique (2015), Namibia (2013), Niger (2012), Nigeria (2013), Rwanda (2014), Sao Tome (2014), Senegal (2014), Sierra Leone (2013), Togo (2013), Uganda (2016), United Republic of Tanzania (2015), Zambia (2013) and Zimbabwe (2014). Selection of the countries in our analysis was partly based on the fact that, they are from different country level income categories (low income, lower middle and upper middle income), and this could allow us to benchmark the disparity situation in one country in a specific income category to that of another nation in a different income category. This in turn facilitates interpretation of state of inequality in ORT in a way that could potentially lead to emergence of policy relevant suggestions. Also, we included the DHSs carried out between 2012 and 2016, as DHSs conducted until 2013 were analyzed by the WHO as mentioned in the background; we continued from where previous high quality study ends.

DHS is a nationally representative household survey conducted at regular time intervals to provide countries with updated information on a different health topics such as maternal and child health, reproductive health, fertility, nutrition, mortality, and HIV/AIDS to mention just a few. The United States Agency for International development (USAID) and each country's statistical agency cooperate to run the survey with technical support from Inner City Fund (ICF) international.

Method of DHS has been described in detail in the final DHS report of each country and readers can read relevant sections of the respective countries' DHS report to learn the methodology. Concisely, it follows a two stage stratified cluster sampling design. Big geographic areas like enumeration area (EA) are selected in the first stage though Probability Proportional to Size (PPS) approach (citation), where relatively larger EA is more likely to be selected than smaller EA. In the second stage, a pre-calculated (28 to 30 ) number of households are selected from each EA. In the selected household, eligible participants (women aged 15 
to 49 years and men 15 to 59 years) interviewed on wide range of areas using questionnaires that are comparable across countries. To capture some useful information on health topics that would be of relevant in a country only, country specific questions are also included in the model questionnaire. The similarity of the methodologies in all the DHS makes it easy for sound comparison of findings across the 35 countries.

\section{Variables}

We measured inequality for ORT and continued feeding in under-five children with diarrhea. ORT and continued feeding is the the percentage of children age under-five who had diarrhea in the two weeks prior to the survey and were treated with ORT, oral rehydration salts or any appropriate solution prepared in household. A child is said to have diarrhea if he/she has passage of three or more loose or watery stools per day (12). The ORT and continued feeding was calculated for the children who were born five years prior to the respective surveys. We disaggregated utilization of ORT and continued feeding using five dimensions of inequality which included wealth (economic status), education, residence, region and sex. While educational status is classified as no education, primary and secondary education, wealth index is classified into five categories: poorest, poor, middle, rich and richest. In all the DHSs, wealth index has been used as proxy for economic status and is computed based on household assets and possessions using a statistical technique called Principal Component Analysis (13). Residence is a binary variable classified as urban vs. rural and sex as male and female. Sub-categories of a region differ based on where which country the study is done. See Tables 6 in the result section for detail on this.

\section{Statistical analysis}

We approached investigation of the inequality in utilization of oral rehydration therapy and continued feeding in two steps. First, we disaggregated utilization of ORT and continued feeding by the aforementioned equity stratifiers. This is a preliminary, but critically important step to correctly interpret findings obtained through summary measures in the later stage of our analysis. Again as part of the first step, we also presented the population share of each subgroup alongside their respective coverage of the health indicator; this is very important in the interpretation of uncertainty intervals of point estimates. Secondly, we analyzed the inequality in oral rehydration therapy and continued feeding through calculation of four measures of inequality: Difference, Population Attributable risk (PAR), Ratio and Population Attributable Fraction (PAF). When we interpret findings produced using any of these measures, we feedback to the first step to check how the summary was estimated.

Detailed account of the methods followed in this study have been found elsewhere $(10,14)$. PAR is calculated as the difference between the estimate for the reference subgroup $y_{\text {ref }}$ and the national average $\mu:$ PAR $=y_{\text {ref }}-\mu$. The selection of the reference subgroup $y_{\text {ref }}$ depends on the type of health indicator and on the characteristics of the dimension of inequality, for which PAR is calculated. The health care indicator in this study is ORT and continued feeding and it is favorable. Thus, for residence 
and sex dimensions, $\mathrm{y}_{\text {ref }}$ refers to the subgroup with the highest estimate, which are respectively urban and male subcategories. For ordered dimensions, in our study they are wealth and education, $\mathrm{y}_{\text {ref }}$ refers to the most-advantaged subgroup (wealthiest and secondary education groups). For subnational region, $\mathrm{y}_{\text {ref }}$ refers to the subgroup with the highest estimate which are different in the two series DHSs. PAF is calculated by dividing the population attributable risk (PAR) by the national average $\mu$ and multiplying the fraction by 100: PAF $=[P A R / \mu] * 100$.

D is calculated as the difference between two subgroups: $D=y_{\text {high }}-y_{\text {low }}$. For binary dimensions (residence and sex for our study), $y_{\text {high }}$ refers to the subgroup with the highest estimate ( urban and male) and $\mathrm{y}_{\text {low }}$ to the subgroup with the lowest estimate of ORT and continued feeding ( rural and female). For ordered dimensions (wealth and education for our study), $\mathrm{y}_{\text {high }}$ refers to the most-advantaged subgroup (richest and secondary education) and $\mathrm{y}_{\mathrm{low}}$ to the most-disadvantaged subgroup (poorest and illiterate group). For subnational region, $\mathrm{y}_{\text {high }}$ refers to the subgroup with the highest estimate and $\mathrm{y}_{\text {low }}$ to the subgroup with the lowest estimate. The calculation of $R$ parallels that of $D$ except that it is a ratio of two estimates instead of subtracting one from another.

The PAR and PAF are complex measures of inequality that show a potential for improvement in the national level of a health indicator if all sub-groups are performing equally with the best performing subgroup. If there is no inequality, PAR and PAF takes the value of zero. They take positive values for favorable health intervention indicators like ORT and negative for adverse health indicators. The larger the absolute value of PAF and PAR, the larger the degree of inequality. They become zero if no further improvement can be achieved, i.e. if all subgroups have reached the same level of health or health indicator of interest as the reference subgroup.

On the other hand, Difference (D) and Ratio (R) are simple measures of inequality that show inequality between just two subgroups of an equity stratifier with two or more categories. If there is no inequality, $D$ takes the value of zero and $R$ becomes one. The further the value of $D$ and $R$ from zero and 1 , respectively, the higher the level of inequality becomes. While $D$ and PAR are absolute measures, PAF and $\mathrm{R}$ are relative measures of inequality. Complex measures are able to account for all subgroups of an equity stratifer, not just two groups unlike simple measures. This feature of complex measures of inequality makes them robust choice especially in the event of population shift. However, simple measures do not possess this property. While complex measures have the drawback of complicated interpretation and calculation, simple measures have the strength of straightforward interpretation and calculation. WHO recommends adoption of simple and complex, absolute and relative measures in a study to increase the credibility and consumption of the evidence, and we followed that recommendation (10).

We measure the statistical significance of the inequality in ORT and continued feeding through $95 \%$ Uncertainty Intervals ( $\mathrm{UI}$ ). For the disparities to be statistically significantly different between dimensions of inequality across the countries, the Uls should not overlap. We analyzed the inequality in ORS using the 
WHO's HEAT software. We accessed the latest offline version of the application from internet, and did the analysis and interpret findings in accordance with equity analysis principles contained in the WHO health equity analysis book (10).

\section{Ethical Consideration}

Ethical permissions are not required for this study since we used DHS datasets already publicly available. Ethical procedures were the responsibility of the institutions that commissioned, funded, or managed the surveys. All DHS surveys are approved by ICF international as well as an Institutional Review Board (IRB) in respective country to ensure that the protocols are in compliance with the U.S. Department of Health and Human Services regulations for the protection of human subjects.

\section{Results}

\section{Wealth based inequality}

Table 1 presents utilization of ORT and continued feeding for each subgroup of the economic status stratifier across the 35 low income, lower middle income and upper middle income SSA countries along with the population share of each. 
Table 1

utilization of ORT and continued feeding for each subgroup of the economic status stratifier across the 35 low income, lower middle income and upper middle income SSA countries

\begin{tabular}{|c|c|c|c|c|c|}
\hline Setting & Year & Subgroup & Estimate $(95 \% \mathrm{Cl})$ & Population & $\begin{array}{l}\text { National } \\
\text { Coverage }\end{array}$ \\
\hline \multirow[t]{5}{*}{ Ghana } & \multirow[t]{5}{*}{2014} & $\begin{array}{l}\text { Quintile } 1 \\
\text { (poorest) }\end{array}$ & $40.16(32.36,48.49)$ & 26.37345 & 41.5043 \\
\hline & & Quintile 2 & $43.15(34.95,51.740)$ & 25.67383 & 41.5043 \\
\hline & & Quintile 3 & $36.27(27.92,45.55)$ & 20.94055 & 41.5043 \\
\hline & & Quintile 4 & $43.33(28.31,59.68)$ & 16.25041 & 41.5043 \\
\hline & & $\begin{array}{l}\text { Quintile } 5 \\
\text { (richest) }\end{array}$ & $48.26(32.49,64.39)$ & 10.76177 & 41.5043 \\
\hline \multirow[t]{5}{*}{ Algeria } & \multirow[t]{5}{*}{2012} & $\begin{array}{l}\text { Quintile } 1 \\
\text { (poorest) }\end{array}$ & $40.09(32.73,47.93)$ & 23.93135 & 41.73203 \\
\hline & & Quintile 2 & $36.58(27.52,46.70)$ & 24.41469 & 41.73203 \\
\hline & & Quintile 3 & $40.12(32.16,48.64)$ & 18.40896 & 41.73203 \\
\hline & & Quintile 4 & $42.41(34.64,50.56)$ & 18.62111 & 41.73203 \\
\hline & & $\begin{array}{l}\text { Quintile } 5 \\
\text { (richest) }\end{array}$ & $54.15(44.00,63.97)$ & 14.6239 & 41.73203 \\
\hline \multirow[t]{5}{*}{ Angola } & \multirow[t]{5}{*}{2015} & $\begin{array}{l}\text { Quintile } 1 \\
\text { (poorest) }\end{array}$ & $27.43(22.84,32.55)$ & 19.5731 & 39.68827 \\
\hline & & Quintile 2 & $33.53(28.58,38.86)$ & 23.10405 & 39.68827 \\
\hline & & Quintile 3 & $43.05(36.10,50.28)$ & 22.36672 & 39.68827 \\
\hline & & Quintile 4 & $42.32(33.53,51.62)$ & 19.44796 & 39.68827 \\
\hline & & $\begin{array}{l}\text { Quintile } 5 \\
\text { (richest) }\end{array}$ & $56.16(46.86,65.05)$ & 15.50817 & 39.68827 \\
\hline \multirow[t]{5}{*}{ Benin } & \multirow[t]{5}{*}{2014} & $\begin{array}{l}\text { Quintile } 1 \\
\text { (poorest) }\end{array}$ & $28.05(21.70,35.42)$ & 21.3574 & 32.54792 \\
\hline & & Quintile 2 & $31.98(25.84,38.81)$ & 20.12819 & 32.54792 \\
\hline & & Quintile 3 & $31.00(24.48,38.38)$ & 22.32397 & 32.54792 \\
\hline & & Quintile 4 & $35.60(28.02,43.98)$ & 21.33978 & 32.54792 \\
\hline & & $\begin{array}{l}\text { Quintile } 5 \\
\text { (richest) }\end{array}$ & $37.70(28.43,47.96)$ & 14.85066 & 32.54792 \\
\hline
\end{tabular}




\begin{tabular}{|c|c|c|c|c|c|}
\hline Setting & Year & Subgroup & Estimate $(95 \% \mathrm{Cl})$ & Population & $\begin{array}{l}\text { National } \\
\text { Coverage }\end{array}$ \\
\hline \multirow[t]{5}{*}{ Burundi } & \multirow[t]{5}{*}{2016} & $\begin{array}{l}\text { Quintile } 1 \\
\text { (poorest) }\end{array}$ & $31.65(27.61,36.00)$ & 24.07388 & 34.95439 \\
\hline & & Quintile 2 & $32.21(28.14,36.57)$ & 23.54476 & 34.95439 \\
\hline & & Quintile 3 & $37.66(33.02,42.53)$ & 20.69663 & 34.95439 \\
\hline & & Quintile 4 & $37.69(33.03,42.60)$ & 18.96193 & 34.95439 \\
\hline & & $\begin{array}{l}\text { Quintile } 5 \\
\text { (richest) }\end{array}$ & $37.76(32.52,43.30)$ & 12.7228 & 34.95439 \\
\hline \multirow[t]{5}{*}{ Cameroon } & \multirow[t]{5}{*}{2014} & $\begin{array}{l}\text { Quintile } 1 \\
\text { (poorest) }\end{array}$ & $29.41(22.99,36.77)$ & 36.38363 & 39.29078 \\
\hline & & Quintile 2 & $37 \cdot 54(31 \cdot 76,43 \cdot 70)$ & 24.52436 & 39.29078 \\
\hline & & Quintile 3 & $47.46(39.76,55.29)$ & 19.50633 & 39.29078 \\
\hline & & Quintile 4 & $48.74(40.43,57.13)$ & 11.29913 & 39.29078 \\
\hline & & $\begin{array}{l}\text { Quintile } 5 \\
\text { (richest) }\end{array}$ & $55.68(45.82,65.10)$ & 8.286552 & 39.29078 \\
\hline \multirow[t]{5}{*}{ Chad } & \multirow[t]{5}{*}{2014} & $\begin{array}{l}\text { Quintile } 1 \\
\text { (poorest) }\end{array}$ & $24.78(20.69,29.38)$ & 22.99905 & 28.88636 \\
\hline & & Quintile 2 & $26.2(21.84,31.28)$ & 21.46346 & 28.88636 \\
\hline & & Quintile 3 & $30.54(26.08,35.41)$ & 20.63436 & 28.88636 \\
\hline & & Quintile 4 & $29.64(24.54,35.31)$ & 18.16671 & 28.88636 \\
\hline & & $\begin{array}{l}\text { Quintile } 5 \\
\text { (richest) }\end{array}$ & $34.97(30.83,39.35)$ & 16.73641 & 28.88636 \\
\hline \multirow[t]{5}{*}{ Comoros } & \multirow[t]{5}{*}{2012} & $\begin{array}{l}\text { Quintile } 1 \\
\text { (poorest) }\end{array}$ & $42.44(29.79,56.16)$ & 21.86407 & 53.94333 \\
\hline & & Quintile 2 & $52.14(38.79,65.19)$ & 26.10099 & 53.94333 \\
\hline & & Quintile 3 & $53.81(43.76,63.56)$ & 21.8048 & 53.94333 \\
\hline & & Quintile 4 & $70.43(57.13,80.98)$ & 15.80697 & 53.94333 \\
\hline & & $\begin{array}{l}\text { Quintile } 5 \\
\text { (richest) }\end{array}$ & $56.76(43.31,69.27)$ & 14.42317 & 53.94333 \\
\hline \multirow[t]{3}{*}{ Congo } & \multirow[t]{3}{*}{2014} & $\begin{array}{l}\text { Quintile } 1 \\
\text { (poorest) }\end{array}$ & $36.33(32.17,40.71)$ & 26.99686 & 42.24259 \\
\hline & & Quintile 2 & $37.76(31.53,44.42)$ & 22.61654 & 42.24259 \\
\hline & & Quintile 3 & $46.79(38.79,54.95)$ & 23.70311 & 42.24259 \\
\hline
\end{tabular}




\begin{tabular}{|c|c|c|c|c|c|}
\hline Setting & Year & Subgroup & Estimate (95\% Cl) & Population & $\begin{array}{l}\text { National } \\
\text { Coverage }\end{array}$ \\
\hline & & Quintile 4 & $48.67(38.10,59.36)$ & 15.47905 & 42.24259 \\
\hline & & $\begin{array}{l}\text { Quintile } 5 \\
\text { (richest) }\end{array}$ & $47.01(34.83,59.55)$ & 11.20444 & 42.24259 \\
\hline \multirow[t]{5}{*}{$\begin{array}{l}\text { Democratic Republic of } \\
\text { the Congo }\end{array}$} & \multirow[t]{5}{*}{2013} & $\begin{array}{l}\text { Quintile } 1 \\
\text { (poorest) }\end{array}$ & $32.22(27.70,37.10)$ & 22.11872 & 39.94501 \\
\hline & & Quintile 2 & $41.93(36.12,47.98)$ & 20.3818 & 39.94501 \\
\hline & & Quintile 3 & $42.45(36.89,48.21)$ & 17.5361 & 39.94501 \\
\hline & & Quintile 4 & $41.13(32.75,50.07)$ & 21.77431 & 39.94501 \\
\hline & & $\begin{array}{l}\text { Quintile } 5 \\
\text { (richest) }\end{array}$ & $43.24(36.99,49.72)$ & 18.18908 & 39.94501 \\
\hline \multirow[t]{5}{*}{ Eswatini } & \multirow[t]{5}{*}{2014} & $\begin{array}{l}\text { Quintile } 1 \\
\text { (poorest) }\end{array}$ & $45.38(35.66,55.47)$ & 24.64696 & 46.37177 \\
\hline & & Quintile 2 & $42.35(33.10,52.18)$ & 25.42505 & 46.37177 \\
\hline & & Quintile 3 & $59.32(47.64,70.03)$ & 21.65247 & 46.37177 \\
\hline & & Quintile 4 & $40.04(23.45,59.27)$ & 17.34297 & 46.37177 \\
\hline & & $\begin{array}{l}\text { Quintile } 5 \\
\text { (richest) }\end{array}$ & $42.30(24.44,62.43)$ & 10.93255 & 46.37177 \\
\hline \multirow[t]{5}{*}{ Ethiopia } & \multirow[t]{5}{*}{2016} & $\begin{array}{l}\text { Quintile } 1 \\
\text { (poorest) }\end{array}$ & $23.84(17.54,31.53)$ & 20.66412 & 28.65617 \\
\hline & & Quintile 2 & $34.24(25.29,44.47)$ & 23.14477 & 28.65617 \\
\hline & & Quintile 3 & $28.52(21.26,37.09)$ & 21.78754 & 28.65617 \\
\hline & & Quintile 4 & $25.52(16.47,37.32)$ & 20.64595 & 28.65617 \\
\hline & & $\begin{array}{l}\text { Quintile } 5 \\
\text { (richest) }\end{array}$ & $31.38(21.22,43.70)$ & 13.75762 & 28.65617 \\
\hline \multirow[t]{5}{*}{ Gabon } & \multirow[t]{5}{*}{2012} & $\begin{array}{l}\text { Quintile } 1 \\
\text { (poorest) }\end{array}$ & $53.44(46.83,59.93)$ & 23.05507 & 51.90965 \\
\hline & & Quintile 2 & $46.54(36.24,57.15)$ & 22.88687 & 51.90965 \\
\hline & & Quintile 3 & $52.79(41.63,63.67)$ & 25.56293 & 51.90965 \\
\hline & & Quintile 4 & $56.01(41.00,70.00)$ & 19.25994 & 51.90965 \\
\hline & & $\begin{array}{l}\text { Quintile } 5 \\
\text { (richest) }\end{array}$ & $50.36(30.35,70.25)$ & 9.235194 & 51.90965 \\
\hline
\end{tabular}




\begin{tabular}{|c|c|c|c|c|c|}
\hline Setting & Year & Subgroup & Estimate (95\% Cl) & Population & $\begin{array}{l}\text { National } \\
\text { Coverage }\end{array}$ \\
\hline \multirow[t]{5}{*}{ Gambia } & \multirow[t]{5}{*}{2013} & $\begin{array}{l}\text { Quintile } 1 \\
\text { (poorest) }\end{array}$ & $45.46(35.70,55.58)$ & 18.60457 & 50.84321 \\
\hline & & Quintile 2 & $48.78(40.93,56.68)$ & 22.01013 & 50.84321 \\
\hline & & Quintile 3 & $54.75(47.19,62.09)$ & 19.14623 & 50.84321 \\
\hline & & Quintile 4 & $56.77(48.68,64.52)$ & 20.97492 & 50.84321 \\
\hline & & $\begin{array}{l}\text { Quintile } 5 \\
\text { (richest) }\end{array}$ & $48.04(36.23,60.08)$ & 19.26415 & 50.84321 \\
\hline \multirow[t]{5}{*}{ Guinea } & \multirow[t]{5}{*}{2016} & $\begin{array}{l}\text { Quintile } 1 \\
\text { (poorest) }\end{array}$ & $32.55(25.30,40.75)$ & 23.38568 & 44.17994 \\
\hline & & Quintile 2 & $46.59(37.20,56.22)$ & 21.31853 & 44.17994 \\
\hline & & Quintile 3 & $37.88(29.63,46.90)$ & 21.44252 & 44.17994 \\
\hline & & Quintile 4 & $48.71(39.90,57.60)$ & 17.28433 & 44.17994 \\
\hline & & $\begin{array}{l}\text { Quintile } 5 \\
\text { (richest) }\end{array}$ & $60.89(51.64,69.43)$ & 16.56895 & 44.17994 \\
\hline \multirow[t]{5}{*}{ Guinea-Bissau } & \multirow[t]{5}{*}{2014} & $\begin{array}{l}\text { Quintile } 1 \\
\text { (poorest) }\end{array}$ & $48.29(40.19,56.50)$ & 20.4378 & 54.61463 \\
\hline & & Quintile 2 & $50.44(41.34,59.52)$ & 18.95342 & 54.61463 \\
\hline & & Quintile 3 & $55.97(47.37,64.23)$ & 20.75555 & 54.61463 \\
\hline & & Quintile 4 & $59.24(48.25,69.38)$ & 25.1164 & 54.61463 \\
\hline & & $\begin{array}{l}\text { Quintile } 5 \\
\text { (richest) }\end{array}$ & $58.91(49.28,67.90)$ & 14.73684 & 54.61463 \\
\hline \multirow[t]{5}{*}{ Kenya } & \multirow[t]{5}{*}{2014} & $\begin{array}{l}\text { Quintile } 1 \\
\text { (poorest) }\end{array}$ & $39.21(35.39,43.16)$ & 26.97726 & 45.1324 \\
\hline & & Quintile 2 & $43.17(38.66,47.79)$ & 22.85396 & 45.1324 \\
\hline & & Quintile 3 & $44.55(38.79,50.46)$ & 18.44987 & 45.1324 \\
\hline & & Quintile 4 & $49.88(43.87,55.89)$ & 17.77935 & 45.1324 \\
\hline & & $\begin{array}{l}\text { Quintile } 5 \\
\text { (richest) }\end{array}$ & $54.51(47.23,61.61)$ & 13.93956 & 45.1324 \\
\hline \multirow[t]{3}{*}{ Lesotho } & \multirow[t]{3}{*}{2014} & $\begin{array}{l}\text { Quintile } 1 \\
\text { (poorest) }\end{array}$ & $50.74(38.34,63.05)$ & 23.5475 & 61.38574 \\
\hline & & Quintile 2 & $58.67(45.27,70.90)$ & 22.94458 & 61.38574 \\
\hline & & Quintile 3 & $63.59(50.86,74.66)$ & 20.5444 & 61.38574 \\
\hline
\end{tabular}




\begin{tabular}{|c|c|c|c|c|c|}
\hline Setting & Year & Subgroup & Estimate (95\% Cl) & Population & $\begin{array}{l}\text { National } \\
\text { Coverage }\end{array}$ \\
\hline & & Quintile 4 & $74.18(58.20,85.56)$ & 18.49361 & 61.38574 \\
\hline & & $\begin{array}{l}\text { Quintile } 5 \\
\text { (richest) }\end{array}$ & $63.51(44.54,79.04)$ & 14.46991 & 61.38574 \\
\hline \multirow[t]{5}{*}{ Liberia } & \multirow[t]{5}{*}{2013} & $\begin{array}{l}\text { Quintile } 1 \\
\text { (poorest) }\end{array}$ & $40.90(34.20,47.96)$ & 27.80155 & 46.43533 \\
\hline & & Quintile 2 & $46.12(39.69,52.68)$ & 23.096 & 46.43533 \\
\hline & & Quintile 3 & $40.22(33.34,47.50)$ & 18.49649 & 46.43533 \\
\hline & & Quintile 4 & $56.40(47.37,65.02)$ & 18.8366 & 46.43533 \\
\hline & & $\begin{array}{l}\text { Quintile } 5 \\
\text { (richest) }\end{array}$ & $53.91(40.07,67.17)$ & 11.76936 & 46.43533 \\
\hline \multirow[t]{5}{*}{ Malawi } & \multirow[t]{5}{*}{2015} & $\begin{array}{l}\text { Quintile } 1 \\
\text { (poorest) }\end{array}$ & $55.52(51.36,59.61)$ & 25.49789 & 56.68572 \\
\hline & & Quintile 2 & $52.71(48.71,56.67)$ & 23.42749 & 56.68572 \\
\hline & & Quintile 3 & $58.30(53.79,62.69)$ & 18.02529 & 56.68572 \\
\hline & & Quintile 4 & $58.00(53.33,62.53)$ & 17.38706 & 56.68572 \\
\hline & & $\begin{array}{l}\text { Quintile } 5 \\
\text { (richest) }\end{array}$ & $61.17(53.64,68.21)$ & 15.66228 & 56.68572 \\
\hline \multirow[t]{5}{*}{ Mali } & \multirow[t]{5}{*}{2015} & $\begin{array}{l}\text { Quintile } 1 \\
\text { (poorest) }\end{array}$ & $22.87(18.05,28.53)$ & 22.17164 & 28.70292 \\
\hline & & Quintile 2 & $23.73(19.26,28.86)$ & 23.23614 & 28.70292 \\
\hline & & Quintile 3 & $27.42(22.49,32.99)$ & 20.08137 & 28.70292 \\
\hline & & Quintile 4 & $32.73(26.86,39.20)$ & 18.74054 & 28.70292 \\
\hline & & $\begin{array}{l}\text { Quintile } 5 \\
\text { (richest) }\end{array}$ & $41.04(35.12,47.24)$ & 15.77031 & 28.70292 \\
\hline \multirow[t]{5}{*}{ Mauritania } & \multirow[t]{5}{*}{2015} & $\begin{array}{l}\text { Quintile } 1 \\
\text { (poorest) }\end{array}$ & $29.11(24.50,34.19)$ & 26.2099 & 34.50784 \\
\hline & & Quintile 2 & $29.46(25.27,34.04)$ & 27.03965 & 34.50784 \\
\hline & & Quintile 3 & $41.39(35.90,47.10)$ & 20.9822 & 34.50784 \\
\hline & & Quintile 4 & $40.05(33.33,47.17)$ & 16.64085 & 34.50784 \\
\hline & & $\begin{array}{l}\text { Quintile } 5 \\
\text { (richest) }\end{array}$ & $38.98(29.33,49.57)$ & 9.12741 & 34.50784 \\
\hline
\end{tabular}




\begin{tabular}{|c|c|c|c|c|c|}
\hline Setting & Year & Subgroup & Estimate (95\% Cl) & Population & $\begin{array}{l}\text { National } \\
\text { Coverage }\end{array}$ \\
\hline \multirow[t]{5}{*}{ Mozambique } & \multirow[t]{5}{*}{2015} & $\begin{array}{l}\text { Quintile } 1 \\
\text { (poorest) }\end{array}$ & $28.06(18.00,40.93)$ & 23.96414 & 43.02846 \\
\hline & & Quintile 2 & $39.37(28.76,51.08)$ & 25.08219 & 43.02846 \\
\hline & & Quintile 3 & $48.54(35.87,61.40)$ & 20.93607 & 43.02846 \\
\hline & & Quintile 4 & $60.70(48.43,71.75)$ & 16.14818 & 43.02846 \\
\hline & & $\begin{array}{l}\text { Quintile } 5 \\
\text { (richest) }\end{array}$ & $46.59(36.45,57.02)$ & 13.86943 & 43.02846 \\
\hline \multirow[t]{5}{*}{ Namibia } & \multirow[t]{5}{*}{2013} & $\begin{array}{l}\text { Quintile } 1 \\
\text { (poorest) }\end{array}$ & $50.30(42.25,58.34)$ & 28.4082 & 51.98061 \\
\hline & & Quintile 2 & $54.44(45.82,62.81)$ & 23.81028 & 51.98061 \\
\hline & & Quintile 3 & $50.37(42.12,58.60)$ & 22.05145 & 51.98061 \\
\hline & & Quintile 4 & $57.02(47.05,66.46)$ & 16.40956 & 51.98061 \\
\hline & & $\begin{array}{l}\text { Quintile } 5 \\
\text { (richest) }\end{array}$ & $45.68(31.24,60.89)$ & 9.320512 & 51.98061 \\
\hline \multirow[t]{5}{*}{ Niger } & \multirow[t]{5}{*}{2012} & $\begin{array}{l}\text { Quintile } 1 \\
\text { (poorest) }\end{array}$ & $33.29(26.22,41.19)$ & 18.80169 & 42.96736 \\
\hline & & Quintile 2 & $42.07(33.83,50.79)$ & 19.04196 & 42.96736 \\
\hline & & Quintile 3 & $44.06(36.99,51.37)$ & 20.24891 & 42.96736 \\
\hline & & Quintile 4 & $46.83(40.56,53.19)$ & 23.01669 & 42.96736 \\
\hline & & $\begin{array}{l}\text { Quintile } 5 \\
\text { (richest) }\end{array}$ & $47.60(41.43,53.85)$ & 18.89076 & 42.96736 \\
\hline \multirow[t]{5}{*}{ Nigeria } & \multirow[t]{5}{*}{2013} & $\begin{array}{l}\text { Quintile } 1 \\
\text { (poorest) }\end{array}$ & $16.43(13.44,19.93)$ & 28.04678 & 29.23584 \\
\hline & & Quintile 2 & $25.88(22.51,29.57)$ & 26.07713 & 29.23584 \\
\hline & & Quintile 3 & $34.69(29.02,40.84)$ & 18.50417 & 29.23584 \\
\hline & & Quintile 4 & $37.51(31.18,44.30)$ & 15.16292 & 29.23584 \\
\hline & & $\begin{array}{l}\text { Quintile } 5 \\
\text { (richest) }\end{array}$ & $47.23(40.61,53.94)$ & 12.209 & 29.23584 \\
\hline \multirow[t]{3}{*}{ Rwanda } & \multirow[t]{3}{*}{2014} & $\begin{array}{l}\text { Quintile } 1 \\
\text { (poorest) }\end{array}$ & $18.53(13.68,24.61)$ & 29.05479 & 19.46708 \\
\hline & & Quintile 2 & $17.59(12.63,23.95)$ & 25.62788 & 19.46708 \\
\hline & & Quintile 3 & $20.12(14.66,26.97)$ & 18.91029 & 19.46708 \\
\hline
\end{tabular}




\begin{tabular}{|c|c|c|c|c|c|}
\hline Setting & Year & Subgroup & Estimate (95\% Cl) & Population & $\begin{array}{l}\text { National } \\
\text { Coverage }\end{array}$ \\
\hline & & Quintile 4 & $23.03(16.42,31.30)$ & 14.8677 & 19.46708 \\
\hline & & $\begin{array}{l}\text { Quintile } 5 \\
\text { (richest) }\end{array}$ & $20.31(13.06,30.20)$ & 11.53934 & 19.46708 \\
\hline \multirow[t]{5}{*}{ Sao Tome and Principe } & \multirow[t]{5}{*}{2014} & $\begin{array}{l}\text { Quintile } 1 \\
\text { (poorest) }\end{array}$ & $63.70(52.01,73.97)$ & 24.57356 & 61.02909 \\
\hline & & Quintile 2 & 69.18(58.35,78.24) & 25.02055 & 61.02909 \\
\hline & & Quintile 3 & $50.86(35.49,66.07)$ & 15.9972 & 61.02909 \\
\hline & & Quintile 4 & $56.27(43.49,68.27)$ & 25.59041 & 61.02909 \\
\hline & & $\begin{array}{l}\text { Quintile } 5 \\
\text { (richest) }\end{array}$ & $62.68(40.22,80.75)$ & 8.818278 & 61.02909 \\
\hline \multirow[t]{5}{*}{ Senegal } & \multirow[t]{5}{*}{2014} & $\begin{array}{l}\text { Quintile } 1 \\
\text { (poorest) }\end{array}$ & $52.81(46.24,59.28)$ & 27.18121 & 48.59681 \\
\hline & & Quintile 2 & $50.32(44.33,56.29)$ & 21.1315 & 48.59681 \\
\hline & & Quintile 3 & $46.93(39.24,54.78)$ & 17.29135 & 48.59681 \\
\hline & & Quintile 4 & $37.46(25.85,50.73)$ & 18.27034 & 48.59681 \\
\hline & & $\begin{array}{l}\text { Quintile } 5 \\
\text { (richest) }\end{array}$ & $53.61(44.13,62.84)$ & 16.12561 & 48.59681 \\
\hline \multirow[t]{5}{*}{ Sierra Leone } & \multirow[t]{5}{*}{2013} & $\begin{array}{l}\text { Quintile } 1 \\
\text { (poorest) }\end{array}$ & $57.62(50.33,64.60)$ & 22.22783 & 57.57013 \\
\hline & & Quintile 2 & $60.08(53.52,66.30)$ & 22.29513 & 57.57013 \\
\hline & & Quintile 3 & $54.14(46.04,62.03)$ & 20.87473 & 57.57013 \\
\hline & & Quintile 4 & $56.03(48.36,63.42)$ & 19.55251 & 57.57013 \\
\hline & & $\begin{array}{l}\text { Quintile } 5 \\
\text { (richest) }\end{array}$ & $60.50(50.90,69.35)$ & 15.04979 & 57.57013 \\
\hline \multirow[t]{5}{*}{ Togo } & \multirow[t]{5}{*}{2013} & $\begin{array}{l}\text { Quintile } 1 \\
\text { (poorest) }\end{array}$ & $40.94(35.38,46.75)$ & 28.48436 & 33.42139 \\
\hline & & Quintile 2 & $32.95(26.88,39.65)$ & 24.30891 & 33.42139 \\
\hline & & Quintile 3 & $26.22(19.61,34.11)$ & 20.28351 & 33.42139 \\
\hline & & Quintile 4 & $27.85(19.42,38.20)$ & 15.24718 & 33.42139 \\
\hline & & $\begin{array}{l}\text { Quintile } 5 \\
\text { (richest) }\end{array}$ & $35.79(25.33,47.80)$ & 11.67604 & 33.42139 \\
\hline
\end{tabular}




\begin{tabular}{|c|c|c|c|c|c|}
\hline Setting & Year & Subgroup & Estimate $(95 \% \mathrm{Cl})$ & Population & $\begin{array}{l}\text { National } \\
\text { Coverage }\end{array}$ \\
\hline \multirow[t]{5}{*}{ Uganda } & \multirow[t]{5}{*}{2016} & $\begin{array}{l}\text { Quintile } 1 \\
\text { (poorest) }\end{array}$ & $34.12(30.36,38.09)$ & 25.49751 & 37.38395 \\
\hline & & Quintile 2 & $33.69(29.79,37.82)$ & 22.57913 & 37.38395 \\
\hline & & Quintile 3 & $37.10(32.42,42.03)$ & 19.02055 & 37.38395 \\
\hline & & Quintile 4 & $39.31(34 \cdot 10,44.78)$ & 16.46589 & 37.38395 \\
\hline & & $\begin{array}{l}\text { Quintile } 5 \\
\text { (richest) }\end{array}$ & $45.89(39.81,52.11)$ & 16.43692 & 37.38395 \\
\hline \multirow[t]{5}{*}{$\begin{array}{l}\text { United Republic of } \\
\text { Tanzania }\end{array}$} & \multirow[t]{5}{*}{2015} & $\begin{array}{l}\text { Quintile } 1 \\
\text { (poorest) }\end{array}$ & $36.89(30.29,44.02)$ & 18.46905 & 45.45398 \\
\hline & & Quintile 2 & $45.18(37.37,53.25)$ & 20.84221 & 45.45398 \\
\hline & & Quintile 3 & $49.36(40.11,58.65)$ & 19.80412 & 45.45398 \\
\hline & & Quintile 4 & $47.24(38.70,55.95)$ & 20.98889 & 45.45398 \\
\hline & & $\begin{array}{l}\text { Quintile } 5 \\
\text { (richest) }\end{array}$ & $47.89(38.41,57.52)$ & 19.89573 & 45.45398 \\
\hline \multirow[t]{5}{*}{ Zambia } & \multirow[t]{5}{*}{2013} & $\begin{array}{l}\text { Quintile } 1 \\
\text { (poorest) }\end{array}$ & $51.68(45.86,57.46)$ & 22.3005 & 56.17617 \\
\hline & & Quintile 2 & $50.66(45.96,55.36)$ & 21.90483 & 56.17617 \\
\hline & & Quintile 3 & $57.75(51.77,63.50)$ & 20.3411 & 56.17617 \\
\hline & & Quintile 4 & $66.16(59.93,71.87)$ & 21.10007 & 56.17617 \\
\hline & & $\begin{array}{l}\text { Quintile } 5 \\
\text { (richest) }\end{array}$ & $54.63(45.61,63.36)$ & 14.35349 & 56.17617 \\
\hline \multirow[t]{5}{*}{ Zimbabwe } & \multirow[t]{5}{*}{2014} & $\begin{array}{l}\text { Quintile } 1 \\
\text { (poorest) }\end{array}$ & $48.26(42.74,53.82)$ & 22.88103 & 56.36424 \\
\hline & & Quintile 2 & $57.50(52.22,62.61)$ & 22.07989 & 56.36424 \\
\hline & & Quintile 3 & $55.22(49.40,60.91)$ & 17.80418 & 56.36424 \\
\hline & & Quintile 4 & $62.28(56.87,67.41)$ & 25.47947 & 56.36424 \\
\hline & & $\begin{array}{l}\text { Quintile } 5 \\
\text { (richest) }\end{array}$ & $58.87(51.45,65.90)$ & 11.75543 & 56.36424 \\
\hline
\end{tabular}

In the vast majority of the studied countries, ORT and continued feeding is higher among the richest subgroups. There are also few countries with disproportionately higher coverage in quintiles 3 and 4 . For instance, in Mozambique and Lesotho, the average coverage among quintile 4 is higher than among quintile 5. In the other few countries, coverage is higher among the poorest subgroup than among the 
richest subgroup. For instance, the coverage is, on average, higher among the poorest subgroups in Togo, Namibia, Gabon and Eswatini (Table 1).

The summary measures showed considerable absolute and relative wealth-based inequality in 20 countries, and just half of which saw disparity by all the four measures. However, 15 countries did not see any economic status inequality: Burundi, Comoros, Eswatini, Ethiopia, Gabon, Gambia, Guinea Bissau, Lesotho, Namibia, Rwanda, Sao Tome, Sierra Leone, Togo, Tanzania and Zambia. Of all the countries with economic inequality, the highest, at least on average, wealth related inequality was observed in Nigeria, Angola, Guinea and Cameroon. The difference in point estimates between the richest and poorest subgroups, showed that there were nearly 31 percentage point (pp) in Nigeria, nearly 29 pp in Angola, $28 \mathrm{pp}$ in Guinea and 26 pp in Cameroon (Table 2). 
Table 2

wealth-related inequality in ORT and continued feeding in the 35 low income, lower middle income and upper middle income SSA countries

\begin{tabular}{|c|c|c|c|c|c|}
\hline \multirow[t]{2}{*}{ Country } & \multirow[t]{2}{*}{ Year } & \multicolumn{4}{|c|}{ Type and value of inequality measure with $95 \% \mathrm{Cl}$} \\
\hline & & D & $\mathbf{R}$ & PAR & PAF \\
\hline Ghana & 2014 & $\begin{array}{l}8.10(-10.22 \\
26.44)\end{array}$ & $\begin{array}{l}1.20(0.72, \\
1.67)\end{array}$ & $\begin{array}{l}6.76(0.37 \\
13.15)\end{array}$ & $\begin{array}{l}16.29(0.89 \\
31.70)\end{array}$ \\
\hline Algeria & 2012 & $\begin{array}{l}14.06(1.39 \\
26.72)\end{array}$ & $\begin{array}{l}1.35(0.99 \\
1.71)\end{array}$ & $\begin{array}{l}12.42(7.79 \\
17.05)\end{array}$ & $\begin{array}{l}29.77(18.67, \\
40.87)\end{array}$ \\
\hline Angola & 2015 & $\begin{array}{l}28.73(18.35 \\
39.11)\end{array}$ & $\begin{array}{l}2.04(1.55 \\
2.54)\end{array}$ & $\begin{array}{l}16.48(12.38 \\
20.57)\end{array}$ & $\begin{array}{l}41.52(31.19, \\
51.85)\end{array}$ \\
\hline Benin & 2014 & $\begin{array}{l}9.64(-2.37, \\
21.66)\end{array}$ & $\begin{array}{l}1.34(0.86 \\
1.82)\end{array}$ & $\begin{array}{l}5.15(0.26 \\
10.03)\end{array}$ & $\begin{array}{l}15.83(0.82 \\
30.84)\end{array}$ \\
\hline Burundi & 2016 & $\begin{array}{l}6.10(-0.72 \\
12.93)\end{array}$ & $\begin{array}{l}1.19(0.96 \\
1.42)\end{array}$ & $\begin{array}{l}2.80(-0.23 \\
5.85)\end{array}$ & $\begin{array}{l}8.03(-0.68 \\
16.75)\end{array}$ \\
\hline Cameroon & 2014 & $\begin{array}{l}26.26(14.34 \\
38.18)\end{array}$ & $\begin{array}{l}1.89(1.33 \\
2.44)\end{array}$ & $\begin{array}{l}16.38(13.11 \\
19.66)\end{array}$ & $\begin{array}{l}41.71(33.38 \\
50.04)\end{array}$ \\
\hline Chad & 2014 & $\begin{array}{l}10.18(4.10 \\
16.27)\end{array}$ & $\begin{array}{l}1.41(1.11 \\
1.71)\end{array}$ & $\begin{array}{l}6.08(3.49, \\
8.67)\end{array}$ & $\begin{array}{l}21.07(12.11 \\
30.03)\end{array}$ \\
\hline Comoros & 2012 & $\begin{array}{l}14.31(-4.48 \\
33.12)\end{array}$ & $\begin{array}{l}1.33(0.81 \\
1.86)\end{array}$ & $\begin{array}{l}2.81(-5.37 \\
11.00)\end{array}$ & $\begin{array}{l}5.22(-9.96 \\
20.40)\end{array}$ \\
\hline Congo & 2014 & $\begin{array}{l}10.67(-2.61 \\
23.96)\end{array}$ & $\begin{array}{l}1.29(0.91 \\
1.67)\end{array}$ & $\begin{array}{l}4.77(0.83, \\
8.70)\end{array}$ & $\begin{array}{l}11.29(1.98 \\
20.60)\end{array}$ \\
\hline $\begin{array}{l}\text { Democratic Republic of } \\
\text { the Congo }\end{array}$ & 2013 & $\begin{array}{l}11.02(3.09 \\
18.94)\end{array}$ & $\begin{array}{l}1.34(1.06 \\
1.62)\end{array}$ & $\begin{array}{l}3.30(0.03 \\
6.57)\end{array}$ & $\begin{array}{l}8.26(0.08 \\
16.45)\end{array}$ \\
\hline Eswatini & 2014 & $\begin{array}{l}-3.08(-25.30 \\
19.14)\end{array}$ & $\begin{array}{l}0.93(0.44 \\
1.41)\end{array}$ & $0(-8.17,8.17)$ & $\begin{array}{l}0(-17.63 \\
17.63)\end{array}$ \\
\hline Ethiopia & 2016 & $\begin{array}{l}7.54(-5.79 \\
20.88)\end{array}$ & $\begin{array}{l}1.31(0.70 \\
1.92)\end{array}$ & $\begin{array}{l}2.73(-2.04 \\
7.50)\end{array}$ & $\begin{array}{l}9.53(-7.12 \\
26.18)\end{array}$ \\
\hline Gabon & 2012 & $\begin{array}{l}-3.08(-25.11 \\
18.94)\end{array}$ & $\begin{array}{l}0.94(0.53 \\
1.35)\end{array}$ & $0(-6.46,6.46)$ & $\begin{array}{l}0(-12.45 \\
12.45)\end{array}$ \\
\hline Gambia & 2013 & $\begin{array}{l}2.58(-13.12 \\
18.29)\end{array}$ & $\begin{array}{l}1.05(0.70 \\
1.410\end{array}$ & $0(-5.64,5.64)$ & $\begin{array}{l}0(-11.11 \\
11.11)\end{array}$ \\
\hline Guinea & 2016 & $\begin{array}{l}28.34(16.51 \\
40.17)\end{array}$ & $\begin{array}{l}1.87(1.34 \\
2.39)\end{array}$ & $\begin{array}{l}16.72(10.47 \\
22.96)\end{array}$ & $\begin{array}{l}37.84(23.70 \\
51.98)\end{array}$ \\
\hline Guinea-Bissau & 2014 & $\begin{array}{l}10.61(-1.83 \\
23.06)\end{array}$ & $\begin{array}{l}1.21(0.93 \\
1.50)\end{array}$ & $\begin{array}{l}4.29(-2.17 \\
10.77)\end{array}$ & $\begin{array}{l}7.87(-3.98, \\
19.72)\end{array}$ \\
\hline
\end{tabular}




\begin{tabular}{|c|c|c|c|c|c|}
\hline \multirow[t]{2}{*}{ Country } & \multirow[t]{2}{*}{ Year } & \multicolumn{4}{|c|}{ Type and value of inequality measure with $95 \% \mathrm{Cl}$} \\
\hline & & D & $\mathbf{R}$ & PAR & PAF \\
\hline Kenya & 2014 & $\begin{array}{l}15.30(7.09 \\
23.51)\end{array}$ & $\begin{array}{l}1.39(1.16 \\
1.62)\end{array}$ & $\begin{array}{l}9.38(6.40 \\
12.36)\end{array}$ & $\begin{array}{l}20.79(14.18 \\
27.3) 9\end{array}$ \\
\hline Lesotho & 2014 & $\begin{array}{l}12.77(-8.99 \\
34.53)\end{array}$ & $\begin{array}{l}1.25(0.78, \\
1.71)\end{array}$ & $\begin{array}{l}2.12(-7.46 \\
11.72)\end{array}$ & $\begin{array}{l}3.46(-12.16 \\
19.10)\end{array}$ \\
\hline Liberia & 2013 & $\begin{array}{l}13.00(-2.45 \\
28.46)\end{array}$ & $\begin{array}{l}1.31(0.91 \\
1.72)\end{array}$ & $\begin{array}{l}7.47(3.18 \\
11.77)\end{array}$ & $\begin{array}{l}16.10(6.85 \\
25.35)\end{array}$ \\
\hline Malawi & 2015 & $\begin{array}{l}5.64(-2.75 \\
14.05)\end{array}$ & $\begin{array}{l}1.10(0.94 \\
1.25)\end{array}$ & $\begin{array}{l}4.49(1.71 \\
7.27)\end{array}$ & $\begin{array}{l}7.92(3.01 \\
12.82)\end{array}$ \\
\hline Mali & 2015 & $\begin{array}{l}\text { 18.17(10.15, } \\
26.18)\end{array}$ & $\begin{array}{l}1.79(1.30 \\
2.28)\end{array}$ & $\begin{array}{l}12.34(9.10 \\
15.58)\end{array}$ & $\begin{array}{l}43.00(31.70 \\
54.29)\end{array}$ \\
\hline Mauritania & 2015 & $\begin{array}{l}9.86(-1.44 \\
21.17)\end{array}$ & $\begin{array}{l}1.33(0.92, \\
1.75)\end{array}$ & $\begin{array}{l}4.47(1.13 \\
7.80)\end{array}$ & $\begin{array}{l}12.96(3.30 \\
22.62)\end{array}$ \\
\hline Mozambique & 2015 & $\begin{array}{l}\text { 18.53(3.02, } \\
34.03)\end{array}$ & $\begin{array}{l}1.66(0.88 \\
2.43)\end{array}$ & $\begin{array}{l}3.56(-3.18 \\
10.32)\end{array}$ & $\begin{array}{l}8.29(-7.40 \\
23.99)\end{array}$ \\
\hline Namibia & 2013 & $\begin{array}{l}-4.61(-21.85 \\
12.61)\end{array}$ & $\begin{array}{l}0.90(0.57 \\
1.24)\end{array}$ & $0(-5.50,5.50)$ & $\begin{array}{l}0(-10.59 \\
10.59)\end{array}$ \\
\hline Niger & 2012 & $\begin{array}{l}14.31(4.56 \\
24.07)\end{array}$ & $\begin{array}{l}1.43(1.05 \\
1.80)\end{array}$ & $\begin{array}{l}4.64(-0.04 \\
9.32)\end{array}$ & $\begin{array}{l}10.80(-0.09 \\
21.70)\end{array}$ \\
\hline Nigeria & 2013 & $\begin{array}{l}30.79(23.36 \\
38.23)\end{array}$ & $\begin{array}{l}2.87(2.17 \\
3.57)\end{array}$ & $\begin{array}{l}17.99(15.71 \\
20.28)\end{array}$ & $\begin{array}{l}61.55(53.73 \\
69.37)\end{array}$ \\
\hline Rwanda & 2014 & $\begin{array}{l}1.78(-8.32 \\
11.89)\end{array}$ & $\begin{array}{l}1.09(0.53 \\
1.65)\end{array}$ & $\begin{array}{l}0.85(-3.10 \\
4.80)\end{array}$ & $\begin{array}{l}4.36(-15.96 \\
24.70)\end{array}$ \\
\hline Sao Tome and Principe & 2014 & $\begin{array}{l}-1.01(-24.85 \\
22.82)\end{array}$ & $\begin{array}{l}0.98(0.61 \\
1.35)\end{array}$ & $\begin{array}{l}1.65(-7.12 \\
10.44)\end{array}$ & $\begin{array}{l}2.71(-11.67 \\
17.11)\end{array}$ \\
\hline Senegal & 2014 & $\begin{array}{l}0.80(-10.62 \\
12.23)\end{array}$ & $\begin{array}{l}1.01(0.79, \\
1.23)\end{array}$ & $\begin{array}{l}5.01(0.24 \\
9.79)\end{array}$ & $\begin{array}{l}10.32(0.50 \\
20.15)\end{array}$ \\
\hline Sierra Leone & 2013 & $\begin{array}{l}2.87(-8.84 \\
14.60)\end{array}$ & $\begin{array}{l}1.04(0.84, \\
1.25)\end{array}$ & $\begin{array}{l}2.93(-2.30 \\
8.16)\end{array}$ & $\begin{array}{l}5.09(-3.99 \\
14.18)\end{array}$ \\
\hline Togo & 2013 & $\begin{array}{l}-5.15(-17.84 \\
7.54)\end{array}$ & $\begin{array}{l}0.87(0.57 \\
1.170\end{array}$ & $\begin{array}{l}2.37(-2.53 \\
7.28)\end{array}$ & $\begin{array}{l}7.10(-7.58 \\
21.80)\end{array}$ \\
\hline Uganda & 2016 & $\begin{array}{l}11.77(4.49, \\
19.05)\end{array}$ & $\begin{array}{l}1.34(1.10, \\
1.58)\end{array}$ & $\begin{array}{l}8.51(5.50 \\
11.52)\end{array}$ & $\begin{array}{l}22.77(14.72, \\
30.83)\end{array}$ \\
\hline $\begin{array}{l}\text { United Republic of } \\
\text { Tanzania }\end{array}$ & 2015 & $\begin{array}{l}10.99(-0.85 \\
22.84)\end{array}$ & $\begin{array}{l}1.29(0.94 \\
1.65)\end{array}$ & $\begin{array}{l}2.43(-3.57 \\
8.44)\end{array}$ & $\begin{array}{l}5.36(-7.86 \\
18.58)\end{array}$ \\
\hline Zambia & 2013 & $\begin{array}{l}2.95(-7.72 \\
13.62)\end{array}$ & $\begin{array}{l}1.05(0.84, \\
1.26)\end{array}$ & $0(-4.05,4.05)$ & $0(-7.22,7.22)$ \\
\hline
\end{tabular}




\begin{tabular}{|llllll|}
\hline \multirow{2}{*}{ Country } & Year & \multicolumn{4}{l|}{ Type and value of inequality measure with 95\% Cl } \\
\cline { 3 - 6 } & & D & R & PAR & PAF \\
\hline Zimbabwe & 2014 & $10.60(1.46$, & $1.21(1.01$, & $2.50(-2.10$, & $4.44(-3.73$, \\
& & $19.74)$ & 1.420 & $7.11)$ & $12.62)$ \\
\hline
\end{tabular}

\section{Educational Inequality}

Eswatini, Senegal, Sierra Leone, Togo and Gambia had the average coverage highest among noneducation group. In other 7 countries (Ghana, Algeria, Comoros, DR Congo, Ethiopia, Gabon and Liberia), the average coverage is higher among the primary group. In Rwanda, we observed unusual concentration of the service, where the coverage is highest among no education, followed by primary and secondary. In the remaining countries, it is higher among children born into women who completed the secondary education (Table 3). 
Table 3

utilization of ORT and continued feeding for each subgroup of the education status stratifier across the 35 low income, lower middle income and upper middle income SSA countries

\begin{tabular}{|c|c|c|c|c|c|}
\hline Country & Year & Subgroup & $\begin{array}{l}\text { Estimate (95\% } \\
\mathrm{Cl})\end{array}$ & Population & $\begin{array}{l}\text { National } \\
\text { Coverage }\end{array}$ \\
\hline \multirow[t]{3}{*}{ Ghana } & \multirow[t]{3}{*}{2014} & No education & $\begin{array}{l}38.82(30.10 \\
48.31)\end{array}$ & 33.16994 & 41.5043 \\
\hline & & $\begin{array}{l}\text { Primary } \\
\text { school }\end{array}$ & $\begin{array}{l}\text { 51.38(40.67, } \\
61.97)\end{array}$ & 19.29688 & 41.5043 \\
\hline & & $\begin{array}{l}\text { Secondary } \\
\text { school }+\end{array}$ & $\begin{array}{l}39.36(31.97 \\
47.27)\end{array}$ & 47.53319 & 41.5043 \\
\hline \multirow[t]{3}{*}{ Algeria } & \multirow[t]{3}{*}{2012} & No education & $\begin{array}{l}36.14(26.59 \\
46.93)\end{array}$ & 14.57784 & 41.73203 \\
\hline & & $\begin{array}{l}\text { Primary } \\
\text { school }\end{array}$ & $\begin{array}{l}46.84(38.73 \\
55.12)\end{array}$ & 20.97527 & 41.73203 \\
\hline & & $\begin{array}{l}\text { Secondary } \\
\text { school }+\end{array}$ & $\begin{array}{l}41.33(36.54 \\
46.28)\end{array}$ & 64.44688 & 41.73203 \\
\hline \multirow[t]{3}{*}{ Angola } & \multirow[t]{3}{*}{2015} & No education & $\begin{array}{l}29.85(25.05 \\
35.12)\end{array}$ & 27.03046 & 39.68827 \\
\hline & & $\begin{array}{l}\text { Primary } \\
\text { school }\end{array}$ & $\begin{array}{l}41.66(37.05 \\
46.42)\end{array}$ & 40.60347 & 39.68827 \\
\hline & & $\begin{array}{l}\text { Secondary } \\
\text { school + }\end{array}$ & $\begin{array}{l}45.42(40.04 \\
50.90)\end{array}$ & 32.36607 & 39.68827 \\
\hline \multirow[t]{3}{*}{ Benin } & \multirow[t]{3}{*}{2014} & No education & $\begin{array}{l}32.06(28.14 \\
36.25)\end{array}$ & 67.97112 & 32.54792 \\
\hline & & $\begin{array}{l}\text { Primary } \\
\text { school }\end{array}$ & $\begin{array}{l}31.01(23.75 \\
39.33)\end{array}$ & 23.47904 & 32.54792 \\
\hline & & $\begin{array}{l}\text { Secondary } \\
\text { school }+\end{array}$ & $\begin{array}{l}37.37(27.69 \\
48.18)\end{array}$ & 8.549847 & 32.54792 \\
\hline \multirow[t]{3}{*}{ Burundi } & \multirow[t]{3}{*}{2016} & No education & $\begin{array}{l}31.76(28.82 \\
34.85)\end{array}$ & 47.54028 & 34.95439 \\
\hline & & $\begin{array}{l}\text { Primary } \\
\text { school }\end{array}$ & $\begin{array}{l}36.98 \\
40.35)\end{array}$ & 44.59535 & 34.95439 \\
\hline & & $\begin{array}{l}\text { Secondary } \\
\text { school + }\end{array}$ & $\begin{array}{l}42.71(35.07 \\
50.72)\end{array}$ & 7.864367 & 34.95439 \\
\hline Cameroon & 2014 & No education & $\begin{array}{l}33.06(27.61 \\
39.00)\end{array}$ & 46.81973 & 39.29078 \\
\hline
\end{tabular}




\begin{tabular}{|c|c|c|c|c|c|}
\hline Country & Year & Subgroup & $\begin{array}{l}\text { Estimate (95\% } \\
\mathrm{Cl)}\end{array}$ & Population & $\begin{array}{l}\text { National } \\
\text { Coverage }\end{array}$ \\
\hline & & $\begin{array}{l}\text { Primary } \\
\text { school }\end{array}$ & $\begin{array}{l}39.02(34.23 \\
44.03)\end{array}$ & 33.56198 & 39.29078 \\
\hline & & $\begin{array}{l}\text { Secondary } \\
\text { school + }\end{array}$ & $\begin{array}{l}54.61(47.87 \\
61.18)\end{array}$ & 19.61829 & 39.29078 \\
\hline \multirow[t]{3}{*}{ Chad } & \multirow[t]{3}{*}{2014} & No education & $\begin{array}{l}25.66(23.05 \\
28.46)\end{array}$ & 59.05637 & 28.88636 \\
\hline & & $\begin{array}{l}\text { Primary } \\
\text { school }\end{array}$ & $\begin{array}{l}30.56(26.63 \\
34.80)\end{array}$ & 29.17677 & 28.88636 \\
\hline & & $\begin{array}{l}\text { Secondary } \\
\text { school + }\end{array}$ & $\begin{array}{l}40.86(34.63 \\
47.40)\end{array}$ & 11.76686 & 28.88636 \\
\hline \multirow[t]{3}{*}{ Comoros } & \multirow[t]{3}{*}{2012} & No education & $\begin{array}{l}48.65(39.93 \\
57.45)\end{array}$ & 46.24903 & 53.94333 \\
\hline & & $\begin{array}{l}\text { Primary } \\
\text { school }\end{array}$ & $\begin{array}{l}59.12(46.22 \\
70.87)\end{array}$ & 25.64578 & 53.94333 \\
\hline & & $\begin{array}{l}\text { Secondary } \\
\text { school + }\end{array}$ & $\begin{array}{l}57.65(46.86 \\
67.74)\end{array}$ & 28.10519 & 53.94333 \\
\hline \multirow[t]{3}{*}{ Congo } & \multirow[t]{3}{*}{2014} & No education & $\begin{array}{l}41.20(32.46 \\
50.52)\end{array}$ & 10.35354 & 42.24259 \\
\hline & & $\begin{array}{l}\text { Primary } \\
\text { school }\end{array}$ & $\begin{array}{l}41.20(35.89 \\
46.72)\end{array}$ & 33.93906 & 42.24259 \\
\hline & & $\begin{array}{l}\text { Secondary } \\
\text { school + }\end{array}$ & $\begin{array}{l}45.28(39.88 \\
50.79)\end{array}$ & 55.7074 & 42.24259 \\
\hline \multirow[t]{3}{*}{$\begin{array}{l}\text { Democratic Republic of the } \\
\text { Congo }\end{array}$} & \multirow[t]{3}{*}{2013} & No education & $\begin{array}{l}35.34(29.53 \\
41.61)\end{array}$ & 16.89113 & 39.94501 \\
\hline & & $\begin{array}{l}\text { Primary } \\
\text { school }\end{array}$ & $\begin{array}{l}42.00(37.65 \\
46.48)\end{array}$ & 44.65941 & 39.94501 \\
\hline & & $\begin{array}{l}\text { Secondary } \\
\text { school + }\end{array}$ & $\begin{array}{l}39.56(34.33 \\
45.05)\end{array}$ & 38.44947 & 39.94501 \\
\hline \multirow[t]{3}{*}{ Eswatini } & \multirow[t]{3}{*}{2014} & No education & $\begin{array}{l}55.55(37.17 \\
72.52)\end{array}$ & 6.741546 & 46.37177 \\
\hline & & $\begin{array}{l}\text { Primary } \\
\text { school }\end{array}$ & $\begin{array}{l}48.64(38.49 \\
58.89)\end{array}$ & 34.03322 & 46.37177 \\
\hline & & $\begin{array}{l}\text { Secondary } \\
\text { school + }\end{array}$ & $\begin{array}{l}44.02(36.12 \\
52.23)\end{array}$ & 59.22523 & 46.37177 \\
\hline Ethiopia & 2016 & No education & $\begin{array}{l}27.59(22.65 \\
33.15)\end{array}$ & 62.45697 & 28.65617 \\
\hline
\end{tabular}




\begin{tabular}{|c|c|c|c|c|c|}
\hline Country & Year & Subgroup & $\begin{array}{l}\text { Estimate (95\% } \\
\mathrm{Cl)}\end{array}$ & Population & $\begin{array}{l}\text { National } \\
\text { Coverage }\end{array}$ \\
\hline & & $\begin{array}{l}\text { Primary } \\
\text { school }\end{array}$ & $\begin{array}{l}31.87(24.98 \\
39.67)\end{array}$ & 30.10607 & 28.65617 \\
\hline & & $\begin{array}{l}\text { Secondary } \\
\text { school + }\end{array}$ & $\begin{array}{l}24.50(14.04 \\
39.18)\end{array}$ & 7.436958 & 28.65617 \\
\hline \multirow[t]{3}{*}{ Gabon } & 2012 & No education & $\begin{array}{l}31.17(13.14 \\
57.54)\end{array}$ & 4.16072 & 51.90965 \\
\hline & & $\begin{array}{l}\text { Primary } \\
\text { school }\end{array}$ & $\begin{array}{l}55.29(46.67 \\
63.60)\end{array}$ & 26.72619 & 51.90965 \\
\hline & & $\begin{array}{l}\text { Secondary } \\
\text { school + }\end{array}$ & $\begin{array}{l}51.84(44.29 \\
59.31)\end{array}$ & 69.11309 & 51.90965 \\
\hline \multirow[t]{3}{*}{ Gambia } & 2013 & No education & $\begin{array}{l}53.32(47.65 \\
58.90)\end{array}$ & 54.98156 & 50.84321 \\
\hline & & $\begin{array}{l}\text { Primary } \\
\text { school }\end{array}$ & $\begin{array}{l}46.38(36.93 \\
56.09)\end{array}$ & 17.09523 & 50.84321 \\
\hline & & $\begin{array}{l}\text { Secondary } \\
\text { school + }\end{array}$ & $\begin{array}{l}48.69(41.96 \\
55.46)\end{array}$ & 27.92321 & 50.84321 \\
\hline \multirow[t]{3}{*}{ Guinea } & 2016 & No education & $\begin{array}{l}40.77(36.45 \\
45.24)\end{array}$ & 70.64731 & 44.17994 \\
\hline & & $\begin{array}{l}\text { Primary } \\
\text { school }\end{array}$ & $\begin{array}{l}42.89(32.70 \\
53.72)\end{array}$ & 14.57406 & 44.17994 \\
\hline & & $\begin{array}{l}\text { Secondary } \\
\text { school + }\end{array}$ & $\begin{array}{l}61.70(51.71 \\
70.80)\end{array}$ & 14.77862 & 44.17994 \\
\hline \multirow[t]{3}{*}{ Guinea-Bissau } & 2014 & No education & $\begin{array}{l}49.70(43.11 \\
56.31)\end{array}$ & 51.7701 & 54.61463 \\
\hline & & $\begin{array}{l}\text { Primary } \\
\text { school }\end{array}$ & $\begin{array}{l}55.84(48.22 \\
63.20)\end{array}$ & 29.94337 & 54.61463 \\
\hline & & $\begin{array}{l}\text { Secondary } \\
\text { school + }\end{array}$ & $\begin{array}{l}66.48(55.89 \\
75.64)\end{array}$ & 18.28654 & 54.61463 \\
\hline \multirow[t]{3}{*}{ Kenya } & 2014 & No education & $\begin{array}{l}36.97(30.97 \\
43.40)\end{array}$ & 10.96732 & 45.1324 \\
\hline & & $\begin{array}{l}\text { Primary } \\
\text { school }\end{array}$ & $\begin{array}{l}42.92(39.51 \\
46.39)\end{array}$ & 60.10298 & 45.1324 \\
\hline & & $\begin{array}{l}\text { Secondary } \\
\text { school + }\end{array}$ & $\begin{array}{l}52.81(47.79 \\
57.77)\end{array}$ & 28.92971 & 45.1324 \\
\hline Lesotho & 2014 & No education & NA & 0.623956 & 61.38574 \\
\hline
\end{tabular}




\begin{tabular}{|c|c|c|c|c|c|}
\hline Country & Year & Subgroup & $\begin{array}{l}\text { Estimate (95\% } \\
\mathrm{Cl)}\end{array}$ & Population & $\begin{array}{l}\text { National } \\
\text { Coverage }\end{array}$ \\
\hline & & $\begin{array}{l}\text { Primary } \\
\text { school }\end{array}$ & $\begin{array}{l}52.40(43.47 \\
61.17)\end{array}$ & 51.47592 & 61.38574 \\
\hline & & $\begin{array}{l}\text { Secondary } \\
\text { school + }\end{array}$ & $\begin{array}{l}70.53(62.18 \\
77.70)\end{array}$ & 47.90012 & 61.38574 \\
\hline \multirow[t]{3}{*}{ Liberia } & \multirow[t]{3}{*}{2013} & No education & $\begin{array}{l}42.78(37.65 \\
48.06)\end{array}$ & 40.51356 & 46.43533 \\
\hline & & $\begin{array}{l}\text { Primary } \\
\text { school }\end{array}$ & $\begin{array}{l}49.59(43.60 \\
55.59)\end{array}$ & 32.20204 & 46.43533 \\
\hline & & $\begin{array}{l}\text { Secondary } \\
\text { school + }\end{array}$ & $\begin{array}{l}48.13(39.16 \\
57.21)\end{array}$ & 27.2844 & 46.43533 \\
\hline \multirow[t]{3}{*}{ Malawi } & \multirow[t]{3}{*}{2015} & No education & $\begin{array}{l}52.27(45.63 \\
58.82)\end{array}$ & 11.23334 & 56.68572 \\
\hline & & $\begin{array}{l}\text { Primary } \\
\text { school }\end{array}$ & $\begin{array}{l}56.45(53.74 \\
59.12)\end{array}$ & 68.98157 & 56.68572 \\
\hline & & $\begin{array}{l}\text { Secondary } \\
\text { school + }\end{array}$ & $\begin{array}{l}59.99(54.20 \\
65.50)\end{array}$ & 19.78509 & 56.68572 \\
\hline \multirow[t]{3}{*}{ Mali } & \multirow[t]{3}{*}{2015} & No education & $\begin{array}{l}26.34(23.72 \\
29.15)\end{array}$ & 81.46065 & 28.70292 \\
\hline & & $\begin{array}{l}\text { Primary } \\
\text { school }\end{array}$ & $\begin{array}{l}32.76(26.59 \\
39.60)\end{array}$ & 11.97664 & 28.70292 \\
\hline & & $\begin{array}{l}\text { Secondary } \\
\text { school + }\end{array}$ & $\begin{array}{l}37.38(28.73 \\
46.92)\end{array}$ & 6.562711 & 28.70292 \\
\hline \multirow[t]{3}{*}{ Mauritania } & \multirow[t]{3}{*}{2015} & No education & $\begin{array}{l}33.12(28.82 \\
37.71)\end{array}$ & 38.87771 & 34.50784 \\
\hline & & $\begin{array}{l}\text { Primary } \\
\text { school }\end{array}$ & $\begin{array}{l}32.84(27.45 \\
38.73)\end{array}$ & 24.21533 & 34.50784 \\
\hline & & $\begin{array}{l}\text { Secondary } \\
\text { school + }\end{array}$ & $\begin{array}{l}33.95(29.76 \\
38.41)\end{array}$ & 36.90696 & 34.50784 \\
\hline \multirow[t]{3}{*}{ Mozambique } & \multirow[t]{3}{*}{2015} & No education & $\begin{array}{l}30.85(20.74 \\
43.21)\end{array}$ & 24.80309 & 43.02846 \\
\hline & & $\begin{array}{l}\text { Primary } \\
\text { school }\end{array}$ & $\begin{array}{l}45.36(38.40 \\
52.50)\end{array}$ & 61.28754 & 43.02846 \\
\hline & & $\begin{array}{l}\text { Secondary } \\
\text { school + }\end{array}$ & $\begin{array}{l}54.43(42.79 \\
65.59)\end{array}$ & 13.90937 & 43.02846 \\
\hline Namibia & 2013 & No education & $\begin{array}{l}45.78(32.13 \\
60.10)\end{array}$ & 5.002066 & 51.98061 \\
\hline
\end{tabular}




\begin{tabular}{|c|c|c|c|c|c|}
\hline Country & Year & Subgroup & $\begin{array}{l}\text { Estimate (95\% } \\
\mathrm{Cl)}\end{array}$ & Population & $\begin{array}{l}\text { National } \\
\text { Coverage }\end{array}$ \\
\hline & & $\begin{array}{l}\text { Primary } \\
\text { school }\end{array}$ & $\begin{array}{l}51.86(44.97 \\
58.67)\end{array}$ & 30.06316 & 51.98061 \\
\hline & & $\begin{array}{l}\text { Secondary } \\
\text { school + }\end{array}$ & $\begin{array}{l}52.51(47.83 \\
57.14)\end{array}$ & 64.93478 & 51.98061 \\
\hline \multirow[t]{3}{*}{ Niger } & \multirow[t]{3}{*}{2012} & No education & $\begin{array}{l}43.45(39.40 \\
47.58)\end{array}$ & 83.6847 & 42.96736 \\
\hline & & $\begin{array}{l}\text { Primary } \\
\text { school }\end{array}$ & $\begin{array}{l}38.68(31.58 \\
46.30)\end{array}$ & 10.84407 & 42.96736 \\
\hline & & $\begin{array}{l}\text { Secondary } \\
\text { school + }\end{array}$ & $\begin{array}{l}44.83(35.18 \\
54.90)\end{array}$ & 5.471227 & 42.96736 \\
\hline \multirow[t]{3}{*}{ Nigeria } & \multirow[t]{3}{*}{2013} & No education & $\begin{array}{l}22.68(19.82 \\
25.83)\end{array}$ & 55.23032 & 29.23584 \\
\hline & & $\begin{array}{l}\text { Primary } \\
\text { school }\end{array}$ & $\begin{array}{l}34.05(29.57 \\
38.83)\end{array}$ & 18.63456 & 29.23584 \\
\hline & & $\begin{array}{l}\text { Secondary } \\
\text { school + }\end{array}$ & $\begin{array}{l}39.63(34.93 \\
44.54)\end{array}$ & 26.13512 & 29.23584 \\
\hline \multirow[t]{3}{*}{ Rwanda } & \multirow[t]{3}{*}{2014} & No education & $\begin{array}{l}21.28(14.59 \\
29.95)\end{array}$ & 16.7548 & 19.46708 \\
\hline & & $\begin{array}{l}\text { Primary } \\
\text { school }\end{array}$ & $\begin{array}{l}19.46(16.32 \\
23.03)\end{array}$ & 74.03515 & 19.46708 \\
\hline & & $\begin{array}{l}\text { Secondary } \\
\text { school + }\end{array}$ & $\begin{array}{l}16.19(9.45 \\
26.33)\end{array}$ & 9.210052 & 19.46708 \\
\hline \multirow[t]{3}{*}{ Sao Tome and Principe } & \multirow[t]{3}{*}{2014} & No education & NA & 5.513231 & 61.02909 \\
\hline & & $\begin{array}{l}\text { Primary } \\
\text { school }\end{array}$ & $\begin{array}{l}60.08(52.87 \\
66.88)\end{array}$ & 66.75923 & 61.02909 \\
\hline & & $\begin{array}{l}\text { Secondary } \\
\text { school + }\end{array}$ & $\begin{array}{l}61.02(47.16 \\
73.29)\end{array}$ & 27.72754 & 61.02909 \\
\hline \multirow[t]{3}{*}{ Senegal } & \multirow[t]{3}{*}{2014} & No education & $\begin{array}{l}51.46(46.36 \\
56.53)\end{array}$ & 65.45181 & 48.59681 \\
\hline & & $\begin{array}{l}\text { Primary } \\
\text { school }\end{array}$ & $\begin{array}{l}42.33(31.67 \\
53.74)\end{array}$ & 22.83674 & 48.59681 \\
\hline & & $\begin{array}{l}\text { Secondary } \\
\text { school + }\end{array}$ & $\begin{array}{l}44.78(32.10 \\
58.17)\end{array}$ & 11.71145 & 48.59681 \\
\hline Sierra Leone & 2013 & No education & $\begin{array}{l}58.23(53.76 \\
62.58)\end{array}$ & 69.36893 & 57.57013 \\
\hline
\end{tabular}




\begin{tabular}{|c|c|c|c|c|c|}
\hline Country & Year & Subgroup & $\begin{array}{l}\text { Estimate (95\% } \\
\mathrm{Cl)}\end{array}$ & Population & $\begin{array}{l}\text { National } \\
\text { Coverage }\end{array}$ \\
\hline & & $\begin{array}{l}\text { Primary } \\
\text { school }\end{array}$ & $\begin{array}{l}54.15(44.80 \\
63.22)\end{array}$ & 14.00004 & 57.57013 \\
\hline & & $\begin{array}{l}\text { Secondary } \\
\text { school + }\end{array}$ & $\begin{array}{l}57.65(49.48 \\
65.42)\end{array}$ & 16.63103 & 57.57013 \\
\hline \multirow[t]{3}{*}{ Togo } & \multirow[t]{3}{*}{2013} & No education & $\begin{array}{l}36.59(32.35 \\
41.05)\end{array}$ & 46.21846 & 33.42139 \\
\hline & & $\begin{array}{l}\text { Primary } \\
\text { school }\end{array}$ & $\begin{array}{l}29.58(24.16 \\
35.65)\end{array}$ & 35.01645 & 33.42139 \\
\hline & & $\begin{array}{l}\text { Secondary } \\
\text { school + }\end{array}$ & $\begin{array}{l}32.75(25.13 \\
41.41)\end{array}$ & 18.76509 & 33.42139 \\
\hline \multirow[t]{3}{*}{ Uganda } & \multirow[t]{3}{*}{2016} & No education & $\begin{array}{l}35.50(29.61 \\
41.87)\end{array}$ & 10.23754 & 37.38395 \\
\hline & & $\begin{array}{l}\text { Primary } \\
\text { school }\end{array}$ & $\begin{array}{l}36.58(34.07 \\
39.16)\end{array}$ & 62.6337 & 37.38395 \\
\hline & & $\begin{array}{l}\text { Secondary } \\
\text { school + }\end{array}$ & $\begin{array}{l}39.93(35.43 \\
44.61)\end{array}$ & 27.12877 & 37.38395 \\
\hline \multirow[t]{3}{*}{$\begin{array}{l}\text { United Republic of } \\
\text { Tanzania }\end{array}$} & \multirow[t]{3}{*}{2015} & No education & $\begin{array}{l}38.25(30.16 \\
47.05)\end{array}$ & 16.56646 & 45.45398 \\
\hline & & $\begin{array}{l}\text { Primary } \\
\text { school }\end{array}$ & $\begin{array}{l}45.66(40.78 \\
50.62)\end{array}$ & 65.43236 & 45.45398 \\
\hline & & $\begin{array}{l}\text { Secondary } \\
\text { school + }\end{array}$ & $\begin{array}{l}51.31(42.52 \\
60.02)\end{array}$ & 18.00118 & 45.45398 \\
\hline \multirow[t]{3}{*}{ Zambia } & \multirow[t]{3}{*}{2013} & No education & $\begin{array}{l}50.46(42.38 \\
58.53)\end{array}$ & 10.98515 & 56.17617 \\
\hline & & $\begin{array}{l}\text { Primary } \\
\text { school }\end{array}$ & $\begin{array}{l}56.74(53.02 \\
60.38)\end{array}$ & 55.00752 & 56.17617 \\
\hline & & $\begin{array}{l}\text { Secondary } \\
\text { school + }\end{array}$ & $\begin{array}{l}57.22(51.87 \\
62.41)\end{array}$ & 34.00733 & 56.17617 \\
\hline \multirow[t]{3}{*}{ Zimbabwe } & \multirow[t]{3}{*}{2014} & No education & $\begin{array}{l}48.90(34.94 \\
63.03)\end{array}$ & 3.129189 & 56.36424 \\
\hline & & $\begin{array}{l}\text { Primary } \\
\text { school }\end{array}$ & $\begin{array}{l}53.13(49.10 \\
57.12)\end{array}$ & 40.16053 & 56.36424 \\
\hline & & $\begin{array}{l}\text { Secondary } \\
\text { school + }\end{array}$ & $\begin{array}{l}59.06(55.51 \\
62.51)\end{array}$ & 56.71028 & 56.36424 \\
\hline
\end{tabular}

Among the 35 countries included in the study, in Sao Tome and Lesotho the results are not applicable as the estimate is missing for some groups. Of the 33 countries, 11 countries, namely Angola, Benin, 
Burundi, Cameron, Chad, Guinea, Guinea Bissau, Kenya, Mali, Mozambique and Nigeria had educational status inequality in ORT and continued feeding. However, the extent of the inequality fluctuated in different measures across countries. For instance, the average absolute educational inequality based on simple measure (D) was highest in Mozambique (23.6\%), Cameron (21.5\%) and Guinea (21\%). On the other hand, based on the complex measure (PAR), the average absolute inequality was highest in Guinea (17.5\%), Cameron (15.3\%) and Mozambique (11.4\%).The relative inequality was highest in Chad (41.5\%), Guinea (39.7\%) and Cameron (39\%) using the complex measure (PAF) (Table 4). 
Table 4

education-related inequality in ORT and continued feeding in the 35 low income, lower middle income and upper middle income SSA countries

\begin{tabular}{|c|c|c|c|c|c|}
\hline Country & Year & D & $\mathbf{R}$ & PAR & PAF \\
\hline Ghana & 2014 & $\begin{array}{l}0.53(-11.40 \\
12.48)\end{array}$ & $\begin{array}{l}1.01(0.70 \\
1.32)\end{array}$ & $0(-5.41,5.41)$ & $\begin{array}{l}0(-13.04 \\
13.04)\end{array}$ \\
\hline Algeria & 2012 & $\begin{array}{l}5.18(-6.19 \\
16.56)\end{array}$ & $\begin{array}{l}1.14(0.79, \\
1.49)\end{array}$ & $0(-6.20,6.20)$ & $\begin{array}{l}0(-14.86 \\
14.86)\end{array}$ \\
\hline Angola & 2015 & $\begin{array}{l}15.57(8.15 \\
22.98)\end{array}$ & $\begin{array}{l}1.52(1.20 \\
1.83)\end{array}$ & $\begin{array}{l}5.73(2.32 \\
9.14)\end{array}$ & $\begin{array}{l}14.44(5.85 \\
23.04)\end{array}$ \\
\hline Benin & 2014 & $\begin{array}{l}5.31(-5.81 \\
16.43)\end{array}$ & $\begin{array}{l}1.16(0.81 \\
1.52)\end{array}$ & $\begin{array}{l}5.10(3.29 \\
6.91)\end{array}$ & $\begin{array}{l}15.81(10.21 \\
21.42)\end{array}$ \\
\hline Burundi & 2016 & $\begin{array}{l}10.95(2.52 \\
19.37)\end{array}$ & $\begin{array}{l}1.34(1.06, \\
1.62)\end{array}$ & $\begin{array}{l}7.76(5.93, \\
9.58)\end{array}$ & $\begin{array}{l}22.20(16.97 \\
27.43)\end{array}$ \\
\hline Cameroon & 2014 & $\begin{array}{l}21.54(12.77 \\
30.31)\end{array}$ & $\begin{array}{l}1.65(1.30 \\
2.00)\end{array}$ & $\begin{array}{l}15.31(12.61 \\
18.02)\end{array}$ & $\begin{array}{l}38.98(32.10 \\
45.87)\end{array}$ \\
\hline Chad & 2014 & $\begin{array}{l}15.19(8.24 \\
22.14)\end{array}$ & $\begin{array}{l}1.59(1.29 \\
1.89)\end{array}$ & $\begin{array}{l}11.97(10.74 \\
13.20)\end{array}$ & $\begin{array}{l}41.45(37.18 \\
45.72)\end{array}$ \\
\hline Comoros & 2012 & $\begin{array}{l}8.99(-4.71 \\
22.70)\end{array}$ & $\begin{array}{l}1.18(0.88 \\
1.48)\end{array}$ & $\begin{array}{l}3.78(-0.89, \\
8.46)\end{array}$ & $\begin{array}{l}7.02(-1.66 \\
15.70)\end{array}$ \\
\hline Congo & 2014 & $\begin{array}{l}4.08(-6.53 \\
14.69)\end{array}$ & $\begin{array}{l}1.09(0.82, \\
1.37)\end{array}$ & $\begin{array}{l}1.80(-6.05 \\
9.67)\end{array}$ & $\begin{array}{l}4.15(-13.93 \\
22.25)\end{array}$ \\
\hline $\begin{array}{l}\text { Democratic Republic of } \\
\text { the Congo }\end{array}$ & 2013 & $\begin{array}{l}4.22(-3.85 \\
12.31)\end{array}$ & $\begin{array}{l}1.11(0.87 \\
1.36)\end{array}$ & $0(-3.92,3.92)$ & $\begin{array}{l}0 \text { (-9.81, } \\
9.813)\end{array}$ \\
\hline Eswatini & 2014 & $\begin{array}{l}-11.53(-31.58 \\
8.52)\end{array}$ & $\begin{array}{l}0.79(0.49 \\
1.09)\end{array}$ & $\begin{array}{l}0(-17.56 \\
17.56)\end{array}$ & $\begin{array}{l}0(-37.88 \\
37.88)\end{array}$ \\
\hline Ethiopia & 2016 & $\begin{array}{l}-3.09(-16.79 \\
10.59)\end{array}$ & $\begin{array}{l}0.88(0.39 \\
1.37)\end{array}$ & $0(-1.97,1.97)$ & $0(-6.89,6.89)$ \\
\hline Gabon & 2012 & $\begin{array}{l}20.67(-3.91 \\
45.27)\end{array}$ & $\begin{array}{l}1.66(0.39 \\
2.93)\end{array}$ & $\begin{array}{l}0(-16.49 \\
16.49)\end{array}$ & $\begin{array}{l}0(-31.76 \\
31.76)\end{array}$ \\
\hline Gambia & 2013 & $\begin{array}{l}-4.63(-13.41 \\
4.15)\end{array}$ & $\begin{array}{l}0.91(0.75 \\
1.07)\end{array}$ & $0(-2.44,2.44)$ & $0(-4.81,4.81)$ \\
\hline Guinea & 2016 & $\begin{array}{l}20.93(10.36 \\
31.50)\end{array}$ & $\begin{array}{l}1.51(1.22, \\
1.79)\end{array}$ & $\begin{array}{l}17.52(15.19 \\
19.86)\end{array}$ & $\begin{array}{l}39.67(34.38 \\
44.97)\end{array}$ \\
\hline Guinea-Bissau & 2014 & $\begin{array}{l}16.78(4.84 \\
28.72)\end{array}$ & $\begin{array}{l}1.33(1.06, \\
1.60)\end{array}$ & $\begin{array}{l}11.87(8.71 \\
15.03)\end{array}$ & $\begin{array}{l}21.74(15.95 \\
27.52)\end{array}$ \\
\hline Kenya & 2014 & $\begin{array}{l}15.83(7.83 \\
23.83)\end{array}$ & $\begin{array}{l}1.42(1.15, \\
1.70)\end{array}$ & $\begin{array}{l}7.67(2.58, \\
12.77)\end{array}$ & $\begin{array}{l}17.01(5.72, \\
28.30)\end{array}$ \\
\hline
\end{tabular}




\begin{tabular}{|c|c|c|c|c|c|}
\hline Country & Year & D & $\mathbf{R}$ & PAR & PAF \\
\hline Lesotho & 2014 & NA & NA & NA & NA \\
\hline Liberia & 2013 & $\begin{array}{l}5.34(-5.12 \\
15.81)\end{array}$ & $\begin{array}{l}1.12(0.87, \\
1.37)\end{array}$ & $\begin{array}{l}1.69(-1.55, \\
4.94)\end{array}$ & $\begin{array}{l}3.65(-3.34 \\
10.64)\end{array}$ \\
\hline Malawi & 2015 & $\begin{array}{l}7.71(-0.99 \\
16.43)\end{array}$ & $\begin{array}{l}1.14(0.96 \\
1.32)\end{array}$ & $\begin{array}{l}3.30(-1.29 \\
7.90)\end{array}$ & $\begin{array}{l}5.83(-2.28 \\
13.94)\end{array}$ \\
\hline Mali & 2015 & $\begin{array}{l}11.03(1.47 \\
20.60)\end{array}$ & $\begin{array}{l}1.41(1.04, \\
1.79)\end{array}$ & $\begin{array}{l}9.54(8.61 \\
10.47)\end{array}$ & $\begin{array}{l}34.28(30.95, \\
37.62)\end{array}$ \\
\hline Mauritania & 2015 & $\begin{array}{l}0.83(-5.35 \\
7.03)\end{array}$ & $\begin{array}{l}1.02(0.83 \\
1.21)\end{array}$ & $\begin{array}{l}0.59(-2.07 \\
3.25)\end{array}$ & $\begin{array}{l}1.78(-6.20 \\
9.76)\end{array}$ \\
\hline Mozambique & 2015 & $\begin{array}{l}23.57(7.41 \\
39.73)\end{array}$ & $\begin{array}{l}1.76(1.01 \\
2.51)\end{array}$ & $\begin{array}{l}11.40(4.67 \\
18.13)\end{array}$ & $\begin{array}{l}26.49(10.86, \\
42.13)\end{array}$ \\
\hline Namibia & 2013 & $\begin{array}{l}6.72(-8.32 \\
21.77)\end{array}$ & $\begin{array}{l}1.14(0.77 \\
1.51)\end{array}$ & $\begin{array}{l}0.53(-14.78 \\
15.85)\end{array}$ & $\begin{array}{l}1.02(-28.45 \\
30.49)\end{array}$ \\
\hline Niger & 2012 & $\begin{array}{l}1.38(-9.38 \\
12.15)\end{array}$ & $\begin{array}{l}1.03(0.78 \\
1.28)\end{array}$ & $\begin{array}{l}1.82(0.80 \\
2.85)\end{array}$ & $\begin{array}{l}4.24(1.86, \\
6.62)\end{array}$ \\
\hline Nigeria & 2013 & $\begin{array}{l}\text { 16.95(11.28, } \\
22.62)\end{array}$ & $\begin{array}{l}1.74(1.43 \\
2.06)\end{array}$ & $\begin{array}{l}10.40(8.91 \\
11.88)\end{array}$ & $\begin{array}{l}35.58(30.50 \\
40.66)\end{array}$ \\
\hline Rwanda & 2014 & $\begin{array}{l}-5.09(-16.39 \\
6.21)\end{array}$ & $\begin{array}{l}0.76(0.28 \\
1.23)\end{array}$ & $0(-5.89,5.89)$ & $\begin{array}{l}0(-30.27 \\
30.27)\end{array}$ \\
\hline Sao Tome and Principe & 2014 & NA & NA & NA & NA \\
\hline Senegal & 2014 & $\begin{array}{l}-6.68(-20.86 \\
7.49)\end{array}$ & $\begin{array}{l}0.87(0.59 \\
1.14)\end{array}$ & $0(-2.12,2.12)$ & $\begin{array}{l}0(-4.367 \\
4.36)\end{array}$ \\
\hline Sierra Leone & 2013 & $\begin{array}{l}-0.58(-9.71 \\
8.55)\end{array}$ & $\begin{array}{l}0.99(0.83 \\
1.14)\end{array}$ & $\begin{array}{l}0.08(-1.77 \\
1.95)\end{array}$ & $\begin{array}{l}0.15(-3.08 \\
3.38)\end{array}$ \\
\hline Togo & 2013 & $\begin{array}{l}-3.84(-13.08 \\
5.40)\end{array}$ & $\begin{array}{l}0.89(0.64 \\
1.14)\end{array}$ & $0(-3.26,3.26)$ & $0(-9.75,9.75)$ \\
\hline Uganda & 2016 & $\begin{array}{l}4.42(-3.24 \\
12.10)\end{array}$ & $\begin{array}{l}1.12(0.89, \\
1.35)\end{array}$ & $\begin{array}{l}2.55(-2.69 \\
7.79)\end{array}$ & $\begin{array}{l}6.82(-7.20 \\
20.85)\end{array}$ \\
\hline $\begin{array}{l}\text { United Republic of } \\
\text { Tanzania }\end{array}$ & 2015 & $\begin{array}{l}13.06(0.82 \\
25.30)\end{array}$ & $\begin{array}{l}1.34(0.96 \\
1.71)\end{array}$ & $\begin{array}{l}5.86(-0.58 \\
12.31)\end{array}$ & $\begin{array}{l}12.90(-1.29 \\
27.09)\end{array}$ \\
\hline Zambia & 2013 & $\begin{array}{l}6.75(-2.93 \\
16.45)\end{array}$ & $\begin{array}{l}1.13(0.92 \\
1.34)\end{array}$ & $\begin{array}{l}1.00(-5.19 \\
7.21)^{(}\end{array}$ & $\begin{array}{l}1.79(-9.24 \\
12.83)\end{array}$ \\
\hline Zimbabwe & 2014 & $\begin{array}{l}\text { 10.15(-4.66, } \\
24.97)\end{array}$ & $\begin{array}{l}1.20(0.84, \\
1.57)\end{array}$ & $\begin{array}{l}2.69(-11.43 \\
16.82)\end{array}$ & $\begin{array}{l}4.78(-20.28 \\
29.85)\end{array}$ \\
\hline
\end{tabular}


Without calculating summary measures, in $19(54 \%)$ of the countries, the average coverage of ORT and continued feeding is higher among male children than among female children (Table 5). According to the summary measures, however, we saw sex inequality in Niger, Kenya, Guinea-Bissau, and Senegal only (Table 6). 
Table 5

utilization of ORT and continued feeding for each subgroup of the sex stratifier across the 35 low income, lower middle income and upper middle income SSA countries

\begin{tabular}{|c|c|c|c|c|c|}
\hline Country & Year & Subgroup & $\begin{array}{l}\text { Estimate (95\% } \\
\mathrm{Cl})\end{array}$ & Population & $\begin{array}{l}\text { National } \\
\text { Coverage }\end{array}$ \\
\hline \multirow[t]{2}{*}{ Ghana } & \multirow[t]{2}{*}{2014} & Female & $\begin{array}{l}38.29(31.38 \\
45.72)\end{array}$ & 41.89582 & 41.5043 \\
\hline & & Male & $\begin{array}{l}43.81(37.73 \\
50.09)\end{array}$ & 58.10418 & 41.5043 \\
\hline \multirow[t]{2}{*}{ Algeria } & \multirow[t]{2}{*}{2012} & Female & $\begin{array}{l}42.43(37.00 \\
48.05)\end{array}$ & 47.28885 & 41.73203 \\
\hline & & Male & $\begin{array}{l}41.10(36.25 \\
46.13)\end{array}$ & 52.71115 & 41.73203 \\
\hline \multirow[t]{2}{*}{ Angola } & \multirow[t]{2}{*}{2015} & Female & $\begin{array}{l}39.54(34.82 \\
44.46)\end{array}$ & 46.72852 & 39.68827 \\
\hline & & Male & $\begin{array}{l}39.81(35.85 \\
43.91)\end{array}$ & 53.27148 & 39.68827 \\
\hline \multirow[t]{2}{*}{ Benin } & \multirow[t]{2}{*}{2014} & Female & $\begin{array}{l}30.18(26.02 \\
34.69)\end{array}$ & 50.82659 & 32.54792 \\
\hline & & Male & $\begin{array}{l}34.99(30.32 \\
39.97)\end{array}$ & 49.17341 & 32.54792 \\
\hline \multirow[t]{2}{*}{ Burundi } & \multirow[t]{2}{*}{2016} & Female & $\begin{array}{l}35.33(32.21 \\
38.58)\end{array}$ & 46.98909 & 34.95439 \\
\hline & & Male & $\begin{array}{l}34.62(31.80 \\
37.55)\end{array}$ & 53.01091 & 34.95439 \\
\hline \multirow[t]{2}{*}{ Cameroon } & \multirow[t]{2}{*}{2014} & Female & $\begin{array}{l}39.48(34.44 \\
44.75)\end{array}$ & 48.33535 & 39.29078 \\
\hline & & Male & $\begin{array}{l}39.11(35.13 \\
43.24)\end{array}$ & 51.66465 & 39.29078 \\
\hline \multirow[t]{2}{*}{ Chad } & \multirow[t]{2}{*}{2014} & Female & $\begin{array}{l}29.34(26.48 \\
32.38)\end{array}$ & 48.71667 & 28.88636 \\
\hline & & Male & $\begin{array}{l}28.44(25.47 \\
31.61)\end{array}$ & 51.28333 & 28.88636 \\
\hline \multirow[t]{2}{*}{ Comoros } & \multirow[t]{2}{*}{2012} & Female & $\begin{array}{l}57.89(49.42 \\
65.91)\end{array}$ & 48.73714 & 53.94333 \\
\hline & & Male & $\begin{array}{l}50.19(42.14 \\
58.22)\end{array}$ & 51.26286 & 53.94333 \\
\hline
\end{tabular}




\begin{tabular}{|c|c|c|c|c|c|}
\hline Country & Year & Subgroup & $\begin{array}{l}\text { Estimate (95\% } \\
\mathrm{Cl})\end{array}$ & Population & $\begin{array}{l}\text { National } \\
\text { Coverage }\end{array}$ \\
\hline \multirow[t]{2}{*}{ Congo } & \multirow[t]{2}{*}{2014} & Female & $\begin{array}{l}44.34(39.25 \\
49.54)\end{array}$ & 49.50343 & 42.24259 \\
\hline & & Male & $\begin{array}{l}40.18(35.26 \\
45.31)\end{array}$ & 50.49657 & 42.24259 \\
\hline \multirow[t]{2}{*}{$\begin{array}{l}\text { Democratic Republic of the } \\
\text { Congo }\end{array}$} & \multirow[t]{2}{*}{2013} & Female & $\begin{array}{l}39.73(35.62 \\
43.99)\end{array}$ & 47.28989 & 39.94501 \\
\hline & & Male & $\begin{array}{l}40.13(36.52 \\
43.84)\end{array}$ & 52.71011 & 39.94501 \\
\hline \multirow[t]{2}{*}{ Eswatini } & \multirow[t]{2}{*}{2014} & Female & $\begin{array}{l}48.29(40.86 \\
55.80)\end{array}$ & 45.53151 & 46.37177 \\
\hline & & Male & $\begin{array}{l}44.76(35.98, \\
53.88)\end{array}$ & 54.46849 & 46.37177 \\
\hline \multirow[t]{2}{*}{ Ethiopia } & \multirow[t]{2}{*}{2016} & Female & $\begin{array}{l}27.24(21.94 \\
33.27)\end{array}$ & 47.13117 & 28.65617 \\
\hline & & Male & $\begin{array}{l}29.91(24.71 \\
35.69)\end{array}$ & 52.86883 & 28.65617 \\
\hline \multirow[t]{2}{*}{ Gabon } & \multirow[t]{2}{*}{2012} & Female & $\begin{array}{l}50.31(42.51 \\
58.09)\end{array}$ & 48.83757 & 51.90965 \\
\hline & & Male & $\begin{array}{l}53.43(45.59 \\
61.10)\end{array}$ & 51.16243 & 51.90965 \\
\hline \multirow[t]{2}{*}{ Gambia } & \multirow[t]{2}{*}{2013} & Female & $\begin{array}{l}49.53(42.85 \\
56.21)\end{array}$ & 45.48225 & 50.84321 \\
\hline & & Male & $\begin{array}{l}51.93(46.68 \\
57.14)\end{array}$ & 54.51775 & 50.84321 \\
\hline \multirow[t]{2}{*}{ Guinea } & \multirow[t]{2}{*}{2016} & Female & $\begin{array}{l}44.22(38.37 \\
50.23)\end{array}$ & 45.44842 & 44.17994 \\
\hline & & Male & $\begin{array}{l}44.14(38.91 \\
49.50)\end{array}$ & 54.55158 & 44.17994 \\
\hline \multirow[t]{2}{*}{ Guinea-Bissau } & \multirow[t]{2}{*}{2014} & Female & $\begin{array}{l}48.42(42.19 \\
54.70)\end{array}$ & 45.15448 & 54.61463 \\
\hline & & Male & $\begin{array}{l}59.71(50.98 \\
67.86)\end{array}$ & 54.84552 & 54.61463 \\
\hline \multirow[t]{2}{*}{ Kenya } & \multirow[t]{2}{*}{2014} & Female & $\begin{array}{l}41.74(38.31 \\
45.26)\end{array}$ & 46.85591 & 45.1324 \\
\hline & & Male & $\begin{array}{l}48.11(44.70 \\
51.54)\end{array}$ & 53.14409 & 45.1324 \\
\hline
\end{tabular}




\begin{tabular}{|c|c|c|c|c|c|}
\hline Country & Year & Subgroup & $\begin{array}{l}\text { Estimate (95\% } \\
\mathrm{Cl)}\end{array}$ & Population & $\begin{array}{l}\text { National } \\
\text { Coverage }\end{array}$ \\
\hline \multirow[t]{2}{*}{ Lesotho } & \multirow[t]{2}{*}{2014} & Female & $\begin{array}{l}\text { 61.65(52.68, } \\
69.89)\end{array}$ & 49.22414 & 61.38574 \\
\hline & & Male & $\begin{array}{l}61.12(52.74, \\
68.88)\end{array}$ & 50.77586 & 61.38574 \\
\hline \multirow[t]{2}{*}{ Liberia } & \multirow[t]{2}{*}{2013} & Female & $\begin{array}{l}45.99(40.23 \\
51.86)\end{array}$ & 48.72026 & 46.43533 \\
\hline & & Male & $\begin{array}{l}46.85(41.83 \\
51.93)\end{array}$ & 51.27974 & 46.43533 \\
\hline \multirow[t]{2}{*}{ Malawi } & \multirow[t]{2}{*}{2015} & Female & $\begin{array}{l}55.00(51.47 \\
58.48)\end{array}$ & 46.4684 & 56.68572 \\
\hline & & Male & $\begin{array}{l}58.14(55.20 \\
61.03)\end{array}$ & 53.5316 & 56.68572 \\
\hline \multirow[t]{2}{*}{ Mali } & \multirow[t]{2}{*}{2015} & Female & $\begin{array}{l}29.36(25.80 \\
33.20)\end{array}$ & 46.17599 & 28.70292 \\
\hline & & Male & $\begin{array}{l}28.13(25.01 \\
31.47)\end{array}$ & 53.82401 & 28.70292 \\
\hline \multirow[t]{2}{*}{ Mauritania } & \multirow[t]{2}{*}{2015} & Female & $\begin{array}{l}31.83(28.07 \\
35.84)\end{array}$ & 48.99037 & 34.50784 \\
\hline & & Male & $\begin{array}{l}37.07 \text { (33.39, } \\
40.91)\end{array}$ & 51.00963 & 34.50784 \\
\hline \multirow[t]{2}{*}{ Mozambique } & \multirow[t]{2}{*}{2015} & Female & $\begin{array}{l}42.33(35.38 \\
49.61)\end{array}$ & 45.29924 & 43.02846 \\
\hline & & Male & $\begin{array}{l}43.59(35.67 \\
51.86)\end{array}$ & 54.70076 & 43.02846 \\
\hline \multirow[t]{2}{*}{ Namibia } & \multirow[t]{2}{*}{2013} & Female & $\begin{array}{l}49.94(44.32 \\
55.56)\end{array}$ & 47.2828 & 51.98061 \\
\hline & & Male & $\begin{array}{l}53.80(48.82 \\
58.70)\end{array}$ & 52.7172 & 51.98061 \\
\hline \multirow[t]{2}{*}{ Niger } & \multirow[t]{2}{*}{2012} & Female & $\begin{array}{l}45.26(40.86 \\
49.75)\end{array}$ & 47.37863 & 42.96736 \\
\hline & & Male & $\begin{array}{l}40.89 \text { (36.37, } \\
45.57)\end{array}$ & 52.62137 & 42.96736 \\
\hline \multirow[t]{2}{*}{ Nigeria } & \multirow[t]{2}{*}{2013} & Female & $\begin{array}{l}29.46(26.34 \\
32.79)\end{array}$ & 50.04135 & 29.23584 \\
\hline & & Male & $\begin{array}{l}29.00(26.08 \\
32.11)\end{array}$ & 49.95865 & 29.23584 \\
\hline
\end{tabular}




\begin{tabular}{|c|c|c|c|c|c|}
\hline Country & Year & Subgroup & $\begin{array}{l}\text { Estimate (95\% } \\
\mathrm{Cl)}\end{array}$ & Population & $\begin{array}{l}\text { National } \\
\text { Coverage }\end{array}$ \\
\hline \multirow[t]{2}{*}{ Rwanda } & \multirow[t]{2}{*}{2014} & Female & $\begin{array}{l}19.70(15.96 \\
24.07)\end{array}$ & 48.02026 & 19.46708 \\
\hline & & Male & $\begin{array}{l}\text { 19.24 (15.70, } \\
23.35)\end{array}$ & 51.97974 & 19.46708 \\
\hline \multirow[t]{2}{*}{ Sao Tome and Principe } & \multirow[t]{2}{*}{2014} & Female & $\begin{array}{l}55.48(46.79 \\
63.84)\end{array}$ & 38.32406 & 61.02909 \\
\hline & & Male & $\begin{array}{l}64.47(56.26 \\
71.91)\end{array}$ & 61.67594 & 61.02909 \\
\hline \multirow[t]{2}{*}{ Senegal } & \multirow[t]{2}{*}{2014} & Female & $\begin{array}{l}44.68(39.81 \\
49.66)\end{array}$ & 45.32852 & 48.59681 \\
\hline & & Male & $\begin{array}{l}51.83(46.20 \\
57.41)\end{array}$ & 54.67148 & 48.59681 \\
\hline \multirow[t]{2}{*}{ Sierra Leone } & \multirow[t]{2}{*}{2013} & Female & $\begin{array}{l}55.82(51.31 \\
60.23)\end{array}$ & 50.82598 & 57.57013 \\
\hline & & Male & $\begin{array}{l}59.37(54.24 \\
64.31)\end{array}$ & 49.17402 & 57.57013 \\
\hline \multirow[t]{2}{*}{ Togo } & \multirow[t]{2}{*}{2013} & Female & $\begin{array}{l}32.58(28.51 \\
36.93)\end{array}$ & 48.72497 & 33.42139 \\
\hline & & Male & $\begin{array}{l}34.21(29.88 \\
38.82)\end{array}$ & 51.27503 & 33.42139 \\
\hline \multirow[t]{2}{*}{ Uganda } & \multirow[t]{2}{*}{2016} & Female & $\begin{array}{l}35.51(32.38 \\
38.76)\end{array}$ & 46.24513 & 37.38395 \\
\hline & & Male & $\begin{array}{l}38.99(36.07 \\
42.00)\end{array}$ & 53.75487 & 37.38395 \\
\hline \multirow[t]{2}{*}{ United Republic of Tanzania } & \multirow[t]{2}{*}{2015} & Female & $\begin{array}{l}46.05(40.66 \\
51.53)\end{array}$ & 49.85485 & 45.45398 \\
\hline & & Male & $\begin{array}{l}44.85(39.41 \\
50.42)\end{array}$ & 50.14515 & 45.45398 \\
\hline \multirow[t]{2}{*}{ Zambia } & \multirow[t]{2}{*}{2013} & Female & $\begin{array}{l}56.47(52.43 \\
60.43)\end{array}$ & 47.86177 & 56.17617 \\
\hline & & Male & $\begin{array}{l}55.89(52.22 \\
59.50)\end{array}$ & 52.13823 & 56.17617 \\
\hline \multirow[t]{2}{*}{ Zimbabwe } & \multirow[t]{2}{*}{2014} & Female & $\begin{array}{l}56.68(52.88 \\
60.41)\end{array}$ & 50.24892 & 56.36424 \\
\hline & & Male & $\begin{array}{l}56.04(52.44 \\
59.57)\end{array}$ & 49.75108 & 56.36424 \\
\hline
\end{tabular}


Table 6

sex-related inequality in ORT and continued feeding in the 35 low income, lower middle income and upper middle income SSA countries

\begin{tabular}{|c|c|c|c|c|c|}
\hline \multirow[t]{2}{*}{ Country } & \multirow[t]{2}{*}{ Year } & \multicolumn{4}{|c|}{ Type and value of inequality measure with $95 \% \mathrm{Cl}$} \\
\hline & & D & $\mathbf{R}$ & PAR & PAF \\
\hline Ghana & 2014 & $\begin{array}{l}-5.51(-14.99 \\
3.95)\end{array}$ & $\begin{array}{l}0.87(0.66 \\
1.07)\end{array}$ & $\begin{array}{l}0(-3.24 \\
3.24)\end{array}$ & $0(-7.81,7.81)$ \\
\hline Algeria & 2012 & $\begin{array}{l}1.33(-6.08 \\
8.75)\end{array}$ & $\begin{array}{l}1.03(0.84 \\
1.21)\end{array}$ & $\begin{array}{l}0.70(-1.76 \\
3.17)\end{array}$ & $\begin{array}{l}1.68(-4.23 \\
7.59)\end{array}$ \\
\hline Angola & 2015 & $\begin{array}{l}-0.27(-6.55 \\
6.01)\end{array}$ & $\begin{array}{l}0.99(0.83 \\
1.15)\end{array}$ & $\begin{array}{l}0(-2.02, \\
2.02)\end{array}$ & $0(-5.09,5.09)$ \\
\hline Benin & 2014 & $\begin{array}{l}-4.81(-11.29 \\
1.67)\end{array}$ & $\begin{array}{l}0.86(0.69 \\
1.03)\end{array}$ & $\begin{array}{l}0(-2.66 \\
2.66)\end{array}$ & $0(-8.17,8.17)$ \\
\hline Burundi & 2016 & $\begin{array}{l}0.71(-3.57 \\
4.99)\end{array}$ & $\begin{array}{l}1.02(0.89 \\
1.14)\end{array}$ & $\begin{array}{l}0.37(-1.26 \\
2.01)\end{array}$ & $\begin{array}{l}1.07(-3.61 \\
5.77)\end{array}$ \\
\hline Cameroon & 2014 & $\begin{array}{l}0.37(-6.18 \\
6.92)\end{array}$ & $\begin{array}{l}1.00(0.84 \\
1.17)\end{array}$ & $\begin{array}{l}0.19(-2.27 \\
2.65)\end{array}$ & $\begin{array}{l}0.48(-5.77 \\
6.75)\end{array}$ \\
\hline Chad & 2014 & $\begin{array}{l}0.90(-3.34 \\
5.14)\end{array}$ & $\begin{array}{l}1.03(0.87 \\
1.18)\end{array}$ & $\begin{array}{l}0.46(-0.96 \\
1.88)\end{array}$ & $\begin{array}{l}1.60(-3.33 \\
6.53)\end{array}$ \\
\hline Comoros & 2012 & $\begin{array}{l}7.69(-3.84 \\
19.24)\end{array}$ & $\begin{array}{l}1.15(0.90 \\
1.40)\end{array}$ & $\begin{array}{l}3.94(-0.27 \\
8.16)\end{array}$ & $\begin{array}{l}7.31(-0.50 \\
15.13)\end{array}$ \\
\hline Congo & 2014 & $\begin{array}{l}4.15(-3.04 \\
11.35)\end{array}$ & $\begin{array}{l}1.10(0.91 \\
1.29)\end{array}$ & $\begin{array}{l}2.09(-0.30 \\
4.50)\end{array}$ & $\begin{array}{l}4.96(-0.72 \\
10.65)\end{array}$ \\
\hline $\begin{array}{l}\text { Democratic Republic of } \\
\text { the Congo }\end{array}$ & 2013 & $\begin{array}{l}-0.39(-5.95 \\
5.16)\end{array}$ & $\begin{array}{l}0.99(0.85 \\
1.12)\end{array}$ & $\begin{array}{l}0(-1.70 \\
1.70)\end{array}$ & $0(-4.26,4.26)$ \\
\hline Eswatini & 2014 & $\begin{array}{l}3.53(-8.16 \\
15.23)\end{array}$ & $\begin{array}{l}1.07(0.80 \\
1.35)\end{array}$ & $\begin{array}{l}1.92(-2.34 \\
6.19)\end{array}$ & $\begin{array}{l}4.15(-5.05 \\
13.35)\end{array}$ \\
\hline Ethiopia & 2016 & $\begin{array}{l}-2.67(-10.55 \\
5.20)\end{array}$ & $\begin{array}{l}0.91(0.65 \\
1.16)\end{array}$ & $\begin{array}{l}0(-2.38 \\
2.38)\end{array}$ & $0(-8.32,8.32)$ \\
\hline Gabon & 2012 & $\begin{array}{l}-3.12(-14.15 \\
7.90)\end{array}$ & $\begin{array}{l}0.94(0.74 \\
1.14)\end{array}$ & $\begin{array}{l}0(-3.45 \\
3.45)\end{array}$ & $0(-6.66,6.66)$ \\
\hline Gambia & 2013 & $\begin{array}{l}-2.40(-10.89 \\
6.07)\end{array}$ & $\begin{array}{l}0.95(0.79 \\
1.11)\end{array}$ & $\begin{array}{l}0(-2.46 \\
2.46)\end{array}$ & $0(-4.85,4.85)$ \\
\hline Guinea & 2016 & $\begin{array}{l}0.08(-7.86 \\
8.03)\end{array}$ & $\begin{array}{l}1.00(0.82 \\
1.18)\end{array}$ & $\begin{array}{l}0.04(-3.21 \\
3.30)\end{array}$ & $\begin{array}{l}0.10(-7.26 \\
7.46)\end{array}$ \\
\hline Guinea-Bissau & 2014 & $\begin{array}{l}-11.29(-21.83 \\
-0.74)\end{array}$ & $\begin{array}{l}0.81(0.65 \\
0.96)\end{array}$ & $\begin{array}{l}0(-2.99 \\
2.99)\end{array}$ & $0(-5.47,5.47)$ \\
\hline
\end{tabular}




\begin{tabular}{|c|c|c|c|c|c|}
\hline \multirow[t]{2}{*}{ Country } & \multirow[t]{2}{*}{ Year } & \multicolumn{4}{|c|}{ Type and value of inequality measure with $95 \% \mathrm{Cl}$} \\
\hline & & D & $\mathbf{R}$ & PAR & PAF \\
\hline Kenya & 2014 & $\begin{array}{l}-6.37(-11.25 \\
-1.49)\end{array}$ & $\begin{array}{l}0.86(0.77, \\
0.96)\end{array}$ & $\begin{array}{l}0(-1.72 \\
1.72)\end{array}$ & $0(-3.81,3.81)$ \\
\hline Lesotho & 2014 & $\begin{array}{l}0.53(-11.28 \\
12.35)\end{array}$ & $\begin{array}{l}1.00(0.81, \\
1.20)\end{array}$ & $\begin{array}{l}0.26(-4.81 \\
5.35)\end{array}$ & $\begin{array}{l}0.43(-7.84 \\
8.72)\end{array}$ \\
\hline Liberia & 2013 & $\begin{array}{l}-0.85(-8.55 \\
6.84)\end{array}$ & $\begin{array}{l}0.98(0.81, \\
1.14)\end{array}$ & $\begin{array}{l}0(-2.61, \\
2.61)\end{array}$ & $0(-5.62,5.62)$ \\
\hline Malawi & 2015 & $\begin{array}{l}-3.14(-7.70 \\
1.41)\end{array}$ & $\begin{array}{l}0.94(0.86 \\
1.02)\end{array}$ & $\begin{array}{l}0(-1.51 \\
1.51)\end{array}$ & $0(-2.67,2.67)$ \\
\hline Mali & 2015 & $\begin{array}{l}1.23(-3.67 \\
6.130\end{array}$ & $\begin{array}{l}1.04(0.86 \\
1.22)\end{array}$ & $\begin{array}{l}0.66(-1.02, \\
2.35)\end{array}$ & $\begin{array}{l}2.31(-3.57 \\
8.19)\end{array}$ \\
\hline Mauritania & 2015 & $\begin{array}{l}-5.24(-10.64 \\
0.15)\end{array}$ & $\begin{array}{l}0.85(0.72 \\
0.99)\end{array}$ & $\begin{array}{l}0(-2.00 \\
2.00)\end{array}$ & $0(-5.79,5.79)$ \\
\hline Mozambique & 2015 & $\begin{array}{l}-1.26(-12.06 \\
9.53)\end{array}$ & $\begin{array}{l}0.97(0.72, \\
1.21)\end{array}$ & $\begin{array}{l}0(-3.65, \\
3.65)\end{array}$ & $0(-8.48,8.48)$ \\
\hline Namibia & 2013 & $\begin{array}{l}-3.85(-11.33 \\
3.62)\end{array}$ & $\begin{array}{l}0.92(0.79 \\
1.06)\end{array}$ & $\begin{array}{l}0(-3.28 \\
3.28)\end{array}$ & $0(-6.31,6.31)$ \\
\hline Niger & 2012 & $\begin{array}{l}4.37(-2.01 \\
10.76)\end{array}$ & $\begin{array}{l}1.10(0.94, \\
1.27)\end{array}$ & $\begin{array}{l}2.30(0.08 \\
4.51)\end{array}$ & $\begin{array}{l}5.35(0.20 \\
10.51)\end{array}$ \\
\hline Nigeria & 2013 & $\begin{array}{l}0.46(-3.94 \\
4.87)\end{array}$ & $\begin{array}{l}1.01(0.86 \\
1.16)\end{array}$ & $\begin{array}{l}0.23(-1.40 \\
1.87)\end{array}$ & $\begin{array}{l}0.79(-4.81 \\
6.40)\end{array}$ \\
\hline Rwanda & 2014 & $\begin{array}{l}0.46(-5.08 \\
6.01)\end{array}$ & $\begin{array}{l}1.02(0.73, \\
1.31)\end{array}$ & $\begin{array}{l}0.24(-2.20 \\
2.69)\end{array}$ & $\begin{array}{l}1.24(-11.35 \\
13.83)\end{array}$ \\
\hline Sao Tome and Principe & 2014 & $\begin{array}{l}-8.99(-20.52 \\
2.5) 3\end{array}$ & $\begin{array}{l}0.86(0.69 \\
1.02)\end{array}$ & $\begin{array}{l}0(-4.04 \\
4.04)\end{array}$ & $0(-6.62,6.62)$ \\
\hline Senegal & 2014 & $\begin{array}{l}-7.14(-14.58 \\
0.28)\end{array}$ & $\begin{array}{l}0.86(0.72 \\
0.99)\end{array}$ & $\begin{array}{l}0(-2.65 \\
2.65)\end{array}$ & $0(-5.47,5.47)$ \\
\hline Sierra Leone & 2013 & $\begin{array}{l}-3.55(-10.27 \\
3.16)\end{array}$ & $\begin{array}{l}0.94(0.83, \\
1.04)\end{array}$ & $\begin{array}{l}0(-2.84 \\
2.84)\end{array}$ & $0(-4.94,4.94)$ \\
\hline Togo & 2013 & $\begin{array}{l}-1.62(-7.75 \\
4.49)\end{array}$ & $\begin{array}{l}0.95(0.77 \\
1.12)\end{array}$ & $\begin{array}{l}0(-2.93 \\
2.93)\end{array}$ & $0(-8.78,8.78)$ \\
\hline Uganda & 2016 & $\begin{array}{l}-3.48(-7.83, \\
0.86)\end{array}$ & $\begin{array}{l}0.91(0.80, \\
1.01)\end{array}$ & $\begin{array}{l}0(-1.65 \\
1.65)\end{array}$ & $0(-4.41,4.41)$ \\
\hline $\begin{array}{l}\text { United Republic of } \\
\text { Tanzania }\end{array}$ & 2015 & $\begin{array}{l}1.20(-6.54 \\
8.94)\end{array}$ & $\begin{array}{l}1.02(0.85 \\
1.20)\end{array}$ & $\begin{array}{l}0.60(-2.30 \\
3.50)\end{array}$ & $\begin{array}{l}1.32(-5.07 \\
7.72)\end{array}$ \\
\hline Zambia & 2013 & $\begin{array}{l}0.58(-4.83 \\
5.99)\end{array}$ & $\begin{array}{l}1.01(0.91, \\
1.10)\end{array}$ & $\begin{array}{l}0.30(-1.76 \\
2.37)\end{array}$ & $\begin{array}{l}0.53(-3.14 \\
4.22)\end{array}$ \\
\hline
\end{tabular}




\begin{tabular}{|llllll|}
\hline \multirow{2}{*}{ Country } & \multirow{2}{*}{ Year } & \multicolumn{4}{l|}{ Type and value of inequality measure with 95\% Cl } \\
\cline { 3 - 6 } & & D & R & PAR & PAF \\
\hline Zimbabwe & 2014 & $\begin{array}{l}0.64(-4.53, \\
\text { S.82) }\end{array}$ & $1.01(0.91$, & $0.32(-2.17$, & $0.56(-3.86$, \\
& & & $1.10)$ & $2.81)$ & $5.00)$ \\
\hline
\end{tabular}

\section{Residence Inequality}

The present study shows that out of the 35 countries, in twenty six of them, the average coverage of the ORT and continued feeding is higher among urban residents as compared to the rural residents. However, in Algeria, Burundi, Eswatini, Gabon, Sao Tome, Benin, Gambia, Senegal and Togo, at least the point estimate is higher among the rural than among urban residents (Table 7). 
Table 7

utilization of ORT and continued feeding for each subgroup of the residence tratifier across the 35 low income, lower middle income and upper middle income SSA countries

\begin{tabular}{|c|c|c|c|c|c|}
\hline Country & Year & Subgroup & $\begin{array}{l}\text { Estimate }(95 \% \\
\text { Cl) }\end{array}$ & Population & $\begin{array}{l}\text { National } \\
\text { Coverage }\end{array}$ \\
\hline \multirow[t]{2}{*}{ Ghana } & \multirow[t]{2}{*}{2014} & Rural & $\begin{array}{l}41.42(35.38 \\
47.72)\end{array}$ & 59.86536 & 41.5043 \\
\hline & & Urban & $\begin{array}{l}41.62(33.89 \\
49.78)\end{array}$ & 40.13464 & 41.5043 \\
\hline \multirow[t]{2}{*}{ Algeria } & \multirow[t]{2}{*}{2012} & Rural & $\begin{array}{l}43.40(36.70 \\
50.36)\end{array}$ & 41.2527 & 41.73203 \\
\hline & & Urban & $\begin{array}{l}40.55(35.82 \\
45.47)\end{array}$ & 58.7473 & 41.73203 \\
\hline \multirow[t]{2}{*}{ Angola } & \multirow[t]{2}{*}{2015} & Rural & $\begin{array}{l}31.10(27.25 \\
35.23)\end{array}$ & 37.04501 & 39.68827 \\
\hline & & Urban & $\begin{array}{l}44.73(40.38 \\
49.16)\end{array}$ & 62.95499 & 39.68827 \\
\hline \multirow[t]{2}{*}{ Benin } & \multirow[t]{2}{*}{2014} & Rural & $\begin{array}{l}32.63(28.26 \\
37.33)\end{array}$ & 56.76038 & 32.54792 \\
\hline & & Urban & $\begin{array}{l}32.43(27.32 \\
37.99)\end{array}$ & 43.23962 & 32.54792 \\
\hline \multirow[t]{2}{*}{ Burundi } & \multirow[t]{2}{*}{2016} & Rural & $\begin{array}{l}35.17(32.91 \\
37.50)\end{array}$ & 92.63887 & 34.95439 \\
\hline & & Urban & $\begin{array}{l}32.18(26.55 \\
38.39)\end{array}$ & 7.361135 & 34.95439 \\
\hline \multirow[t]{2}{*}{ Cameroon } & \multirow[t]{2}{*}{2014} & Rural & $\begin{array}{l}35.23(30.89 \\
39.82)\end{array}$ & 67.66472 & 39.29078 \\
\hline & & Urban & $\begin{array}{l}47.78(41.70 \\
53.92)\end{array}$ & 32.33528 & 39.29078 \\
\hline \multirow[t]{2}{*}{ Chad } & \multirow[t]{2}{*}{2014} & Rural & $\begin{array}{l}27.82(25.07 \\
30.75)\end{array}$ & 79.71847 & 28.88636 \\
\hline & & Urban & $\begin{array}{l}33.05(29.40 \\
36.91)\end{array}$ & 20.28153 & 28.88636 \\
\hline \multirow[t]{2}{*}{ Comoros } & \multirow[t]{2}{*}{2012} & Rural & $\begin{array}{l}50.08(42.35 \\
57.81)\end{array}$ & 73.52621 & 53.94333 \\
\hline & & Urban & $\begin{array}{l}64.66(51.87 \\
75.64)\end{array}$ & 26.47379 & 53.94333 \\
\hline
\end{tabular}




\begin{tabular}{|c|c|c|c|c|c|}
\hline Country & Year & Subgroup & $\begin{array}{l}\text { Estimate (95\% } \\
\mathrm{Cl})\end{array}$ & Population & $\begin{array}{l}\text { National } \\
\text { Coverage }\end{array}$ \\
\hline \multirow[t]{2}{*}{ Congo } & \multirow[t]{2}{*}{2014} & Rural & $\begin{array}{l}36.83(33.33 \\
40.48)\end{array}$ & 39.94055 & 42.24259 \\
\hline & & Urban & $\begin{array}{l}45.83(40.26 \\
51.51)\end{array}$ & 60.05945 & 42.24259 \\
\hline \multirow[t]{2}{*}{$\begin{array}{l}\text { Democratic Republic of the } \\
\text { Congo }\end{array}$} & \multirow[t]{2}{*}{2013} & Rural & $\begin{array}{l}37.40(33.51 \\
41.45)\end{array}$ & 65.95068 & 39.94501 \\
\hline & & Urban & $\begin{array}{l}44.87(40.15 \\
49.68)\end{array}$ & 34.04932 & 39.94501 \\
\hline \multirow[t]{2}{*}{ Eswatini } & \multirow[t]{2}{*}{2014} & Rural & $\begin{array}{l}47.57(41.72 \\
53.48)\end{array}$ & 77.92063 & 46.37177 \\
\hline & & Urban & $\begin{array}{l}42.13(26.36 \\
59.68)\end{array}$ & 22.07937 & 46.37177 \\
\hline \multirow[t]{2}{*}{ Ethiopia } & \multirow[t]{2}{*}{2016} & Rural & $\begin{array}{l}27.85(23.86 \\
32.23)\end{array}$ & 89.729 & 28.65617 \\
\hline & & Urban & $\begin{array}{l}35.64(20.82 \\
53.85)\end{array}$ & 10.271 & 28.65617 \\
\hline \multirow[t]{2}{*}{ Gabon } & \multirow[t]{2}{*}{2012} & Rural & $\begin{array}{l}56.12(50.33 \\
61.75)\end{array}$ & 16.91667 & 51.90965 \\
\hline & & Urban & $\begin{array}{l}51.05(43.99 \\
58.06)\end{array}$ & 83.08333 & 51.90965 \\
\hline \multirow[t]{2}{*}{ Gambia } & \multirow[t]{2}{*}{2013} & Rural & $\begin{array}{l}51.31(45.43 \\
57.16)\end{array}$ & 49.553 & 50.84321 \\
\hline & & Urban & $\begin{array}{l}50.37(43.16 \\
57.57)\end{array}$ & 50.447 & 50.84321 \\
\hline \multirow[t]{2}{*}{ Guinea } & \multirow[t]{2}{*}{2016} & Rural & $\begin{array}{l}39.44(34.98 \\
44.08)\end{array}$ & 68.96698 & 44.17994 \\
\hline & & Urban & $\begin{array}{l}54.70(47.69 \\
61.53)\end{array}$ & 31.03302 & 44.17994 \\
\hline \multirow[t]{2}{*}{ Guinea-Bissau } & \multirow[t]{2}{*}{2014} & Rural & $\begin{array}{l}50.03(45.21 \\
54.86)\end{array}$ & 56.07638 & 54.61463 \\
\hline & & Urban & $\begin{array}{l}60.45(51.59 \\
68.68)\end{array}$ & 43.92362 & 54.61463 \\
\hline \multirow[t]{2}{*}{ Kenya } & \multirow[t]{2}{*}{2014} & Rural & $\begin{array}{l}40.84(37.93 \\
43.81)\end{array}$ & 66.33608 & 45.1324 \\
\hline & & Urban & $\begin{array}{l}53.58(49.28 \\
57.83)\end{array}$ & 33.66392 & 45.1324 \\
\hline
\end{tabular}




\begin{tabular}{|c|c|c|c|c|c|}
\hline Country & Year & Subgroup & $\begin{array}{l}\text { Estimate (95\% } \\
\mathrm{Cl})\end{array}$ & Population & $\begin{array}{l}\text { National } \\
\text { Coverage }\end{array}$ \\
\hline \multirow[t]{2}{*}{ Lesotho } & \multirow[t]{2}{*}{2014} & Rural & $\begin{array}{l}57.90(51.35 \\
64.19)\end{array}$ & 75.28493 & 61.38574 \\
\hline & & Urban & $\begin{array}{l}71.98(56.75, \\
83.41)\end{array}$ & 24.71507 & 61.38574 \\
\hline \multirow[t]{2}{*}{ Liberia } & \multirow[t]{2}{*}{2013} & Rural & $\begin{array}{l}41.92(38.00 \\
45.94)\end{array}$ & 54.40137 & 46.43533 \\
\hline & & Urban & $\begin{array}{l}51.81(45.13 \\
58.44)\end{array}$ & 45.59863 & 46.43533 \\
\hline \multirow[t]{2}{*}{ Malawi } & \multirow[t]{2}{*}{2015} & Rural & $\begin{array}{l}56.01(53.70 \\
58.29)\end{array}$ & 84.28087 & 56.68572 \\
\hline & & Urban & $\begin{array}{l}60.30(51.92 \\
68.11)\end{array}$ & 15.71913 & 56.68572 \\
\hline \multirow[t]{2}{*}{ Mali } & \multirow[t]{2}{*}{2015} & Rural & $\begin{array}{l}27.17(24.36 \\
30.17)\end{array}$ & 82.89218 & 28.70292 \\
\hline & & Urban & $\begin{array}{l}36.11(30.74 \\
41.86)\end{array}$ & 17.10782 & 28.70292 \\
\hline \multirow[t]{2}{*}{ Mauritania } & \multirow[t]{2}{*}{2015} & Rural & $\begin{array}{l}33.15(29.86 \\
36.62)\end{array}$ & 65.70554 & 34.50784 \\
\hline & & Urban & $\begin{array}{l}37.09(31.92 \\
42.57)\end{array}$ & 34.29446 & 34.50784 \\
\hline \multirow[t]{2}{*}{ Mozambique } & \multirow[t]{2}{*}{2015} & Rural & $\begin{array}{l}40.86(34.04 \\
48.05)\end{array}$ & 76.48823 & 43.02846 \\
\hline & & Urban & $\begin{array}{l}50.07(42.60 \\
57.53)\end{array}$ & 23.51177 & 43.02846 \\
\hline \multirow[t]{2}{*}{ Namibia } & \multirow[t]{2}{*}{2013} & Rural & $\begin{array}{l}51.96(46.87 \\
57.02)\end{array}$ & 58.81007 & 51.98061 \\
\hline & & Urban & $\begin{array}{l}52.00(45.99 \\
57.95)\end{array}$ & 41.18993 & 51.98061 \\
\hline \multirow[t]{2}{*}{ Niger } & \multirow[t]{2}{*}{2012} & Rural & $\begin{array}{l}42.03(38.10 \\
46.07)\end{array}$ & 84.62476 & 42.96736 \\
\hline & & Urban & $\begin{array}{l}48.10(41.16 \\
55.11)\end{array}$ & 15.37524 & 42.96736 \\
\hline \multirow[t]{2}{*}{ Nigeria } & \multirow[t]{2}{*}{2013} & Rural & $\begin{array}{l}24.19(21.66 \\
26.92)\end{array}$ & 67.69464 & 29.23584 \\
\hline & & Urban & $\begin{array}{l}39.79(35.40 \\
44.36)\end{array}$ & 32.30536 & 29.23584 \\
\hline
\end{tabular}




\begin{tabular}{|c|c|c|c|c|c|}
\hline Country & Year & Subgroup & $\begin{array}{l}\text { Estimate (95\% } \\
\mathrm{Cl)}\end{array}$ & Population & $\begin{array}{l}\text { National } \\
\text { Coverage }\end{array}$ \\
\hline \multirow[t]{2}{*}{ Rwanda } & \multirow[t]{2}{*}{2014} & Rural & $\begin{array}{l}19.00(15.95 \\
22.47)\end{array}$ & 86.32555 & 19.46708 \\
\hline & & Urban & $\begin{array}{l}22.38(15.42, \\
31.32)\end{array}$ & 13.67445 & 19.46708 \\
\hline \multirow[t]{2}{*}{ Sao Tome and Principe } & \multirow[t]{2}{*}{2014} & Rural & $\begin{array}{l}62.59(50.66 \\
73.17)\end{array}$ & 30.55162 & 61.02909 \\
\hline & & Urban & $\begin{array}{l}60.33(52.80 \\
67.41)\end{array}$ & 69.44838 & 61.02909 \\
\hline \multirow[t]{2}{*}{ Senegal } & \multirow[t]{2}{*}{2014} & Rural & $\begin{array}{l}50.00(45.49 \\
54.52)\end{array}$ & 58.57504 & 48.59681 \\
\hline & & Urban & $\begin{array}{l}46.60(39.13 \\
54.22)\end{array}$ & 41.42496 & 48.59681 \\
\hline \multirow[t]{2}{*}{ Sierra Leone } & \multirow[t]{2}{*}{2013} & Rural & $\begin{array}{l}56.97(52.62, \\
61.22)\end{array}$ & 73.22478 & 57.57013 \\
\hline & & Urban & $\begin{array}{l}59.18(53.28 \\
64.83)\end{array}$ & 26.77522 & 57.57013 \\
\hline \multirow[t]{2}{*}{ Togo } & \multirow[t]{2}{*}{2013} & Rural & $\begin{array}{l}34.26(30.69 \\
38.02)\end{array}$ & 74.40346 & 33.42139 \\
\hline & & Urban & $\begin{array}{l}30.96(23.83 \\
39.13)\end{array}$ & 25.59654 & 33.42139 \\
\hline \multirow[t]{2}{*}{ Uganda } & \multirow[t]{2}{*}{2016} & Rural & $\begin{array}{l}36.06(33.69 \\
38.49)\end{array}$ & 81.43196 & 37.38395 \\
\hline & & Urban & $\begin{array}{l}43.17(37.87 \\
48.64)\end{array}$ & 18.56804 & 37.38395 \\
\hline \multirow[t]{2}{*}{ United Republic of Tanzania } & \multirow[t]{2}{*}{2015} & Rural & $\begin{array}{l}43.48(38.86 \\
48.22)\end{array}$ & 68.14753 & 45.45398 \\
\hline & & Urban & $\begin{array}{l}49.65(41.79 \\
57.53)\end{array}$ & 31.85247 & 45.45398 \\
\hline \multirow[t]{2}{*}{ Zambia } & \multirow[t]{2}{*}{2013} & Rural & $\begin{array}{l}54.29(50.72 \\
57.83)\end{array}$ & 61.96463 & 56.17617 \\
\hline & & Urban & $\begin{array}{l}59.23(53.95 \\
64.31)\end{array}$ & 38.03537 & 56.17617 \\
\hline \multirow[t]{2}{*}{ Zimbabwe } & \multirow[t]{2}{*}{2014} & Rural & $\begin{array}{l}55.62(52.56 \\
58.65)\end{array}$ & 76.30031 & 56.36424 \\
\hline & & Urban & $\begin{array}{l}58.72(53.35 \\
63.90)\end{array}$ & 23.69969 & 56.36424 \\
\hline
\end{tabular}


Table 8 shows sizable absolute and relative place of residence inequality in ORT and continued feeding in Angola, Cameron, Congo, Democratic Republic of Congo, Guinea, Kenya, Liberia, Mali, Nigeria and Uganda by the four measures. However, in Ethiopia, Lesotho, Mozambique, Niger, Malawi, Mauritania, Rwanda, Tanzania and Zambia the inequality was observed only by the complex measures. On the other hand, in 12 of the SSA countries: Ghana, Benin, Burundi, Algeria, Eswatini, Gabon, Gambia, Namibia, Sao Tome, Sierra Leone, Togo and Senegal, we did not record any urban-rural inequality. 
Table 8

residence-related inequality in ORT and continued feeding in the 35 low income, lower middle income and upper middle income SSA countries

\begin{tabular}{|c|c|c|c|c|c|}
\hline \multirow[t]{2}{*}{ Country } & \multirow[t]{2}{*}{ Year } & \multicolumn{4}{|c|}{ Type and value of inequality measure with $95 \% \mathrm{Cl}$} \\
\hline & & D & $\mathbf{R}$ & PAR & PAF \\
\hline Ghana & 2014 & $\begin{array}{l}0.20(-9.87 \\
10.28)\end{array}$ & $\begin{array}{l}1.00(0.76 \\
1.24)\end{array}$ & $\begin{array}{l}0.12(-3.01 \\
3.25)\end{array}$ & $\begin{array}{l}0.29(-7.25 \\
7.84)\end{array}$ \\
\hline Algeria & 2012 & $\begin{array}{l}-2.85(-11.24 \\
5.53)\end{array}$ & $\begin{array}{l}0.93(0.74 \\
1.11)\end{array}$ & $\begin{array}{l}0(-3.11 \\
3.11)\end{array}$ & $0(-7.46,7.46)$ \\
\hline Angola & 2015 & $\begin{array}{l}13.62(7.69, \\
19.55)\end{array}$ & $\begin{array}{l}1.43(1.20 \\
1.67)\end{array}$ & $\begin{array}{l}5.04(2.29 \\
7.80)\end{array}$ & $\begin{array}{l}12.72(5.77, \\
19.66)\end{array}$ \\
\hline Benin & 2014 & $\begin{array}{l}-0.20(-7.21 \\
6.79)\end{array}$ & $\begin{array}{l}0.99(0.77 \\
1.20)\end{array}$ & $\begin{array}{l}0(-2.28 \\
2.28)\end{array}$ & $0(-7.00,7.00)$ \\
\hline Burundi & 2016 & $\begin{array}{l}-2.98(-9.34 \\
3.36)\end{array}$ & $\begin{array}{l}0.91(0.73 \\
1.09)\end{array}$ & $\begin{array}{l}0(-0.48 \\
0.48)\end{array}$ & $0(-1.38,1.38)$ \\
\hline Cameroon & 2014 & $\begin{array}{l}12.55(4.98 \\
20.12)\end{array}$ & $\begin{array}{l}1.35(1.11, \\
1.60)\end{array}$ & $\begin{array}{l}8.49(6.69 \\
10.29)\end{array}$ & $\begin{array}{l}21.61(17.02 \\
26.20)\end{array}$ \\
\hline Chad & 2014 & $\begin{array}{l}5.22(0.52 \\
9.92)\end{array}$ & $\begin{array}{l}1.18(1.00 \\
1.36)\end{array}$ & $\begin{array}{l}4.16(3.40 \\
4.92)\end{array}$ & $\begin{array}{l}14.42(11.79 \\
17.05)\end{array}$ \\
\hline Comoros & 2012 & $\begin{array}{l}14.57(0.29 \\
28.86)\end{array}$ & $\begin{array}{l}1.29(0.97 \\
1.60)\end{array}$ & $\begin{array}{l}10.71(8.12 \\
13.31)\end{array}$ & $\begin{array}{l}19.87(15.06 \\
24.68)\end{array}$ \\
\hline Congo & 2014 & $\begin{array}{l}8.99(2.32 \\
15.66)\end{array}$ & $\begin{array}{l}1.24(1.04 \\
1.43)\end{array}$ & $\begin{array}{l}3.59(0.63 \\
6.55)\end{array}$ & $\begin{array}{l}8.50(1.50 \\
15.50)\end{array}$ \\
\hline $\begin{array}{l}\text { Democratic Republic of } \\
\text { the Congo }\end{array}$ & 2013 & $\begin{array}{l}7.47(1.27 \\
13.66)\end{array}$ & $\begin{array}{l}1.19(1.01 \\
1.37)\end{array}$ & $\begin{array}{l}4.92(3.61 \\
6.23)\end{array}$ & $\begin{array}{l}12.33(9.06 \\
15.60)\end{array}$ \\
\hline Eswatini & 2014 & $\begin{array}{l}-5.43(-23.61 \\
12.73)\end{array}$ & $\begin{array}{l}0.88(0.50 \\
1.26)\end{array}$ & $\begin{array}{l}0(-2.47, \\
2.47)\end{array}$ & $0(-5.33,5.33)$ \\
\hline Ethiopia & 2016 & $\begin{array}{l}7.79(-9.75 \\
25.33)\end{array}$ & $\begin{array}{l}1.27(0.63 \\
1.92)\end{array}$ & $\begin{array}{l}6.99(6.08, \\
7.90)\end{array}$ & $\begin{array}{l}24.39 \\
27.57)\end{array}$ \\
\hline Gabon & 2012 & $\begin{array}{l}-5.07(-14.14 \\
4.00)\end{array}$ & $\begin{array}{l}0.90(0.75 \\
1.06)\end{array}$ & $\begin{array}{l}0(-7.82 \\
7.82)\end{array}$ & $\begin{array}{l}0(-15.07 \\
15.07)\end{array}$ \\
\hline Gambia & 2013 & $\begin{array}{l}-0.93(-10.24 \\
8.36)\end{array}$ & $\begin{array}{l}0.98(0.80 \\
1.16)\end{array}$ & $\begin{array}{l}0(-2.72 \\
2.72)\end{array}$ & $0(-5.35,5.35)$ \\
\hline Guinea & 2016 & $\begin{array}{l}15.26(6.97 \\
23.55)\end{array}$ & $\begin{array}{l}1.38(1.14 \\
1.62)\end{array}$ & $\begin{array}{l}10.52(8.07 \\
12.97)\end{array}$ & $\begin{array}{l}23.82(18.28 \\
29.37)\end{array}$ \\
\hline Guinea-Bissau & 2014 & $\begin{array}{l}10.41(0.56 \\
20.26)\end{array}$ & $\begin{array}{l}1.20(1.00 \\
1.41)\end{array}$ & $\begin{array}{l}5.84(2.94 \\
8.73)\end{array}$ & $\begin{array}{l}10.69(5.39 \\
16.00)\end{array}$ \\
\hline
\end{tabular}




\begin{tabular}{|c|c|c|c|c|c|}
\hline \multirow[t]{2}{*}{ Country } & \multirow[t]{2}{*}{ Year } & \multicolumn{4}{|c|}{ Type and value of inequality measure with $95 \% \mathrm{Cl}$} \\
\hline & & D & $\mathbf{R}$ & PAR & PAF \\
\hline Kenya & 2014 & $\begin{array}{l}12.74(7.55 \\
17.94)\end{array}$ & $\begin{array}{l}1.31(1.17 \\
1.45)\end{array}$ & $\begin{array}{l}8.45(7.13 \\
9.78)\end{array}$ & $\begin{array}{l}18.73(15.80 \\
21.67)\end{array}$ \\
\hline Lesotho & 2014 & $\begin{array}{l}14.07(-0.82 \\
28.98)\end{array}$ & $\begin{array}{l}1.24(0.97, \\
1.51)\end{array}$ & $\begin{array}{l}10.59(7.69 \\
13.49)\end{array}$ & $\begin{array}{l}17.26(12.53 \\
21.99)\end{array}$ \\
\hline Liberia & 2013 & $\begin{array}{l}9.89(2.14 \\
17.65)\end{array}$ & $\begin{array}{l}1.23(1.03, \\
1.43)\end{array}$ & $\begin{array}{l}5.38(2.91 \\
7.85)\end{array}$ & $\begin{array}{l}11.59(6.28, \\
16.91)\end{array}$ \\
\hline Malawi & 2015 & $\begin{array}{l}4.29(-4.17 \\
12.76)\end{array}$ & $\begin{array}{l}1.07(0.92, \\
1.22)\end{array}$ & $\begin{array}{l}3.61(2.92, \\
4.31)\end{array}$ & $\begin{array}{l}6.38(5.15, \\
7.60)\end{array}$ \\
\hline Mali & 2015 & $\begin{array}{l}8.94(2.66, \\
15.22)\end{array}$ & $\begin{array}{l}1.32(1.07, \\
1.57)\end{array}$ & $\begin{array}{l}7.41(6.53, \\
8.29)\end{array}$ & $\begin{array}{l}25.83(22.77 \\
28.88)\end{array}$ \\
\hline Mauritania & 2015 & $\begin{array}{l}3.939-2.36 \\
10.23)\end{array}$ & $\begin{array}{l}1.11(0.92, \\
1.31)\end{array}$ & $\begin{array}{l}2.58(1.09 \\
4.07)\end{array}$ & $\begin{array}{l}7.48(3.17 \\
11.80)\end{array}$ \\
\hline Mozambique & 2015 & $\begin{array}{l}9.20(-1.03 \\
19.45)\end{array}$ & $\begin{array}{l}1.22(0.94, \\
1.50)\end{array}$ & $\begin{array}{l}7.04(4.77, \\
9.30)\end{array}$ & $\begin{array}{l}\text { 16.36 (11.10, } \\
21.62)\end{array}$ \\
\hline Namibia & 2013 & $\begin{array}{l}0.03(-7.81 \\
7.88)\end{array}$ & $\begin{array}{l}1.00(0.84 \\
1.15)\end{array}$ & $\begin{array}{l}0.01(-2.87 \\
2.91)\end{array}$ & $\begin{array}{l}0.03(-5.53 \\
5.61)\end{array}$ \\
\hline Niger & 2012 & $\begin{array}{l}6.06(-1.98 \\
14.12)\end{array}$ & $\begin{array}{l}1.14(0.94 \\
1.34)\end{array}$ & $\begin{array}{l}5.13(4.12 \\
6.14)\end{array}$ & $\begin{array}{l}11.95(9.60 \\
14.29)\end{array}$ \\
\hline Nigeria & 2013 & $\begin{array}{l}15.60(10.40 \\
20.79)\end{array}$ & $\begin{array}{l}1.64(1.38, \\
1.90)\end{array}$ & $\begin{array}{l}10.56(9.37 \\
11.75)\end{array}$ & $\begin{array}{l}36.12(32.06 \\
40.19)\end{array}$ \\
\hline Rwanda & 2014 & $\begin{array}{l}3.38(-5.18 \\
11.94)\end{array}$ & $\begin{array}{l}1.17(0.71, \\
1.64)\end{array}$ & $\begin{array}{l}2.91(1.85 \\
3.97)\end{array}$ & $\begin{array}{l}14.98(9.54 \\
20.43)\end{array}$ \\
\hline Sao Tome and Principe & 2014 & $\begin{array}{l}-2.25(-15.68 \\
11.17)\end{array}$ & $\begin{array}{l}0.96(0.75, \\
1.17)\end{array}$ & $\begin{array}{l}0(-7.59 \\
7.59)\end{array}$ & $\begin{array}{l}0(-12.43 \\
12.43)\end{array}$ \\
\hline Senegal & 2014 & $\begin{array}{l}-3.40(-12.19 \\
5.38)\end{array}$ & $\begin{array}{l}0.93(0.75, \\
1.10)\end{array}$ & $\begin{array}{l}0(-2.44, \\
2.44)\end{array}$ & $0(-5.04,5.04)$ \\
\hline Sierra Leone & 2013 & $\begin{array}{l}2.20(-4.98 \\
9.40)\end{array}$ & $\begin{array}{l}1.03(0.91, \\
1.16)\end{array}$ & $\begin{array}{l}1.61(-0.07 \\
3.30)\end{array}$ & $\begin{array}{l}2.80(-0.12 \\
5.73)\end{array}$ \\
\hline Togo & 2013 & $\begin{array}{l}-3.30(-11.78 \\
5.18)\end{array}$ & $\begin{array}{l}0.90(0.66 \\
1.14)\end{array}$ & $\begin{array}{l}0(-1.74 \\
1.74)\end{array}$ & $0(-5.21,5.21)$ \\
\hline Uganda & 2016 & $\begin{array}{l}7.11(1.21 \\
13.01)\end{array}$ & $\begin{array}{l}1.19(1.02, \\
1.36)\end{array}$ & $\begin{array}{l}5.79(4.92 \\
6.66)\end{array}$ & $\begin{array}{l}15.49(13.16 \\
17.83)\end{array}$ \\
\hline $\begin{array}{l}\text { United Republic of } \\
\text { Tanzania }\end{array}$ & 2015 & $\begin{array}{l}6.16(-3.02 \\
15.35)\end{array}$ & $\begin{array}{l}1.14(0.92 \\
1.36)\end{array}$ & $\begin{array}{l}4.20(2.19 \\
6.20)\end{array}$ & $\begin{array}{l}9.24(4.83 \\
13.65)\end{array}$ \\
\hline Zambia & 2013 & $\begin{array}{l}4.93(-1.35 \\
11.22)\end{array}$ & $\begin{array}{l}1.09(0.97, \\
1.21)\end{array}$ & $\begin{array}{l}3.05(1.37, \\
4.74)\end{array}$ & $\begin{array}{l}5.44(2.43, \\
8.45)\end{array}$ \\
\hline
\end{tabular}




\begin{tabular}{|llllll|}
\hline \multirow{2}{*}{ Country } & Year & \multicolumn{4}{l|}{ Type and value of inequality measure with $95 \% \mathrm{Cl}$} \\
\cline { 3 - 6 } & & D & R & PAR & PAF \\
\hline Zimbabwe & 2014 & $3.09(-2.99$, & $1.05(0.94$, & $2.36(0.98$, & $4.19(1.74$, \\
& & $9.19)$ & $1.16)$ & $3.74)$ & $6.64)$ \\
\hline
\end{tabular}

\section{Sub-national Regional Inequality}

Table 9 shows significant regional disparities in the utilization of ORT and continued feeding in the SSA countries. We recorded within country subnational regional disparities in the service in the studied countries, albeit the extent of disparities varied by country. With the exception of Comoros, Gabon, Rwanda, Sao Tome and Sierra Leone where no regional inequality was observed, other countries saw regional inequality with at least one measure. In Burundi, Cameron, Democratic Republic of Congo, Eswatini, Ethiopia, Guinea, Guinea Bissau, Kenya, Mali, Mauritania, Niger, Nigeria, Senegal, Togo, Uganda, Zambia and Zimbabwe, both absolute and relative regional inequality were demonstrated by all the four measures. The magnitude of regional inequality varied based on the measures of inequality. For instance, based on the PAF measure, the average regional disparity was highest in Ethiopia (105.2\%) followed by Ghana (80.1\%) and Mali (67.2\%). By the Difference measure, the highest were in Guinea Bissau (59.6\%) followed by Ghana (53\%) and Angola (51.3\%). See Table 10. 
Table 9

utilization of ORT and continued feeding for each subgroup of the sub-national region stratifier across the 35 low income, lower middle income and upper middle income SSA countries

\begin{tabular}{|c|c|c|c|c|c|}
\hline Country & Year & Subgroup & $\begin{array}{l}\text { Estimate } \\
(95 \% \mathrm{Cl})\end{array}$ & Population & $\begin{array}{l}\text { National } \\
\text { Coverage }\end{array}$ \\
\hline \multirow[t]{10}{*}{ Ghana } & \multirow[t]{10}{*}{2014} & 01 western & $\begin{array}{l}74.75 \\
(50.62, \\
89.53)\end{array}$ & 5.905278 & 41.5043 \\
\hline & & 02 central & $\begin{array}{l}56.50 \\
(39.56, \\
72.04)\end{array}$ & 7.995808 & 41.5043 \\
\hline & & 03 greater accra & $\begin{array}{l}33.27 \\
(16.76, \\
55.26)\end{array}$ & 9.86924 & 41.5043 \\
\hline & & 04 volta & $\begin{array}{l}21.67(8.16 \\
46.27)\end{array}$ & 4.500855 & 41.5043 \\
\hline & & 05 eastern & $\begin{array}{l}50.49 \\
(37.18 \\
63.73)\end{array}$ & 12.47621 & 41.5043 \\
\hline & & 06 ashanti & $\begin{array}{l}36.71 \\
(26.52, \\
48.23)\end{array}$ & 22.079 & 41.5043 \\
\hline & & 07 brong ahafo & $\begin{array}{l}25.93 \\
(19.35, \\
33.81)\end{array}$ & 12.84888 & 41.5043 \\
\hline & & 08 northern & $\begin{array}{l}38.47 \\
(25.91 \\
52.78)\end{array}$ & 16.79098 & 41.5043 \\
\hline & & 09 upper east & $\begin{array}{l}58.67 \\
(45.15 \\
71.00)\end{array}$ & 4.115501 & 41.5043 \\
\hline & & 10 upper west & $\begin{array}{l}49.70 \\
(38.70 \\
60.73)\end{array}$ & 3.418246 & 41.5043 \\
\hline \multirow[t]{3}{*}{ Algeria } & \multirow[t]{3}{*}{2012} & 01 EPT 1 : Nord-Centre & $\begin{array}{l}44.44 \\
(36.51 \\
52.66)\end{array}$ & 40.08263 & 41.73203 \\
\hline & & 02 EPT 2 : Nord-Est & $\begin{array}{l}51.43 \\
(40.67 \\
62.05)\end{array}$ & 8.174198 & 41.73203 \\
\hline & & 03 EPT 3 : Nord-Ouest & $\begin{array}{l}39.36 \\
(30.79 \\
48.64)\end{array}$ & 15.81673 & 41.73203 \\
\hline
\end{tabular}




\begin{tabular}{|c|c|c|c|c|c|}
\hline Country & Year & Subgroup & $\begin{array}{l}\text { Estimate } \\
(95 \% \mathrm{Cl})\end{array}$ & Population & $\begin{array}{l}\text { National } \\
\text { Coverage }\end{array}$ \\
\hline & & $\begin{array}{l}04 \text { EPT } 4 \text { : Hauts } \\
\text { Plateaux-Centre. }\end{array}$ & $\begin{array}{l}32.54 \\
(21.97 \\
45.26)\end{array}$ & 3.454743 & 41.73203 \\
\hline & & $\begin{array}{l}05 \text { EPT } 5 \text { : Hauts } \\
\text { Plateaux-Est }\end{array}$ & $\begin{array}{l}40.02 \\
(31.47 \\
49.23)\end{array}$ & 12.70428 & 41.73203 \\
\hline & & $\begin{array}{l}06 \text { EPT } 6 \text { : Hauts } \\
\text { Plateaux-Ouest }\end{array}$ & $\begin{array}{l}42.68 \\
(35.22 \\
50.49)\end{array}$ & 4.701748 & 41.73203 \\
\hline & & 07 EPT 7 : Sud & $\begin{array}{l}34.98 \\
(28.38 \\
42.21)\end{array}$ & 15.06566 & 41.73203 \\
\hline \multirow[t]{9}{*}{ Angola } & 2015 & 01 cabinda & $\begin{array}{l}37.99 \\
(23.26 \\
55.32)\end{array}$ & 1.429851 & 39.68827 \\
\hline & & 02 zaire & $\begin{array}{l}55.02 \\
(47.22 \\
62.59)\end{array}$ & 1.509453 & 39.68827 \\
\hline & & 03 uige & $\begin{array}{l}47.68 \\
(39.45 \\
56.04)\end{array}$ & 4.982247 & 39.68827 \\
\hline & & 04 luanda & $\begin{array}{l}47.58 \\
(40.56, \\
54.70)\end{array}$ & 29.63476 & 39.68827 \\
\hline & & 05 cuanza norte & $\begin{array}{l}53.96 \\
(43.911 \\
63.71)\end{array}$ & 1.283703 & 39.68827 \\
\hline & & 06 cuanza sul & $\begin{array}{l}26.43 \\
(19.21 \\
35.19)\end{array}$ & 11.06411 & 39.68827 \\
\hline & & 07 malanje & $\begin{array}{l}47.78 \\
(37.23 \\
58.52)\end{array}$ & 5.528303 & 39.68827 \\
\hline & & 08 lunda norte & $\begin{array}{l}40.53 \\
(31.96, \\
49.73)\end{array}$ & 3.243005 & 39.68827 \\
\hline & & 09 benguela & $\begin{array}{l}23.41 \\
(16.37 \\
32.30)\end{array}$ & 12.06692 & 39.68827 \\
\hline
\end{tabular}




\begin{tabular}{|c|c|c|c|c|c|}
\hline Country & Year & Subgroup & $\begin{array}{l}\text { Estimate } \\
(95 \% \mathrm{Cl})\end{array}$ & Population & $\begin{array}{l}\text { National } \\
\text { Coverage }\end{array}$ \\
\hline & & 10 huambo & $\begin{array}{l}40.31 \\
(30.10, \\
51.43)\end{array}$ & 7.782538 & 39.68827 \\
\hline & & 11 bie & $\begin{array}{l}29.84 \\
(19.63, \\
42.56)\end{array}$ & 4.009771 & 39.68827 \\
\hline & & 12 moxico & $\begin{array}{l}34.12 \\
(16.33 \\
57.90)\end{array}$ & 1.202983 & 39.68827 \\
\hline & & 13 cuando cubango & $\begin{array}{l}3.68(1.10 \\
11.59)\end{array}$ & 1.212588 & 39.68827 \\
\hline & & 14 namibe & $\begin{array}{l}39.71(31.3,0 \\
48.78)\end{array}$ & 1.640764 & 39.68827 \\
\hline & & 15 huila & $\begin{array}{l}46.42 \\
(35.0,9 \\
58.14)\end{array}$ & 9.021541 & 39.68827 \\
\hline & & 16 cunene & $\begin{array}{l}43.62 \\
(31.80, \\
56.20)\end{array}$ & 2.580286 & 39.68827 \\
\hline & & 17 lunda sul & $\begin{array}{l}35.11 \\
(24.77, \\
47.07)\end{array}$ & 1.384203 & 39.68827 \\
\hline & & 18 bengo & $\begin{array}{l}46.53 \\
(25.12 \\
69.29)\end{array}$ & 0.422983 & 39.68827 \\
\hline \multirow[t]{5}{*}{ Benin } & \multirow[t]{5}{*}{2014} & 01 Alibori & $\begin{array}{l}36.31 \\
(22.95 \\
52.17)\end{array}$ & 9.892942 & 32.54792 \\
\hline & & 02 Atacora & $\begin{array}{l}26.06 \\
(15.80 \\
39.84)\end{array}$ & 4.657463 & 32.54792 \\
\hline & & 03 Atlantique & $\begin{array}{l}27.84 \\
(17.93 \\
40.52)\end{array}$ & 14.64508 & 32.54792 \\
\hline & & 04 Borgou & $\begin{array}{l}37.82 \\
(28.70 \\
47.88)\end{array}$ & 14.08653 & 32.54792 \\
\hline & & 05 Collines & $\begin{array}{l}46.40 \\
(35.60 \\
57.55)\end{array}$ & 5.088295 & 32.54792 \\
\hline
\end{tabular}




\begin{tabular}{|c|c|c|c|c|c|}
\hline Country & Year & Subgroup & $\begin{array}{l}\text { Estimate } \\
(95 \% \mathrm{Cl})\end{array}$ & Population & $\begin{array}{l}\text { National } \\
\text { Coverage }\end{array}$ \\
\hline & & 06 Couffo & $\begin{array}{l}19.16 \\
(10.48 \\
32.42)\end{array}$ & 7.405164 & 32.54792 \\
\hline & & 07 Donga & $\begin{array}{l}42.41 \\
(30.91 \\
54.80)\end{array}$ & 6.001471 & 32.54792 \\
\hline & & 08 Littoral & $\begin{array}{l}36.65 \\
(29.09 \\
44.92)\end{array}$ & 4.549076 & 32.54792 \\
\hline & & 09 Mono & $\begin{array}{l}33.14 \\
(23.54 \\
44.37)\end{array}$ & 6.966779 & 32.54792 \\
\hline & & 10 OuAemAe & $\begin{array}{l}33.71 \\
(24.39 \\
44.50)\end{array}$ & 11.5029 & 32.54792 \\
\hline & & 11 Plateau & $\begin{array}{l}29.32 \\
(20.17 \\
40.51)\end{array}$ & 7.680445 & 32.54792 \\
\hline & & 12 Zou & $\begin{array}{l}25.29 \\
(16.70 \\
36.37)\end{array}$ & 7.523851 & 32.54792 \\
\hline \multirow[t]{6}{*}{ Burundi } & \multirow[t]{6}{*}{2016} & 01 bubanza & $\begin{array}{l}36.26 \\
(29.81 \\
43.24)\end{array}$ & 6.395139 & 34.95439 \\
\hline & & 02 bujumbura rural & $\begin{array}{l}38.31 \\
(28.35 \\
49.35)\end{array}$ & 4.80518 & 34.95439 \\
\hline & & 03 bururi & $\begin{array}{l}26.72 \\
(17.82 \\
38.01)\end{array}$ & 2.00979 & 34.95439 \\
\hline & & 04 cankuzo & $\begin{array}{l}48.05 \\
(35.73 \\
60.61)\end{array}$ & 1.784976 & 34.95439 \\
\hline & & 05 cibitoke & $\begin{array}{l}29.15 \\
(22.40 \\
36.96)\end{array}$ & 6.250945 & 34.95439 \\
\hline & & 06 gitega & $\begin{array}{l}35.03 \\
(27.23 \\
43.71)\end{array}$ & 8.799289 & 34.95439 \\
\hline
\end{tabular}




\begin{tabular}{|c|c|c|c|c|c|}
\hline Country & Year & Subgroup & $\begin{array}{l}\text { Estimate } \\
(95 \% \mathrm{Cl})\end{array}$ & Population & $\begin{array}{l}\text { National } \\
\text { Coverage }\end{array}$ \\
\hline & & 07 karusi & $\begin{array}{l}39.44 \\
(29.99, \\
49.75)\end{array}$ & 5.363564 & 34.95439 \\
\hline & & 08 kayanza & $\begin{array}{l}35.06 \\
(27.80 \\
43.09)\end{array}$ & 6.570189 & 34.95439 \\
\hline & & 09 kirundo & $\begin{array}{l}32.59 \\
(27.13 \\
38.56)\end{array}$ & 9.196535 & 34.95439 \\
\hline & & 10 makamba & $\begin{array}{l}37.76 \\
(26.52 \\
50.49)\end{array}$ & 5.076695 & 34.95439 \\
\hline & & 11 muramvya & $\begin{array}{l}39.28 \\
(29.27 \\
50.28)\end{array}$ & 3.300714 & 34.95439 \\
\hline & & 12 muyinga & $\begin{array}{l}42.78 \\
(35.85 \\
50.01)\end{array}$ & 10.94294 & 34.95439 \\
\hline & & 13 mwaro & $\begin{array}{l}15.74(8.98, \\
26.12)\end{array}$ & 2.599108 & 34.95439 \\
\hline & & 14 ngozi & $\begin{array}{l}26.82 \\
(20.20 \\
34.66)\end{array}$ & 8.990888 & 34.95439 \\
\hline & & 15 rutana & $\begin{array}{l}31.45 \\
(23.67 \\
40.44)\end{array}$ & 3.965691 & 34.95439 \\
\hline & & 16 ruyigi & $\begin{array}{l}37.23 \\
(26.55 \\
49.32)\end{array}$ & 3.92302 & 34.95439 \\
\hline & & 17 bujumbura mairie & $\begin{array}{l}30.60 \\
(21.70 \\
41.22)\end{array}$ & 3.814018 & 34.95439 \\
\hline & & 18 rumonge & $\begin{array}{l}40.01 \\
(30.38 \\
50.49)\end{array}$ & 6.211325 & 34.95439 \\
\hline \multirow[t]{2}{*}{ Cameroon } & 2014 & 01 Adamaoua & $\begin{array}{l}47.61 \\
(38.28, \\
57.11)\end{array}$ & 6.342471 & 39.29078 \\
\hline & & $\begin{array}{l}02 \text { Centre (sans } \\
\text { Yaounde) }\end{array}$ & $\begin{array}{l}40.13 \\
(29.65 \\
51.60)\end{array}$ & 6.22058 & 39.29078 \\
\hline
\end{tabular}




\begin{tabular}{|c|c|c|c|c|c|}
\hline Country & Year & Subgroup & $\begin{array}{l}\text { Estimate } \\
(95 \% \mathrm{Cl})\end{array}$ & Population & $\begin{array}{l}\text { National } \\
\text { Coverage }\end{array}$ \\
\hline & & 03 Douala & $\begin{array}{l}40.95 \\
(27.08, \\
56.43)\end{array}$ & 4.714651 & 39.29078 \\
\hline & & 04 Est & $\begin{array}{l}47.68 \\
(39.35 \\
56.15)\end{array}$ & 6.43828 & 39.29078 \\
\hline & & 05 Extreme-Nord & $\begin{array}{l}32.98 \\
(26.52, \\
40.16)\end{array}$ & 42.34943 & 39.29078 \\
\hline & & 06 Littoral (sans Douala) & $\begin{array}{l}62.80 \\
(42.56, \\
79.36)\end{array}$ & 1.778312 & 39.29078 \\
\hline & & 07 Nord & $\begin{array}{l}33.24 \\
(26.15, \\
41.17)\end{array}$ & 16.30826 & 39.29078 \\
\hline & & 08 Nord-Ouest & $\begin{array}{l}45.14 \\
(30.64, \\
60.52)\end{array}$ & 3.23699 & 39.29078 \\
\hline & & 09 Ouest & $\begin{array}{l}59.06 \\
(44.51, \\
72.17)\end{array}$ & 3.940744 & 39.29078 \\
\hline & & 10 Sud & $\begin{array}{l}50.26 \\
(36.20 \\
64.28)\end{array}$ & 1.940016 & 39.29078 \\
\hline & & 11 Sud-Ouest & $\begin{array}{l}41.91 \\
(21.98, \\
64.88)\end{array}$ & 3.099422 & 39.29078 \\
\hline & & 12 Yaounde & $\begin{array}{l}60.62 \\
(46.02, \\
73.54)\end{array}$ & 3.630837 & 39.29078 \\
\hline \multirow[t]{4}{*}{ Chad } & \multirow[t]{4}{*}{2014} & 01 batha & $\begin{array}{l}11.86(5.69, \\
23.08)\end{array}$ & 3.154713 & 28.88636 \\
\hline & & 02 borkou/tibesti & $\begin{array}{l}20.48(9.57 \\
38.52)\end{array}$ & 0.264882 & 28.88636 \\
\hline & & 03 chari baguirmi & $\begin{array}{l}19.51 \\
(14.04 \\
26.47)\end{array}$ & 4.599069 & 28.88636 \\
\hline & & 04 guera & $\begin{array}{l}34.02 \\
(25.10, \\
44.24)\end{array}$ & 3.506524 & 28.88636 \\
\hline
\end{tabular}




\begin{tabular}{|c|c|c|c|c|c|}
\hline Country & Year & Subgroup & $\begin{array}{l}\text { Estimate } \\
(95 \% \mathrm{Cl})\end{array}$ & Population & $\begin{array}{l}\text { National } \\
\text { Coverage }\end{array}$ \\
\hline & & 05 hadjer-lamis & $\begin{array}{l}32.99 \\
(23.62 \\
43.93)\end{array}$ & 7.300334 & 28.88636 \\
\hline & & 06 kanem & $\begin{array}{l}26.14 \\
(14.43 \\
42.62)\end{array}$ & 2.882109 & 28.88636 \\
\hline & & $07 \mathrm{lac}$ & $\begin{array}{l}28.64 \\
(18.31 \\
41.81)\end{array}$ & 3.708807 & 28.88636 \\
\hline & & 08 logone occidental & $\begin{array}{l}20.79 \\
(15.15 \\
27.84)\end{array}$ & 6.460039 & 28.88636 \\
\hline & & 09 logone oriental & $\begin{array}{l}27.00 \\
(20.53 \\
34.62)\end{array}$ & 14.1775 & 28.88636 \\
\hline & & 10 mandoul & $\begin{array}{l}30.63 \\
(21.50 \\
41.59)\end{array}$ & 8.386816 & 28.88636 \\
\hline & & 11 mayo kebbi est & $\begin{array}{l}36.34 \\
(24.43 \\
50.20)\end{array}$ & 7.960461 & 28.88636 \\
\hline & & 12 mayo kebbi ouest & $\begin{array}{l}26.10 \\
(19.42 \\
34.11)\end{array}$ & 5.900511 & 28.88636 \\
\hline & & 13 moyen chari & $\begin{array}{l}36.50 \\
(27.34 \\
46.75)\end{array}$ & 4.792608 & 28.88636 \\
\hline & & 14 ouaddai & $\begin{array}{l}22.52 \\
(14.47 \\
33.29)\end{array}$ & 4.50461 & 28.88636 \\
\hline & & 15 salamat & $\begin{array}{l}18.07 \\
(13.15 \\
24.30)\end{array}$ & 1.720352 & 28.88636 \\
\hline & & 16 tandjile & $\begin{array}{l}35.50 \\
(26.84 \\
45.23)\end{array}$ & 8.099291 & 28.88636 \\
\hline & & 17 wadi fira & $\begin{array}{l}37.36 \\
(26.51 \\
49.66)\end{array}$ & 0.829301 & 28.88636 \\
\hline
\end{tabular}




\begin{tabular}{|c|c|c|c|c|c|}
\hline Country & Year & Subgroup & $\begin{array}{l}\text { Estimate } \\
(95 \% \mathrm{Cl})\end{array}$ & Population & $\begin{array}{l}\text { National } \\
\text { Coverage }\end{array}$ \\
\hline & & 18 ndjamena & $\begin{array}{l}34.07 \\
(28.13 \\
40.54)\end{array}$ & 8.687822 & 28.88636 \\
\hline & & 19 barh el gazal & $\begin{array}{l}28.78 \\
(16.94, \\
44.46)\end{array}$ & 0.880659 & 28.88636 \\
\hline & & 20 ennedi & $\begin{array}{l}35.99 \\
(23.34 \\
50.94)\end{array}$ & 0.175428 & 28.88636 \\
\hline & & 21 sila & $\begin{array}{l}21.41 \\
(14.92 \\
29.74)\end{array}$ & 2.008161 & 28.88636 \\
\hline \multirow[t]{3}{*}{ Comoros } & 2012 & 01 Moheli & $\begin{array}{l}48.64 \\
(34.97 \\
62.51)\end{array}$ & 5.862743 & 53.94333 \\
\hline & & 02 Ndzouani & $\begin{array}{l}57.13 \\
(47.10 \\
66.60)\end{array}$ & 61.83141 & 53.94333 \\
\hline & & 03 Ngazidja & $\begin{array}{l}48.80 \\
(40.53 \\
57.13)\end{array}$ & 32.30585 & 53.94333 \\
\hline \multirow[t]{6}{*}{ Congo } & 2014 & 01 kouilou & $\begin{array}{l}48.81 \\
(39.70 \\
58.00)\end{array}$ & 3.065135 & 42.24259 \\
\hline & & 02 niari & $\begin{array}{l}46.10 \\
(38.12 \\
54.29)\end{array}$ & 7.223779 & 42.24259 \\
\hline & & 03 lekoumou & $\begin{array}{l}32.54 \\
(23.53, \\
43.05)\end{array}$ & 2.121613 & 42.24259 \\
\hline & & 04 bouenza & $\begin{array}{l}41.68 \\
(33.83 \\
49.97)\end{array}$ & 5.894389 & 42.24259 \\
\hline & & 05 pool & $\begin{array}{l}36.11 \\
(26.93 \\
46.43)\end{array}$ & 7.740024 & 42.24259 \\
\hline & & 06 plateaux & $\begin{array}{l}36.89 \\
(20.82 \\
56.50)\end{array}$ & 3.841696 & 42.24259 \\
\hline
\end{tabular}




\begin{tabular}{|c|c|c|c|c|c|}
\hline Country & Year & Subgroup & $\begin{array}{l}\text { Estimate } \\
(95 \% \mathrm{Cl})\end{array}$ & Population & $\begin{array}{l}\text { National } \\
\text { Coverage }\end{array}$ \\
\hline & & 07 cuvette & $\begin{array}{l}24.38 \\
(16.54 \\
34.39)\end{array}$ & 4.024014 & 42.24259 \\
\hline & & 08 CUVETTE OUEST & $\begin{array}{l}27.97 \\
(19.37 \\
38.57)\end{array}$ & 1.489775 & 42.24259 \\
\hline & & 09 sangha & $\begin{array}{l}41.41 \\
(32.92 \\
50.43)\end{array}$ & 2.559722 & 42.24259 \\
\hline & & 10 likouala & $\begin{array}{l}34.16 \\
(27.32, \\
41.73)\end{array}$ & 6.653182 & 42.24259 \\
\hline & & 11 brazzaville & $\begin{array}{l}45.85 \\
(38.59 \\
53.29)\end{array}$ & 41.69874 & 42.24259 \\
\hline & & 12 POINTE-NOIRE & $\begin{array}{l}45.32 \\
(35.89 \\
55.11)\end{array}$ & 13.68792 & 42.24259 \\
\hline \multirow[t]{7}{*}{$\begin{array}{l}\text { Democratic Republic } \\
\text { of the Congo }\end{array}$} & \multirow[t]{7}{*}{2013} & 01 Kinshasa & $\begin{array}{l}45.80 \\
(38.12 \\
53.67)\end{array}$ & 8.106149 & 39.94501 \\
\hline & & 02 Bandundu & $\begin{array}{l}44.03 \\
(34.79 \\
53.71)\end{array}$ & 13.23387 & 39.94501 \\
\hline & & 03 Bas-Congo & $\begin{array}{l}58.55 \\
(46.53 \\
69.63)\end{array}$ & 3.300011 & 39.94501 \\
\hline & & 04 Equateur & $\begin{array}{l}40.48 \\
(33.54 \\
47.84)\end{array}$ & 13.1954 & 39.94501 \\
\hline & & 05 Kasai-Occidental & $\begin{array}{l}35.82 \\
(30.34 \\
41.70)\end{array}$ & 10.68861 & 39.94501 \\
\hline & & 06 Kasai-Oriental & $\begin{array}{l}38.11 \\
(29.77 \\
47.23)\end{array}$ & 12.73778 & 39.94501 \\
\hline & & 07 Katanga & $\begin{array}{l}38.47 \\
(29.82 \\
47.91)\end{array}$ & 12.19382 & 39.94501 \\
\hline
\end{tabular}




\begin{tabular}{|c|c|c|c|c|c|}
\hline Country & Year & Subgroup & $\begin{array}{l}\text { Estimate } \\
(95 \% \mathrm{Cl})\end{array}$ & Population & $\begin{array}{l}\text { National } \\
\text { Coverage }\end{array}$ \\
\hline & & 08 Maniema & $\begin{array}{l}46.08 \\
(34.47 \\
58.13)\end{array}$ & 1.784687 & 39.94501 \\
\hline & & 09 Nord-Kivu & $\begin{array}{l}42.62 \\
(28.09 \\
58.54)\end{array}$ & 6.77575 & 39.94501 \\
\hline & & 10 Orientale & $\begin{array}{l}32.56 \\
(24.01 \\
42.45)\end{array}$ & 7.174815 & 39.94501 \\
\hline & & 11 Sud-Kivu & $\begin{array}{l}34.28 \\
(24.66, \\
45.39)\end{array}$ & 10.80911 & 39.94501 \\
\hline \multirow[t]{4}{*}{ Eswatini } & 2014 & 01 Hhohho & $\begin{array}{l}62.00 \\
(50.22, \\
72.51)\end{array}$ & 17.29594 & 46.37177 \\
\hline & & 02 Manzini & $\begin{array}{l}36.63 \\
(25.87 \\
48.90)\end{array}$ & 36.79971 & 46.37177 \\
\hline & & 03 Shiselweni & $\begin{array}{l}61.41 \\
(52.56 \\
69.56)\end{array}$ & 24.56155 & 46.37177 \\
\hline & & 04 Lubombo & $\begin{array}{l}33.19 \\
(23.55 \\
44.48)\end{array}$ & 21.3428 & 46.37177 \\
\hline \multirow[t]{5}{*}{ Ethiopia } & 2016 & 01 tigray & $\begin{array}{l}25.50 \\
(16.12 \\
37.87)\end{array}$ & 7.266876 & 28.65617 \\
\hline & & 02 afar & $\begin{array}{l}20.53 \\
(14.16 \\
28.80)\end{array}$ & 0.976671 & 28.65617 \\
\hline & & 03 amhara & $\begin{array}{l}26.84 \\
(18.18 \\
37.73)\end{array}$ & 21.99933 & 28.65617 \\
\hline & & 04 oromia & $\begin{array}{l}26.77 \\
(20.33 \\
34.36)\end{array}$ & 39.69011 & 28.65617 \\
\hline & & 05 somali & $\begin{array}{l}26.10 \\
(13.32, \\
44.79)\end{array}$ & 2.342253 & 28.65617 \\
\hline
\end{tabular}




\begin{tabular}{|c|c|c|c|c|c|}
\hline Country & Year & Subgroup & $\begin{array}{l}\text { Estimate } \\
(95 \% \mathrm{Cl})\end{array}$ & Population & $\begin{array}{l}\text { National } \\
\text { Coverage }\end{array}$ \\
\hline & & 06 benishangul & $\begin{array}{l}58.82 \\
(44.38 \\
71.88)\end{array}$ & 0.833683 & 28.65617 \\
\hline & & 07 snnpr & $\begin{array}{l}32.20 \\
(24.99 \\
40.37)\end{array}$ & 24.51446 & 28.65617 \\
\hline & & 08 gambela & $\begin{array}{l}25.94 \\
(17.88 \\
36.03)\end{array}$ & 0.299314 & 28.65617 \\
\hline & & 09 harari & $\begin{array}{l}37.15 \\
(24.59, \\
51.72)\end{array}$ & 0.212697 & 28.65617 \\
\hline & & 10 addis adaba & $\begin{array}{l}55.08 \\
(38.75 \\
70.39)\end{array}$ & 1.429125 & 28.65617 \\
\hline & & 11 dire dawa & $\begin{array}{l}29.75 \\
(20.35 \\
41.24)\end{array}$ & 0.435477 & 28.65617 \\
\hline \multirow[t]{7}{*}{ Gabon } & 2012 & 01 libreville-port-gentil & $\begin{array}{l}49.42 \\
(38.51 \\
60.38)\end{array}$ & 48.26995 & 51.90965 \\
\hline & & 02 estuaire & $\begin{array}{l}61.96 \\
(47.73 \\
74.40)\end{array}$ & 11.51206 & 51.90965 \\
\hline & & 03 haut-ogooue & $\begin{array}{l}56.47 \\
(43.21 \\
68.87)\end{array}$ & 12.21345 & 51.90965 \\
\hline & & 04 moyen-ogooue & $\begin{array}{l}49.68 \\
(36.49 \\
62.91)\end{array}$ & 3.405032 & 51.90965 \\
\hline & & 05 ngounie & $\begin{array}{l}58.45 \\
(46.15 \\
69.78)\end{array}$ & 5.169357 & 51.90965 \\
\hline & & 06 nyanga & $\begin{array}{l}63.58 \\
(52.26 \\
73.57)\end{array}$ & 3.212648 & 51.90965 \\
\hline & & 07 ogooue maritime & $\begin{array}{l}37.71 \\
(17.35 \\
63.57)\end{array}$ & 0.939501 & 51.90965 \\
\hline
\end{tabular}




\begin{tabular}{|c|c|c|c|c|c|}
\hline Country & Year & Subgroup & $\begin{array}{l}\text { Estimate } \\
(95 \% \mathrm{Cl})\end{array}$ & Population & $\begin{array}{l}\text { National } \\
\text { Coverage }\end{array}$ \\
\hline & & 08 ogooue-ivindo & $\begin{array}{l}45.62 \\
(35.07 \\
56.57)\end{array}$ & 6.027516 & 51.90965 \\
\hline & & 09 ogooue-lolo & $\begin{array}{l}41.39 \\
(30.94 \\
52.68)\end{array}$ & 4.087521 & 51.90965 \\
\hline & & 10 woleu-ntem & $\begin{array}{l}47.83 \\
(36.45 \\
59.44)\end{array}$ & 5.162971 & 51.90965 \\
\hline \multirow[t]{8}{*}{ Gambia } & \multirow[t]{8}{*}{2013} & 01 Banjul & $\begin{array}{l}41.73 \\
(33.16 \\
50.83)\end{array}$ & 2.386239 & 50.84321 \\
\hline & & 02 Kanifing & $\begin{array}{l}51.78 \\
(41.75, \\
61.67)\end{array}$ & 22.24152 & 50.84321 \\
\hline & & 03 Brikama & $\begin{array}{l}49.72 \\
(40.42 \\
59.04)\end{array}$ & 37.02072 & 50.84321 \\
\hline & & 04 Mansakonko & $\begin{array}{l}57.26 \\
(44.25 \\
69.34)\end{array}$ & 4.197206 & 50.84321 \\
\hline & & 05 Kerewan & $\begin{array}{l}53.97 \\
(45.73 \\
62.00)\end{array}$ & 8.70192 & 50.84321 \\
\hline & & 06 Kuntaur & $\begin{array}{l}52.23 \\
(38.27 \\
65.86)\end{array}$ & 7.923484 & 50.84321 \\
\hline & & 07 Janjanbureh & $\begin{array}{l}40.22 \\
(30.22 \\
51.10)\end{array}$ & 7.051451 & 50.84321 \\
\hline & & 08 Basse & $\begin{array}{l}55.77 \\
(44.25 \\
66.71)\end{array}$ & 10.47746 & 50.84321 \\
\hline \multirow[t]{2}{*}{ Guinea } & \multirow[t]{2}{*}{2016} & 01 BokAe & $\begin{array}{l}52.24 \\
(41.59, \\
62.70)\end{array}$ & 8.392693 & 44.17994 \\
\hline & & 02 Conakry & $\begin{array}{l}57.32 \\
(47.85, \\
66.28)\end{array}$ & 19.72138 & 44.17994 \\
\hline
\end{tabular}




\begin{tabular}{|c|c|c|c|c|c|}
\hline Country & Year & Subgroup & $\begin{array}{l}\text { Estimate } \\
(95 \% \mathrm{Cl})\end{array}$ & Population & $\begin{array}{l}\text { National } \\
\text { Coverage }\end{array}$ \\
\hline & & 03 Faranah & $\begin{array}{l}24.23 \\
(15.14 \\
36.42)\end{array}$ & 5.768013 & 44.17994 \\
\hline & & 04 Kankan & $\begin{array}{l}50.02 \\
(42.61 \\
57.42)\end{array}$ & 23.10604 & 44.17994 \\
\hline & & 05 Kindia & $\begin{array}{l}50.01 \\
(39.17 \\
60.84)\end{array}$ & 9.982872 & 44.17994 \\
\hline & & $06 \mathrm{LabAe}$ & $\begin{array}{l}13.36 \\
21.55)\end{array}$ & 15.50914 & 44.17994 \\
\hline & & 07 Mamou & $\begin{array}{l}43.12 \\
(34.19, \\
52.52)\end{array}$ & 11.88409 & 44.17994 \\
\hline & & 08 N'ZAerAekorAe & $\begin{array}{l}59.32 \\
(43.47 \\
73.44)\end{array}$ & 5.635769 & 44.17994 \\
\hline \multirow[t]{8}{*}{ Guinea-Bissau } & 2014 & 01 Tombali & $\begin{array}{l}75.13 \\
(62.52, \\
84.54)\end{array}$ & 6.575518 & 54.61463 \\
\hline & & 02 Quinara & $\begin{array}{l}46.11 \\
(34.46, \\
58.20)\end{array}$ & 4.109466 & 54.61463 \\
\hline & & 03 Oio & $\begin{array}{l}15.51(8.97 \\
25.47)\end{array}$ & 12.77767 & 54.61463 \\
\hline & & 04 Biombo & $\begin{array}{l}60.92 \\
(51.46, \\
69.63)\end{array}$ & 10.2329 & 54.61463 \\
\hline & & 05 Bolama/Bijagos & $\begin{array}{l}62.55 \\
(47.75, \\
75.33)\end{array}$ & 1.725259 & 54.61463 \\
\hline & & 06 Bafata & $\begin{array}{l}58.31 \\
(48.96 \\
67.09)\end{array}$ & 14.63102 & 54.61463 \\
\hline & & $07 \mathrm{Gabu}$ & $\begin{array}{l}49.56 \\
(38.77 \\
60.38)\end{array}$ & 9.249641 & 54.61463 \\
\hline & & 08 Cacheu & $\begin{array}{l}47.91 \\
(35.96 \\
60.10)\end{array}$ & 7.243852 & 54.61463 \\
\hline
\end{tabular}




\begin{tabular}{|c|c|c|c|c|c|}
\hline Country & Year & Subgroup & $\begin{array}{l}\text { Estimate } \\
(95 \% \mathrm{Cl})\end{array}$ & Population & $\begin{array}{l}\text { National } \\
\text { Coverage }\end{array}$ \\
\hline & & $09 \mathrm{sab}$ & $\begin{array}{l}65.45 \\
(55.33 \\
74.34)\end{array}$ & 33.45468 & 54.61463 \\
\hline \multirow[t]{8}{*}{ Kenya } & \multirow[t]{8}{*}{2014} & 01 coast & $\begin{array}{l}42.11 \\
(36.52, \\
47.92)\end{array}$ & 12.00712 & 45.1324 \\
\hline & & 02 north eastern & $\begin{array}{l}31.42 \\
(22.11 \\
42.51)\end{array}$ & 1.706665 & 45.1324 \\
\hline & & 03 eastern & $\begin{array}{l}43.94 \\
(37.35 \\
50.75)\end{array}$ & 11.25218 & 45.1324 \\
\hline & & 04 central & $\begin{array}{l}40.49 \\
(32.44 \\
49.08)\end{array}$ & 6.337812 & 45.1324 \\
\hline & & 05 rift valley & $\begin{array}{l}48.16 \\
(43.96 \\
52.38)\end{array}$ & 25.24882 & 45.1324 \\
\hline & & 06 western & $\begin{array}{l}33.94 \\
(27.33, \\
41.24)\end{array}$ & 15.33321 & 45.1324 \\
\hline & & 07 nyanza & $\begin{array}{l}40.04 \\
(34.87 \\
45.44)\end{array}$ & 17.57834 & 45.1324 \\
\hline & & 08 nairobi & $\begin{array}{l}72.35 \\
(62.49 \\
80.43)\end{array}$ & 10.53585 & 45.1324 \\
\hline \multirow[t]{5}{*}{ Lesotho } & \multirow[t]{5}{*}{2014} & 01 botha-bothe & NA & 4.965781 & 61.38574 \\
\hline & & 02 leribe & $\begin{array}{l}62.46 \\
(49.25 \\
74.04)\end{array}$ & 20.28227 & 61.38574 \\
\hline & & 03 berea & $\begin{array}{l}\text { 59.15(44.17, } \\
72.60)\end{array}$ & 11.10362 & 61.38574 \\
\hline & & 04 maseru & $\begin{array}{l}70.18 \\
(53.94 \\
82.54)\end{array}$ & 28.21801 & 61.38574 \\
\hline & & 05 mafeteng & $\begin{array}{l}56.60 \\
(38.61, \\
73.00)\end{array}$ & 8.135024 & 61.38574 \\
\hline
\end{tabular}




\begin{tabular}{|c|c|c|c|c|c|}
\hline Country & Year & Subgroup & $\begin{array}{l}\text { Estimate } \\
(95 \% \mathrm{Cl})\end{array}$ & Population & $\begin{array}{l}\text { National } \\
\text { Coverage }\end{array}$ \\
\hline & & 06 mohales hoek & $\begin{array}{l}55.06 \\
(29.65 \\
78.07)\end{array}$ & 7.991718 & 61.38574 \\
\hline & & 07 quthing & NA & 3.401176 & 61.38574 \\
\hline & & 08 qachas-nek & NA & 2.202532 & 61.38574 \\
\hline & & 09 mokhotlong & $\begin{array}{l}35.97 \\
(23.32, \\
50.92)\end{array}$ & 8.757536 & 61.38574 \\
\hline & & 10 thaba tseka & NA & 4.942333 & 61.38574 \\
\hline \multirow[t]{5}{*}{ Liberia } & \multirow[t]{5}{*}{2013} & 01 North Western & $\begin{array}{l}52.96 \\
(45.59 \\
60.21)\end{array}$ & 8.503247 & 46.43533 \\
\hline & & 02 South Central & $\begin{array}{l}50.73 \\
(43.05 \\
58.38)\end{array}$ & 39.72573 & 46.43533 \\
\hline & & 03 South Eastern A & $\begin{array}{l}38.94 \\
(34.02, \\
44.09)\end{array}$ & 9.555145 & 46.43533 \\
\hline & & 04 South Eastern B & $\begin{array}{l}46.31 \\
(36.94 \\
55.94)\end{array}$ & 9.69338 & 46.43533 \\
\hline & & 05 North Central & $\begin{array}{l}41.71 \\
(36.14 \\
47.49)\end{array}$ & 32.5225 & 46.43533 \\
\hline \multirow[t]{3}{*}{ Malawi } & \multirow[t]{3}{*}{2015} & 01 northern region & $\begin{array}{l}43.39 \\
(35.74 \\
51.36)\end{array}$ & 9.453849 & 56.68572 \\
\hline & & 02 central region & $\begin{array}{l}57.97 \\
(54.27 \\
61.59)\end{array}$ & 46.96595 & 56.68572 \\
\hline & & 03 southern region & $\begin{array}{l}58.17 \\
(55.12, \\
61.16)\end{array}$ & 43.5802 & 56.68572 \\
\hline \multirow[t]{2}{*}{ Mali } & \multirow[t]{2}{*}{2015} & 01 Kayes & $\begin{array}{l}31.25 \\
(26.67 \\
36.22)\end{array}$ & 20.23304 & 28.70292 \\
\hline & & 02 Koulikoro & $\begin{array}{l}32.91 \\
(25.89, \\
40.78)\end{array}$ & 14.34522 & 28.70292 \\
\hline
\end{tabular}




\begin{tabular}{|c|c|c|c|c|c|}
\hline Country & Year & Subgroup & $\begin{array}{l}\text { Estimate } \\
(95 \% \mathrm{Cl})\end{array}$ & Population & $\begin{array}{l}\text { National } \\
\text { Coverage }\end{array}$ \\
\hline & & 03 Sikasso & $\begin{array}{l}25.14 \\
(18.52, \\
33.17)\end{array}$ & 13.00799 & 28.70292 \\
\hline & & 04 Segou & $\begin{array}{l}19.15 \\
(14.56 \\
24.77)\end{array}$ & 26.42864 & 28.70292 \\
\hline & & 05 Mopti & $\begin{array}{l}31.94 \\
(24.26 \\
40.74)\end{array}$ & 9.741893 & 28.70292 \\
\hline & & 06 Tombouctou & $\begin{array}{l}25.28 \\
(18.03 \\
34.22)\end{array}$ & 5.601547 & 28.70292 \\
\hline & & 07 Gao & $\begin{array}{l}33.17 \\
(24.37 \\
43.33)\end{array}$ & 2.10544 & 28.70292 \\
\hline & & 08 Bamako & $\begin{array}{l}48.00 \\
(40.95 \\
55.14)\end{array}$ & 8.536235 & 28.70292 \\
\hline \multirow[t]{7}{*}{ Mauritania } & 2015 & 01 Hodh charghy & $\begin{array}{l}23.50 \\
(15.68 \\
33.68)\end{array}$ & 7.60384 & 34.50784 \\
\hline & & 02 Hodh Gharby & $\begin{array}{l}32.76 \\
(25.10 \\
41.45)\end{array}$ & 10.46105 & 34.50784 \\
\hline & & 03 Assaba & $\begin{array}{l}34.44 \\
(25.48 \\
44.65)\end{array}$ & 10.54789 & 34.50784 \\
\hline & & 04 Gorgol & $\begin{array}{l}31.37 \\
(25.90 \\
37.40)\end{array}$ & 20.82206 & 34.50784 \\
\hline & & 05 Brakna & $\begin{array}{l}27.65 \\
(18.38 \\
39.34)\end{array}$ & 8.951273 & 34.50784 \\
\hline & & 06 Trarza & $\begin{array}{l}52.77 \\
(43.43 \\
61.93)\end{array}$ & 10.18605 & 34.50784 \\
\hline & & 07 Adrar & $\begin{array}{l}34.42 \\
(24.45 \\
45.97)\end{array}$ & 0.381213 & 34.50784 \\
\hline
\end{tabular}




\begin{tabular}{|c|c|c|c|c|c|}
\hline Country & Year & Subgroup & $\begin{array}{l}\text { Estimate } \\
(95 \% \mathrm{Cl})\end{array}$ & Population & $\begin{array}{l}\text { National } \\
\text { Coverage }\end{array}$ \\
\hline & & 08 Dakhlett Nouadibou & $\begin{array}{l}47.97 \\
(27.99 \\
68.62)\end{array}$ & 0.766553 & 34.50784 \\
\hline & & 09 Tagant & $\begin{array}{l}33.17 \\
(26.89 \\
40.10)\end{array}$ & 0.701618 & 34.50784 \\
\hline & & 10 Guidimagha & $\begin{array}{l}34.68 \\
(28.82 \\
41.05)\end{array}$ & 15.04826 & 34.50784 \\
\hline & & 11 Tirs-ezemour & $\begin{array}{l}41.63 \\
(30.20 \\
54.04)\end{array}$ & 0.41333 & 34.50784 \\
\hline & & 12 Inchiri & $\begin{array}{l}29.13 \\
(18.05 \\
43.42)\end{array}$ & 0.189108 & 34.50784 \\
\hline & & 13 Nouakchott & $\begin{array}{l}36.60 \\
(28.01 \\
46.13)\end{array}$ & 13.92776 & 34.50784 \\
\hline \multirow[t]{7}{*}{ Mozambique } & 2015 & 01 niassa & $\begin{array}{l}41.33 \\
(31.04 \\
52.44)\end{array}$ & 10.49117 & 43.02846 \\
\hline & & 02 cabo delgado & $\begin{array}{l}56.41 \\
(35.05 \\
75.63)\end{array}$ & 8.079485 & 43.02846 \\
\hline & & 03 nampula & $\begin{array}{l}32.29 \\
(20.31 \\
47.15)\end{array}$ & 22.62527 & 43.02846 \\
\hline & & 04 zambezia & $\begin{array}{l}30.81 \\
(18.70 \\
46.29)\end{array}$ & 21.03875 & 43.02846 \\
\hline & & 05 tete & $\begin{array}{l}67.58 \\
(49.94 \\
81.32)\end{array}$ & 5.256539 & 43.02846 \\
\hline & & 06 manica & $\begin{array}{l}63.54 \\
(42.86 \\
80.19)\end{array}$ & 6.577499 & 43.02846 \\
\hline & & 07 sofala & $\begin{array}{l}44.23 \\
(26.86 \\
63.13)\end{array}$ & 7.571609 & 43.02846 \\
\hline
\end{tabular}




\begin{tabular}{|c|c|c|c|c|c|}
\hline Country & Year & Subgroup & $\begin{array}{l}\text { Estimate } \\
(95 \% \mathrm{Cl})\end{array}$ & Population & $\begin{array}{l}\text { National } \\
\text { Coverage }\end{array}$ \\
\hline & & 08 inhambane & $\begin{array}{l}47.37 \\
(27.41 \\
68.20)\end{array}$ & 3.621083 & 43.02846 \\
\hline & & 09 gaza & $\begin{array}{l}52.33 \\
(39.32, \\
65.03)\end{array}$ & 8.408001 & 43.02846 \\
\hline & & 10 maputo provincia & NA & 3.171028 & 43.02846 \\
\hline & & 11 maputo cidade & $\begin{array}{l}56.91 \\
(33.81 \\
77.35)\end{array}$ & 3.159564 & 43.02846 \\
\hline \multirow[t]{10}{*}{ Namibia } & 2013 & 01 caprivi & $\begin{array}{l}54.57 \\
(45.25 \\
63.58)\end{array}$ & 11.26575 & 51.98061 \\
\hline & & 02 erongo & $\begin{array}{l}42.90 \\
(28.59 \\
58.51)\end{array}$ & 4.048872 & 51.98061 \\
\hline & & 03 hardap & NA & 1.547992 & 51.98061 \\
\hline & & 04 karas & $\begin{array}{l}50.76 \\
(34.96 \\
66.41)\end{array}$ & 1.910572 & 51.98061 \\
\hline & & 05 kavango & $\begin{array}{l}61.16 \\
(52.07 \\
69.53)\end{array}$ & 21.50312 & 51.98061 \\
\hline & & 06 khomas & $\begin{array}{l}49.49 \\
(39.39 \\
59.64)\end{array}$ & 17.55895 & 51.98061 \\
\hline & & 07 kunene & $\begin{array}{l}45.92 \\
(33.37 \\
59.01)\end{array}$ & 2.633852 & 51.98061 \\
\hline & & 08 ohangwena & $\begin{array}{l}38.81 \\
(26.39 \\
52.88)\end{array}$ & 10.49002 & 51.98061 \\
\hline & & 09 omaheke & $\begin{array}{l}55.07 \\
(38.61 \\
70.48)\end{array}$ & 2.61765 & 51.98061 \\
\hline & & 10 omusati & $\begin{array}{l}50.93 \\
(38.51 \\
63.24)\end{array}$ & 10.56234 & 51.98061 \\
\hline
\end{tabular}




\begin{tabular}{|c|c|c|c|c|c|}
\hline Country & Year & Subgroup & $\begin{array}{l}\text { Estimate } \\
(95 \% \mathrm{Cl})\end{array}$ & Population & $\begin{array}{l}\text { National } \\
\text { Coverage }\end{array}$ \\
\hline & & 11 oshana & $\begin{array}{l}44.11 \\
(23.75, \\
66.66)\end{array}$ & 3.830878 & 51.98061 \\
\hline & & 12 oshikoto & $\begin{array}{l}54.48 \\
(43.30 \\
65.22)\end{array}$ & 6.493157 & 51.98061 \\
\hline & & 13 otjozondjupa & $\begin{array}{l}53.89 \\
(38.38, \\
68.68)\end{array}$ & 5.536846 & 51.98061 \\
\hline \multirow[t]{8}{*}{ Niger } & 2012 & 01 Agadez & $\begin{array}{l}42.97 \\
(30.16 \\
56.80)\end{array}$ & 1.582355 & 42.96736 \\
\hline & & 02 Diffa & $\begin{array}{l}55.94 \\
(43.64, \\
67.54)\end{array}$ & 1.711278 & 42.96736 \\
\hline & & 03 Dosso & $\begin{array}{l}49.55 \\
(40.60 \\
58.53)\end{array}$ & 10.28877 & 42.96736 \\
\hline & & 04 Maradi & $\begin{array}{l}43.12 \\
(35.35 \\
51.24)\end{array}$ & 17.09726 & 42.96736 \\
\hline & & 05 Tahoua & $\begin{array}{l}33.96 \\
(26.55 \\
42.26)\end{array}$ & 20.99217 & 42.96736 \\
\hline & & 06 Tillaberi & $\begin{array}{l}46.87 \\
(38.55 \\
55.37)\end{array}$ & 13.38614 & 42.96736 \\
\hline & & 07 Zinder & $\begin{array}{l}43.28 \\
(35.88, \\
51.00)\end{array}$ & 25.4627 & 42.96736 \\
\hline & & 08 Niamey & $\begin{array}{l}46.75 \\
(37.22 \\
56.52)\end{array}$ & 9.479325 & 42.96736 \\
\hline \multirow[t]{2}{*}{ Nigeria } & 2013 & 01 North Central & $\begin{array}{l}37.40 \\
(28.69, \\
47.00)\end{array}$ & 9.943455 & 29.23584 \\
\hline & & 02 North East & $\begin{array}{l}23.13 \\
(19.44 \\
27.29)\end{array}$ & 35.77472 & 29.23584 \\
\hline
\end{tabular}




\begin{tabular}{|c|c|c|c|c|c|}
\hline Country & Year & Subgroup & $\begin{array}{l}\text { Estimate } \\
(95 \% \mathrm{Cl})\end{array}$ & Population & $\begin{array}{l}\text { National } \\
\text { Coverage }\end{array}$ \\
\hline & & 03 North West & $\begin{array}{l}26.84 \\
(22.90 \\
31.18)\end{array}$ & 32.39882 & 29.23584 \\
\hline & & 04 South East & $\begin{array}{l}34.99 \\
(27.30 \\
43.56)\end{array}$ & 8.963924 & 29.23584 \\
\hline & & 05 South South & $\begin{array}{l}36.72 \\
(29.04 \\
45.13)\end{array}$ & 4.1927 & 29.23584 \\
\hline & & 06 South West & $\begin{array}{l}44.30 \\
(37.12, \\
51.72)\end{array}$ & 8.72638 & 29.23584 \\
\hline \multirow[t]{5}{*}{ Rwanda } & 2014 & 01 kigali city & $\begin{array}{l}17.46 \\
(10.86 \\
26.87)\end{array}$ & 8.018152 & 19.46708 \\
\hline & & 02 south & $\begin{array}{l}17.14 \\
(12.26, \\
23.44)\end{array}$ & 23.16374 & 19.46708 \\
\hline & & 03 west & $\begin{array}{l}18.27 \\
(13.26, \\
24.62)\end{array}$ & 29.28994 & 19.46708 \\
\hline & & 04 north & $\begin{array}{l}21.80 \\
(14.17 \\
32.01)\end{array}$ & 12.61922 & 19.46708 \\
\hline & & 05 east & $\begin{array}{l}22.26 \\
(16.43, \\
29.43)\end{array}$ & 26.90895 & 19.46708 \\
\hline \multirow[t]{4}{*}{$\begin{array}{l}\text { Sao Tome and } \\
\text { Principe }\end{array}$} & 2014 & 01 Regiaao Centro Este & $\begin{array}{l}60.01 \\
(50.95 \\
68.44)\end{array}$ & 62.0037 & 61.02909 \\
\hline & & 02 Regiaao Norte Oeste & $\begin{array}{l}62.23 \\
(48.73 \\
74.07)\end{array}$ & 19.53131 & 61.02909 \\
\hline & & 03 Regiaao Sul Este & $\begin{array}{l}63.96 \\
\text { (54.51, } \\
72.44)\end{array}$ & 14.41731 & 61.02909 \\
\hline & & $\begin{array}{l}04 \text { Regiaao Autonoma de } \\
\text { Principe }\end{array}$ & $\begin{array}{l}60.23 \\
(43.43 \\
74.92)\end{array}$ & 4.047688 & 61.02909 \\
\hline
\end{tabular}




\begin{tabular}{|c|c|c|c|c|c|}
\hline Country & Year & Subgroup & $\begin{array}{l}\text { Estimate } \\
(95 \% \mathrm{Cl})\end{array}$ & Population & $\begin{array}{l}\text { National } \\
\text { Coverage }\end{array}$ \\
\hline \multirow[t]{13}{*}{ Senegal } & \multirow[t]{13}{*}{2014} & 01 dakar & $\begin{array}{l}52.18 \\
(35.45 \\
68.44)\end{array}$ & 14.58356 & 48.59681 \\
\hline & & 02 ziguinchor & $\begin{array}{l}42.41 \\
(23.86, \\
63.38)\end{array}$ & 3.355799 & 48.59681 \\
\hline & & 03 diourbel & $\begin{array}{l}64.72 \\
(57.08, \\
71.67)\end{array}$ & 10.51733 & 48.59681 \\
\hline & & 04 saint-louis & $\begin{array}{l}35.19 \\
(29.51 \\
41.33)\end{array}$ & 13.48023 & 48.59681 \\
\hline & & 05 tambacounda & $\begin{array}{l}62.79 \\
(41.90 \\
79.79)\end{array}$ & 4.593456 & 48.59681 \\
\hline & & 06 kaolack & $\begin{array}{l}49.93 \\
(34.76 \\
65.11)\end{array}$ & 8.280867 & 48.59681 \\
\hline & & 07 thies & $\begin{array}{l}46.47 \\
(36.69, \\
56.53)\end{array}$ & 12.85262 & 48.59681 \\
\hline & & 08 louga & $\begin{array}{l}37.11 \\
(25.51 \\
50.42)\end{array}$ & 6.974087 & 48.59681 \\
\hline & & 09 fatick & $\begin{array}{l}46.95 \\
(35.99 \\
58.21)\end{array}$ & 5.475001 & 48.59681 \\
\hline & & 10 kolda & $\begin{array}{l}54.50 \\
(38.69 \\
69.45)\end{array}$ & 4.882447 & 48.59681 \\
\hline & & 11 matam & $\begin{array}{l}38.54 \\
(28.67 \\
49.46)\end{array}$ & 5.251358 & 48.59681 \\
\hline & & 12 kaffrine & $\begin{array}{l}51.00 \\
(33.95, \\
67.82)\end{array}$ & 4.587029 & 48.59681 \\
\hline & & 13 kedougou & $\begin{array}{l}47.42 \\
(34.19 \\
61.02)\end{array}$ & 1.359931 & 48.59681 \\
\hline
\end{tabular}




\begin{tabular}{|c|c|c|c|c|c|}
\hline Country & Year & Subgroup & $\begin{array}{l}\text { Estimate } \\
(95 \% \mathrm{Cl})\end{array}$ & Population & $\begin{array}{l}\text { National } \\
\text { Coverage }\end{array}$ \\
\hline & & 14 sedhiou & $\begin{array}{l}57.52 \\
(46.38 \\
67.93)\end{array}$ & 3.806289 & 48.59681 \\
\hline \multirow[t]{4}{*}{ Sierra Leone } & \multirow[t]{4}{*}{2013} & 01 Eastern & $\begin{array}{l}55.74 \\
(47.71, \\
63.49)\end{array}$ & 19.94911 & 57.57013 \\
\hline & & 02 Northern & $\begin{array}{l}56.80 \\
(51.80 \\
61.67)\end{array}$ & 48.02768 & 57.57013 \\
\hline & & 03 Southern & $\begin{array}{l}58.64 \\
(49.00 \\
67.65)\end{array}$ & 17.61435 & 57.57013 \\
\hline & & 04 Western & $\begin{array}{l}61.33 \\
(52.51 \\
69.46)\end{array}$ & 14.40887 & 57.57013 \\
\hline \multirow[t]{6}{*}{ Togo } & \multirow[t]{6}{*}{2013} & $\begin{array}{l}01 \text { grande agglomeration } \\
\text { de lome }\end{array}$ & $\begin{array}{l}31.04 \\
(21.72, \\
42.20)\end{array}$ & 15.55007 & 33.42139 \\
\hline & & $\begin{array}{l}02 \text { maritime (sans } \\
\text { agglomeration de lome) }\end{array}$ & $\begin{array}{l}13.43(7.57 \\
22.70)\end{array}$ & 14.08151 & 33.42139 \\
\hline & & 03 plateaux & $\begin{array}{l}35.04 \\
(29.39 \\
41.14)\end{array}$ & 27.50038 & 33.42139 \\
\hline & & 04 centrale & $\begin{array}{l}34.56 \\
(27.22, \\
42.71)\end{array}$ & 9.950897 & 33.42139 \\
\hline & & 05 kara & $\begin{array}{l}41.28 \\
(33.63 \\
49.38)\end{array}$ & 14.19173 & 33.42139 \\
\hline & & 06 savanes & $\begin{array}{l}41.47 \\
(33.97 \\
49.39)\end{array}$ & 18.72541 & 33.42139 \\
\hline \multirow[t]{3}{*}{ Uganda } & \multirow[t]{3}{*}{2016} & 01 kampala & $\begin{array}{l}35.85 \\
(26.00 \\
47.05)\end{array}$ & 3.028061 & 37.38395 \\
\hline & & 02 central 1 & $\begin{array}{l}47.24 \\
(37.91 \\
56.77)\end{array}$ & 12.68701 & 37.38395 \\
\hline & & 03 central2 & $\begin{array}{l}38.78 \\
(30.34 \\
47.96)\end{array}$ & 9.053208 & 37.38395 \\
\hline
\end{tabular}




\begin{tabular}{|c|c|c|c|c|c|}
\hline Country & Year & Subgroup & $\begin{array}{l}\text { Estimate } \\
(95 \% \mathrm{Cl})\end{array}$ & Population & $\begin{array}{l}\text { National } \\
\text { Coverage }\end{array}$ \\
\hline & & 04 busoga & $\begin{array}{l}33.10 \\
(29.40 \\
37.02)\end{array}$ & 13.78741 & 37.38395 \\
\hline & & 05 bukedi & $\begin{array}{l}45.41 \\
(39.12, \\
51.85)\end{array}$ & 6.423967 & 37.38395 \\
\hline & & 06 bugishu & $\begin{array}{l}29.62 \\
(21.73, \\
38.95)\end{array}$ & 3.694834 & 37.38395 \\
\hline & & 07 teso & $\begin{array}{l}21.03 \\
(17.71, \\
24.79)\end{array}$ & 9.388466 & 37.38395 \\
\hline & & 08 karamoja & $\begin{array}{l}55.66 \\
(43.96 \\
66.75)\end{array}$ & 3.335683 & 37.38395 \\
\hline & & 09 lango & $\begin{array}{l}21.96 \\
(16.02, \\
29.35)\end{array}$ & 5.540022 & 37.38395 \\
\hline & & 10 acholi & $\begin{array}{l}34.58 \\
(27.79, \\
42.06)\end{array}$ & 6.139533 & 37.38395 \\
\hline & & 11 west nile & $\begin{array}{l}41.77 \\
(33.39 \\
50.64)\end{array}$ & 5.612174 & 37.38395 \\
\hline & & 12 bunyoro & $\begin{array}{l}49.75 \\
(41.12, \\
58.40)\end{array}$ & 3.009143 & 37.38395 \\
\hline & & 13 tooro & $\begin{array}{l}45.98 \\
(37.96 \\
54.22)\end{array}$ & 8.835254 & 37.38395 \\
\hline & & 14 ankole & $\begin{array}{l}26.05 \\
(19.36 \\
34.08)\end{array}$ & 6.763901 & 37.38395 \\
\hline & & 15 kigezi & $\begin{array}{l}50.98 \\
(41.42 \\
60.46)\end{array}$ & 2.701337 & 37.38395 \\
\hline \multirow{3}{*}{$\begin{array}{l}\text { United Republic of } \\
\text { Tanzania }\end{array}$} & \multirow[t]{3}{*}{2015} & 01 dodoma & NA & 3.441052 & 45.45398 \\
\hline & & 02 arusha & NA & 2.801291 & 45.45398 \\
\hline & & 03 kilimanjaro & NA & 1.476599 & 45.45398 \\
\hline
\end{tabular}




\begin{tabular}{|c|c|c|c|c|c|}
\hline Country & Year & Subgroup & $\begin{array}{l}\text { Estimate } \\
(95 \% \mathrm{Cl})\end{array}$ & Population & $\begin{array}{l}\text { National } \\
\text { Coverage }\end{array}$ \\
\hline & & 04 tanga & NA & 2.170607 & 45.45398 \\
\hline & & 05 morogoro & NA & 3.174978 & 45.45398 \\
\hline & & 06 pwani & NA & 1.788528 & 45.45398 \\
\hline & & 07 dar es salaam & $\begin{array}{l}55.82 \\
(38.61 \\
71.73)\end{array}$ & 9.608987 & 45.45398 \\
\hline & & 08 lindi & $\begin{array}{l}44.13 \\
(28.60 \\
60.89)\end{array}$ & 2.090084 & 45.45398 \\
\hline & & 09 mtwara & $\begin{array}{l}60.85 \\
(47.09 \\
73.07)\end{array}$ & 3.327305 & 45.45398 \\
\hline & & 10 ruvuma & $\begin{array}{l}45.86 \\
(26.91 \\
66.09)\end{array}$ & 2.459604 & 45.45398 \\
\hline & & 11 iringa & NA & 1.10595 & 45.45398 \\
\hline & & 12 mbeya & $\begin{array}{l}54.93 \\
(35.27 \\
73.15)\end{array}$ & 6.471929 & 45.45398 \\
\hline & & 13 singida & $\begin{array}{l}54.45 \\
(33.94 \\
73.56)\end{array}$ & 2.749101 & 45.45398 \\
\hline & & 14 tabora & $\begin{array}{l}40.53 \\
(16.58 \\
70.02)\end{array}$ & 3.154757 & 45.45398 \\
\hline & & 15 rukwa & $\begin{array}{l}20.95 \\
(13.07 \\
31.84)\end{array}$ & 4.538253 & 45.45398 \\
\hline & & 16 kigoma & $\begin{array}{l}37.83 \\
(21.31 \\
57.76)\end{array}$ & 8.96169 & 45.45398 \\
\hline & & 17 shinyanga & $\begin{array}{l}63.10 \\
(35.92 \\
83.92)\end{array}$ & 4.439744 & 45.45398 \\
\hline & & 18 kagera & $\begin{array}{l}47.73 \\
(31.12 \\
64.85)\end{array}$ & 4.849433 & 45.45398 \\
\hline
\end{tabular}




\begin{tabular}{|c|c|c|c|c|c|}
\hline Country & Year & Subgroup & $\begin{array}{l}\text { Estimate } \\
(95 \% \mathrm{Cl})\end{array}$ & Population & $\begin{array}{l}\text { National } \\
\text { Coverage }\end{array}$ \\
\hline & & 19 mwanza & $\begin{array}{l}45.37 \\
(29.06 \\
62.74)\end{array}$ & 7.271308 & 45.45398 \\
\hline & & 20 mara & $\begin{array}{l}44.07 \\
(37.07 \\
51.31)\end{array}$ & 6.698983 & 45.45398 \\
\hline & & 21 manyara & $\begin{array}{l}37.64 \\
(26.03 \\
50.87)\end{array}$ & 3.469363 & 45.45398 \\
\hline & & 22 njombe & NA & 1.091423 & 45.45398 \\
\hline & & 23 katavi & $\begin{array}{l}29.77 \\
(16.93 \\
46.84)\end{array}$ & 1.58284 & 45.45398 \\
\hline & & 24 simiyu & $\begin{array}{l}41.27 \\
(31.63 \\
51.62)\end{array}$ & 5.497825 & 45.45398 \\
\hline & & 25 geita & $\begin{array}{l}50.62 \\
(37.14 \\
64.02)\end{array}$ & 3.424703 & 45.45398 \\
\hline & & 26 kaskazini unguja & $\begin{array}{l}36.74 \\
(22.75 \\
53.38)\end{array}$ & 0.515052 & 45.45398 \\
\hline & & 27 kusini unguja & NA & 0.169818 & 45.45398 \\
\hline & & 28 mjini magharibi & $\begin{array}{l}54.74 \\
(31.71 \\
75.91)\end{array}$ & 0.750424 & 45.45398 \\
\hline & & 29 kaskazini pemba & $\begin{array}{l}39.03 \\
(22.58 \\
58.42)\end{array}$ & 0.546524 & 45.45398 \\
\hline & & 30 kusini pemba & $\begin{array}{l}38.95 \\
(20.16 \\
61.72)\end{array}$ & 0.371845 & 45.45398 \\
\hline \multirow[t]{2}{*}{ Zambia } & 2013 & 01 central & $\begin{array}{l}44.21 \\
(35.39 \\
53.41)\end{array}$ & 8.190496 & 56.17617 \\
\hline & & 02 copperbelt & $\begin{array}{l}57.13 \\
(47.93 \\
65.86)\end{array}$ & 16.14709 & 56.17617 \\
\hline
\end{tabular}




\begin{tabular}{|c|c|c|c|c|c|}
\hline Country & Year & Subgroup & $\begin{array}{l}\text { Estimate } \\
(95 \% \mathrm{Cl})\end{array}$ & Population & $\begin{array}{l}\text { National } \\
\text { Coverage }\end{array}$ \\
\hline & & 03 eastern & $\begin{array}{l}57.16 \\
(48.28 \\
65.60)\end{array}$ & 11.88424 & 56.17617 \\
\hline & & 04 luapula & $\begin{array}{l}58.97 \\
(51.43 \\
66.12)\end{array}$ & 8.030398 & 56.17617 \\
\hline & & 05 lusaka & $\begin{array}{l}64.21 \\
(55.30 \\
72.23)\end{array}$ & 14.14885 & 56.17617 \\
\hline & & 06 muchinga & $\begin{array}{l}57.22 \\
(48.95 \\
65.11)\end{array}$ & 6.334013 & 56.17617 \\
\hline & & 07 northern & $\begin{array}{l}46.76 \\
(37.42 \\
56.33)\end{array}$ & 9.41377 & 56.17617 \\
\hline & & 08 north western & $\begin{array}{l}58.10 \\
(49.01 \\
66.68)\end{array}$ & 4.767902 & 56.17617 \\
\hline & & 09 southern & $\begin{array}{l}57.28 \\
(50.05 \\
64.20)\end{array}$ & 15.51533 & 56.17617 \\
\hline & & 10 western & $\begin{array}{l}54.41 \\
(44.62 \\
63.87)\end{array}$ & 5.567904 & 56.17617 \\
\hline \multirow[t]{5}{*}{ Zimbabwe } & \multirow[t]{5}{*}{2014} & 01 Bulawayo & $\begin{array}{l}59.57 \\
(45.01 \\
72.62)\end{array}$ & 4.061207 & 56.36424 \\
\hline & & 02 Manicaland & $\begin{array}{l}66.48 \\
(58.91 \\
73.29)\end{array}$ & 14.25693 & 56.36424 \\
\hline & & 03 Mashonaland Central & $\begin{array}{l}57.93 \\
(50.11 \\
65.36)\end{array}$ & 6.62444 & 56.36424 \\
\hline & & 04 Mashonaland East & $\begin{array}{l}58.70 \\
(50.86 \\
66.13)\end{array}$ & 10.46592 & 56.36424 \\
\hline & & 05 Mashonaland West & $\begin{array}{l}53.68 \\
(47.71 \\
59.55)\end{array}$ & 14.59176 & 56.36424 \\
\hline
\end{tabular}




\begin{tabular}{|c|c|c|c|c|c|}
\hline Country & Year & Subgroup & $\begin{array}{l}\text { Estimate } \\
(95 \% \mathrm{Cl})\end{array}$ & Population & $\begin{array}{l}\text { National } \\
\text { Coverage }\end{array}$ \\
\hline & & 06 Matabeleland North & $\begin{array}{l}56.43 \\
(46.40 \\
65.96)\end{array}$ & 6.341551 & 56.36424 \\
\hline & & 07 Matabeleland South & $\begin{array}{l}72.91 \\
(61.49, \\
81.94)\end{array}$ & 6.127159 & 56.36424 \\
\hline & & 08 Midlands & $\begin{array}{l}49.66 \\
(41.98, \\
57.36)\end{array}$ & 13.00898 & 56.36424 \\
\hline & & 09 Masvingo & $\begin{array}{l}43.84 \\
(37.00 \\
50.92)\end{array}$ & 15.12306 & 56.36424 \\
\hline & & 10 Harare & $\begin{array}{l}58.65 \\
(50.48, \\
66.36)\end{array}$ & 9.398988 & 56.36424 \\
\hline
\end{tabular}


Table 10

Sub-national regional inequality in ORT and continued feeding in the 35 low income, lower middle income and upper middle income SSA countries

\begin{tabular}{|c|c|c|c|c|c|}
\hline \multirow[t]{2}{*}{ Country } & \multirow[t]{2}{*}{ Year } & \multicolumn{4}{|c|}{ Type and value of inequality measure with $95 \% \mathrm{Cl}$} \\
\hline & & D & $\mathbf{R}$ & PAR & PAF \\
\hline Ghana & 2014 & $\begin{array}{l}53.08(25.42 \\
80.74)\end{array}$ & $\begin{array}{l}3.44(0.26 \\
6.63)\end{array}$ & $\begin{array}{l}33.25(17.22 \\
49.28)\end{array}$ & $\begin{array}{l}80.12(41.48 \\
118.75)\end{array}$ \\
\hline Algeria & 2012 & $\begin{array}{l}18.88(2.86 \\
34.90)\end{array}$ & $\begin{array}{l}1.58(0.91, \\
2.24)\end{array}$ & $\begin{array}{l}9.69(-3.76, \\
23.16)\end{array}$ & $\begin{array}{l}23.24(-9.02 \\
55.50)\end{array}$ \\
\hline Angola & 2015 & $\begin{array}{l}51.34(42.47 \\
60.21)\end{array}$ & $\begin{array}{l}\text { 14.94(-2.88, } \\
32.77)\end{array}$ & $\begin{array}{l}15.33(1.30 \\
29.37)\end{array}$ & $\begin{array}{l}38.64(3.28 \\
74.00)\end{array}$ \\
\hline Benin & 2014 & $\begin{array}{l}27.24(11.67 \\
42.81)\end{array}$ & $\begin{array}{l}2.42(0.92 \\
3.91)\end{array}$ & $\begin{array}{l}13.86(5.78 \\
21.94)\end{array}$ & $\begin{array}{l}42.58(17.76 \\
67.40)\end{array}$ \\
\hline Burundi & 2016 & $\begin{array}{l}32.31(17.08 \\
47.54)\end{array}$ & $\begin{array}{l}3.05(1.22 \\
4.87)\end{array}$ & $\begin{array}{l}13.09(4.52 \\
21.66)\end{array}$ & $\begin{array}{l}37.47(12.95 \\
61.99)\end{array}$ \\
\hline Cameroon & 2014 & $\begin{array}{l}29.81(9.46 \\
50.16)\end{array}$ & $\begin{array}{l}1.90(1.20 \\
2.60)\end{array}$ & $\begin{array}{l}23.51(20.56 \\
26.45)\end{array}$ & $\begin{array}{l}59.84(52.34 \\
67.33)\end{array}$ \\
\hline Chad & 2014 & $\begin{array}{l}25.50(11.09 \\
39.92)\end{array}$ & $\begin{array}{l}3.15(0.71 \\
5.58)\end{array}$ & $\begin{array}{l}8.48(2.42, \\
14.54)\end{array}$ & $\begin{array}{l}29.36(8.38 \\
50.35)\end{array}$ \\
\hline Comoros & 2012 & $\begin{array}{l}8.48(-8.63 \\
25.61)\end{array}$ & $\begin{array}{l}1.17(0.78 \\
1.56)\end{array}$ & $\begin{array}{l}3.18(-14.49 \\
20.87)\end{array}$ & $\begin{array}{l}5.90(-26.87 \\
38.69)\end{array}$ \\
\hline Congo & 2014 & $\begin{array}{l}24.43(11.58 \\
37.27)\end{array}$ & $\begin{array}{l}2.00(1.17 \\
2.82)\end{array}$ & $\begin{array}{l}6.57(-4.09 \\
17.24)\end{array}$ & $\begin{array}{l}15.56(-9.70 \\
40.82)\end{array}$ \\
\hline $\begin{array}{l}\text { Democratic Republic of } \\
\text { the Congo }\end{array}$ & 2013 & $\begin{array}{l}25.99(11.03 \\
40.94)\end{array}$ & $\begin{array}{l}1.79(1.17 \\
2.42)\end{array}$ & $\begin{array}{l}18.61(12.36 \\
24.85)\end{array}$ & $\begin{array}{l}46.58(30.95 \\
62.22)\end{array}$ \\
\hline Eswatini & 2014 & $\begin{array}{l}28.80(13.38 \\
44.23)\end{array}$ & $\begin{array}{l}1.86(1.18 \\
2.55)\end{array}$ & $\begin{array}{l}15.62(6.93 \\
24.32)\end{array}$ & $\begin{array}{l}33.70(14.94 \\
52.46)\end{array}$ \\
\hline Ethiopia & 2016 & $\begin{array}{l}38.28(22.44, \\
54.12)\end{array}$ & $\begin{array}{l}2.86(1.63 \\
4.09)\end{array}$ & $\begin{array}{l}30.16(2.41 \\
57.91)\end{array}$ & $\begin{array}{l}105.26(8.43 \\
202.09)\end{array}$ \\
\hline Gabon & 2012 & $\begin{array}{l}25.87(-1.11 \\
52.86)\end{array}$ & $\begin{array}{l}1.68(0.54 \\
2.82)\end{array}$ & $\begin{array}{l}11.67(-28.90 \\
52.26)\end{array}$ & $\begin{array}{l}22.49(-55.69, \\
100.68)\end{array}$ \\
\hline Gambia & 2013 & $\begin{array}{l}17.04(0.50 \\
33.58)\end{array}$ & $\begin{array}{l}1.42(0.93 \\
1.91)\end{array}$ & $\begin{array}{l}6.42(-3.30 \\
16.15)\end{array}$ & $\begin{array}{l}12.63(-6.50 \\
31.77)\end{array}$ \\
\hline Guinea & 2016 & $\begin{array}{l}45.96(29.20 \\
62.71)\end{array}$ & $\begin{array}{l}4.43(1.94 \\
6.93)\end{array}$ & $\begin{array}{l}15.14(8.94 \\
21.34)\end{array}$ & $\begin{array}{l}34.28(20.24 \\
48.32)\end{array}$ \\
\hline Guinea-Bissau & 2014 & $\begin{array}{l}59.62(45.91 \\
73.32)\end{array}$ & $\begin{array}{l}4.84(2.21, \\
7.47)\end{array}$ & $\begin{array}{l}20.51(13.88 \\
27.15)\end{array}$ & $\begin{array}{l}37.57(25.42 \\
49.71)\end{array}$ \\
\hline
\end{tabular}




\begin{tabular}{|c|c|c|c|c|c|}
\hline \multirow[t]{2}{*}{ Country } & \multirow[t]{2}{*}{ Year } & \multicolumn{4}{|c|}{ Type and value of inequality measure with $95 \% \mathrm{Cl}$} \\
\hline & & D & $\mathbf{R}$ & PAR & PAF \\
\hline Kenya & 2014 & $\begin{array}{l}40.92(27.23 \\
54.61)\end{array}$ & $\begin{array}{l}2.30(1.49 \\
3.10)\end{array}$ & $\begin{array}{l}27.22(13.88 \\
40.56)\end{array}$ & $\begin{array}{l}60.31(30.76 \\
89.86)\end{array}$ \\
\hline Lesotho & 2014 & NA & NA & NA & PAR \\
\hline Liberia & 2013 & $\begin{array}{l}14.01(5.12 \\
22.90)\end{array}$ & $\begin{array}{l}1.35(1.10, \\
1.61)\end{array}$ & $\begin{array}{l}6.52(-1.61, \\
14.67)\end{array}$ & $\begin{array}{l}14.06(-3.48 \\
31.60)\end{array}$ \\
\hline Malawi & 2015 & $\begin{array}{l}14.78(6.36 \\
23.20)\end{array}$ & $\begin{array}{l}1.34(1.08, \\
1.59)\end{array}$ & $\begin{array}{l}1.48(-3.55 \\
6.53)\end{array}$ & $\begin{array}{l}2.62(-6.27 \\
11.53)\end{array}$ \\
\hline Mali & 2015 & $\begin{array}{l}28.85(20.09 \\
37.60)\end{array}$ & $\begin{array}{l}2.50(1.74 \\
3.26)\end{array}$ & $\begin{array}{l}19.30(16.53 \\
22.07)\end{array}$ & $\begin{array}{l}67.25(57.60 \\
76.90)\end{array}$ \\
\hline Mauritania & 2015 & $\begin{array}{l}29.27(16.31 \\
42.23)\end{array}$ & $\begin{array}{l}2.24(1.29 \\
3.19)\end{array}$ & $\begin{array}{l}\text { 18.27 (11.78, } \\
24.70)\end{array}$ & $\begin{array}{l}52.94(34.15, \\
71.73)\end{array}$ \\
\hline Mozambique & 2015 & NA & NA & NA & NA \\
\hline Namibia & 2013 & NA & NA & NA & NA \\
\hline Niger & 2012 & $\begin{array}{l}21.9797 .48 \\
36.45)\end{array}$ & $\begin{array}{l}1.64(1.12 \\
2.17)\end{array}$ & $\begin{array}{l}12.97(8.57 \\
17.36)\end{array}$ & $\begin{array}{l}30.19(19.96 \\
40.42)\end{array}$ \\
\hline Nigeria & 2013 & $\begin{array}{l}21.16(12.84 \\
29.48)\end{array}$ & $\begin{array}{l}1.91(1.46, \\
2.36)\end{array}$ & $\begin{array}{l}15.06(12.94 \\
17.18)\end{array}$ & $\begin{array}{l}51.52(44.28 \\
58.77)\end{array}$ \\
\hline Rwanda & 2014 & $\begin{array}{l}5.12(-3.40 \\
13.65)\end{array}$ & $\begin{array}{l}1.29(0.73 \\
1.86)\end{array}$ & $\begin{array}{l}2.80(-1.72 \\
7.32)\end{array}$ & $\begin{array}{l}14.38(-8.84 \\
37.61)\end{array}$ \\
\hline Sao Tome and Principe & 2014 & $\begin{array}{l}3.95(-8.54 \\
16.44)\end{array}$ & $\begin{array}{l}1.06(0.85 \\
1.28)\end{array}$ & $\begin{array}{l}2.93(-1.00 \\
6.88)\end{array}$ & $\begin{array}{l}4.81(-1.64 \\
11.27)\end{array}$ \\
\hline Senegal & 2014 & $\begin{array}{l}29.52(20.15 \\
38.89)\end{array}$ & $\begin{array}{l}1.83(1.46, \\
2.20)\end{array}$ & $\begin{array}{l}16.12(8.96 \\
23.20)\end{array}$ & $\begin{array}{l}33.18 \text { (18.44, } \\
47.92)\end{array}$ \\
\hline Sierra Leone & 2013 & $\begin{array}{l}5.58(-6.04 \\
17.22)\end{array}$ & $\begin{array}{l}1.10(0.88, \\
1.31)\end{array}$ & $\begin{array}{l}3.76(-1.86, \\
9.39)\end{array}$ & $\begin{array}{l}6.54(-3.23 \\
16.32)\end{array}$ \\
\hline Togo & 2013 & $\begin{array}{l}28.04(17.35 \\
38.73)\end{array}$ & $\begin{array}{l}3.08(1.29 \\
4.88)\end{array}$ & $\begin{array}{l}8.05(2.30 \\
13.80)\end{array}$ & $\begin{array}{l}24.09 \text { (6.88, } \\
41.30)\end{array}$ \\
\hline Uganda & 2016 & $\begin{array}{l}34.62(22.52 \\
46.72)\end{array}$ & $\begin{array}{l}2.64(1.93, \\
3.35)\end{array}$ & $\begin{array}{l}18.27(13.49 \\
23.05)\end{array}$ & $\begin{array}{l}48.89(36.10, \\
61.67)\end{array}$ \\
\hline $\begin{array}{l}\text { United Republic of } \\
\text { Tanzania }\end{array}$ & 2015 & NA & NA & NA & NA \\
\hline Zambia & 2013 & $\begin{array}{l}19.99(7.53 \\
32.45)\end{array}$ & $\begin{array}{l}1.45(1.09, \\
1.80)\end{array}$ & $\begin{array}{l}8.03(0.75 \\
15.31)\end{array}$ & $\begin{array}{l}14.30(1.34 \\
27.26)\end{array}$ \\
\hline Zimbabwe & 2014 & $\begin{array}{l}29.07(16.62, \\
41.51)\end{array}$ & $\begin{array}{l}1.66(1.30, \\
2.01)\end{array}$ & $\begin{array}{l}16.55(10.61 \\
22.48)\end{array}$ & $\begin{array}{l}29.36(18.83 \\
39.89)\end{array}$ \\
\hline
\end{tabular}




\section{Discussion}

In this article, we brought to the forefront the situation in 35 SSA countries of disparity in ORT and continued feeding among under-five children with diarrhea. Our study has been motivated by the SDG's call for disaggregated data on important health indicators like ORT in order to accelerate attainment of the global goals of ensuring universal health coverage in reproductive, maternal, newborn, and child health in 2030 (4). The SDG underscores policy implications of disaggregating health care indicators by dimensions of inequality such as wealth and place of residence. To this end, findings presented in this paper could contribute to the design of equity interventions in order to minimize the observed disparity in one of the most imperative child health care indicators, ORT and continued feeding.

We recorded substantial wealth-related disparity in 20 countries. The disparity was highest and more pronounced in few countries such as Angola, Guinea, Cameroon and Mali. Based on the PAF measure, we showed that the coverage for ORT and continued feeding in 2013 in Nigeria would have been increased, on average, by a staggering nearly $62 \%$ if the country had avoided the poor-rich disparity of the service. This translates to a rise of the coverage from $29-47 \%$. In other words, the coverage gap would have dropped from $71 \mathrm{pp}$ to $53 \mathrm{pp}$. Such big rise in the coverage would significantly have helped the country attain the global goal of universal coverage in maternal and child health care service. Angola, Guinea, and Cameroon each had average wealth related disparity between $24 \%-52 \%$, and as is the case discussed for Nigeria above, their respective national coverage for ORT and continued feeding would have considerably risen if they were able to avoid the observed disparity. Although income disparity in SSA has received greater attention by the SDG (4), there are income disparities that persist still today in the region. In fact, SSA is one of the most unequal regions in the world in terms of income distribution between people (5). Thus, since income is the basis for living, accelerated reduction of poverty in the region could be translated into attaining the other SDGs (5). However, extent of income variations differed by countries in the region. Nigeria is one of the most unequal countries in the region (15), and the disparity continues to cause disparities in health care use like ORT and continued feeding. When there is economic disparity in a nation, already wealthier people are likely to benefit more from the economic growth whereas poor people are left behind with little gains (15). An economic growth would benefit wider public including the poor when countries are of more equal (15). Policies need to focus on correcting the stark income inequality between peoples to rescue poverty related health loss. Due to the fact that income and health have a wellknown close nexuses, equitable economic policy must be regarded as health policy (16), in order to confront the steady economic gradient in health. The mechanisms by which income can exert its influence on health and health cares have been studied (17). One of the possible mechanism through which income affects health and health care is that it increases people's chance of being covered under insurance, and for low-income people, this scheme enhances their means of accessing medical care (17). In 2018, an estimated 27.9 million people were not under insurance coverage in America (18). Since larger share of uninsured people are disproportionately low income families (19), economic policy targeted towards economically lagging households should consider expanding coverage of insurance service. 
We also showed educational inequality in ORT and continued feeding in eleven countries: Angola, Benin, Burundi, Cameron, Chad, Guinea, Guinea Bissau, Kenya, Mali, Mozambique and Nigeria. While the disparity in all these countries requires more work to eliminate the inequality, some countries such as Chad, Guinea, Cameron, Nigeria and Mozambique had the highest average inequality. Had these countries avoided the absolute educational disparity in the use of ORT and continued feeding, then the national coverage of the health service in these countries would have been climbed by, on average, 10 to nearly 18 pp, and the rise in the national coverage would have been increased even more if they had avoided the relative disparity on average by between 26 and 41 percent. Secondary school participation has been most challenging in SSA and South Asia (20). In Chad for instance, more than 6 for every 10 adolescents do not join secondary school. More worse is that, there is striking gaps in level of secondary school participation between income groups, where only less than $50 \%$ of children from poor family are able to attend secondary school in contrast to $80 \%$ of adolescents from the richest household (20). Increasing the proportion of girls who graduated secondary education would greatly contribute to the fight against educated driven inequality in use of ORT and continued feeding in these countries. SSA is required to increase the total enrollments by more than 3 times than the current level if the aim is to attain universal coverage for pre-primary, primary and secondary education by 2030 (21). As an important component of socioeconomic status, education has long been known to influence health disparities directly (22), but education is largely recognized as a legitimate domain of public health action, and is an influential method to disrupt the vicious cycle of poverty. Moreover, education is an essential component of public health interventions that can be used to stimulate health equity and help overcome health disparities (23). Given the current low educational status in most SSA countries, integrating education into public health policies could lead to widespread benefit with respect to challenging health care disparities in the sub-region.

The other interesting finding of this study is that, the largest number of countries in the study did not see sex related disparity around ORT and continued feeding. In 2030, all countries globally are going to ensure gender equality with respect to health care, and participation in politics as well as economic sectors (4). Our study confirms that most SSA countries are likely to attain that goal with respect to this child health care intervention if they do maintain the current momentum. However, there are a handful of countries with slight sex disparity: Niger, Kenya, Guinea-Bissau, and Senegal. In the no sex disparity scenario, Niger would have elevated the coverage of ORT and continued feeding by, on average, 2.3 pp, and female children had a slight advantage over males in use of the service. In Kenya and Guinea-Bissau, on the other hand, male children had on average 6.4 and 11.3 pp respectively more chance of getting the service than female children. Similarly, male children had little but statistically significant advantage over female counterparts in Senegal with regard to receiving ORT and continued feeding. Governments of these countries may consider revising existing interventions in order to make the interventions more gender sensitive and responsive.

No urban-rural inequality was found in 12 of the studied African countries: Ghana, Benin, Burundi, Algeria, Eswatini, Gabon, Gambia, Namibia, Sao Tome, Sierra Leone, Togo and Senegal. In terms of subnational regions, five countries (Comoros, Gabon, Rwanda, Sao Tome and Sierra Leone) managed to distribute the 
service fairly between different regions while the remaining countries had gaps in ORT and continued feeding between different regions. Rural settings and certain geographical areas tend to host rather few children with diarrhea that receive ORT and continued feeding. This further implies that the residence related and subnational regional variations could obstruct SSA countries from attaining of the SDG. To curb the predictable threat of the within country disparities, such countries need to revise existing child health interventions and promote more equitable interventions that target rural areas, poor and illiterate households, and certain geographical areas to accelerate elimination of unacceptable gap in ORT and continued feeding and in so doing could help countries attain the global goal of universal child health care (that is, ORT and continued feeding).

The study has some strength. Findings are produced following transparent and scientifically sound equity analysis technique recommended by the WHO, making the results more reliable for use for decision making. Also, since different summary measures were used in the study, we are hopeful that the disparity was captured from different perspectives. This approach, the approach of adopting 'method-mix', could contribute to not conclude absence of disparity while there is in fact disparity and in doing so could lead to prevention of unsupported decisions. Finally, measuring disparity in ORT and continued feeding through five dimensions of inequality has huge advantage of recognizing population subgroups that suffer lower coverage of the service; policy interventions will then be formulated that favors such groups. The limitation of the study is that, it just highlighted on the disparity of the service across different equity stratifers. However, to better fight against the disparity, it is important to understand the reasons that underlie the observed disparity. In this regard, we recommend future studies to answer this important research question using a decomposition analysis to examine independent contributions of factors that work behind the disparity in ORT and continued feeding.

\section{Conclusions}

In SSA, socioeconomic, urban-rural and geographical inequalities around ORT and continued feeding were substantial. Fortunately, except in a handful of countries, we did not record sex-related inequality in the use of the child health care in the studied countries. Countries need to intensify pro-disadvantaged interventions that target the poor, illiterate, rural residents and some living places in order to challenge the unfair distribution of the service and subsequently markedly decrease the unnecessary diarrheal deaths. Also, the SSA region may benefit more from widespread equitable economic and education policies as income and education have been shown to underlie health care disparities. This could eventually translate into attainment of the global goal of universal coverage for ORT and continued feeding by 2030 .

\section{Abbreviations}

D

Difference

DHS

Demographic and Health Survey 
EA

Enumeration Area

HEAT

Health Equity Assessment Toolkit

ICF

Inner City Fund

ORS

Oral Rehydration Salt/Solution

ORT

Oral Rehydration Therapy

PAF

Population Attributable Fraction

PAR

Population Attributable Risk

PCA

Principal Component Analyses

PSUs

Primary Sampling Units

$\mathrm{R}$

Ratio

SSA

Sub-Saharan Africa

SDG

Sustainable Development Goal

UI

Uncertainty Interval

USAID

United States Agency for International Development

WHO

World Health Organization

\section{Declarations}

Ethics approval and consent to participate

Ethics approval was not required since the data is available to the public domain.

Consent for publication

Not applicable

Availability of data and materials 
The datasets generated and/or analyzed during the current study are available in the WHO's HEAT version 3.1 [https://www.who.int/gho/health_equity/assessment_toolkit/en/].

\section{Competing interests}

The authors declare no competing interests.

\section{Funding}

No funding was received for this article

\section{Authors' contribution}

BZ conceived the study, wrote the result and method; GS contributed to the design and method, wrote the introduction and discussion, and reviewed the manuscript critically for its intellectual content. Both authors approved the final version.

\section{Acknowledgment}

We acknowledge the WHO for distributing the software freely. The fact that the application requires no internet service makes it easy to be used in low-income countries where connection might be a problem.

\section{References}

1. WHO.Diarrhoeal disease. . Updated on May 2, 2017. Accessed on March 26, 2020.

2. GBD 2016 diarrheal diseases collaborators. Estimates of the global, regional and national morbidity, mortality and aetiologies of diarrhea in 195 countries: a systematic analysis for the Global Burden of Disease study 2016. The lancet. 2018;18(11):1211-28.

3. UNICEF. One is too many. Ending child deaths from pneumonia and diarrhea. Every death counts. Nov, 2016. Available from: . Accessed on Feb 23, 2020.

4. General UN, Assembly. Transforming our world: the 2030 Agenda for Sustainable Development, 21 October 2015, A/RES/70/1, available at: [accessed 25 March 2020].

5. Odusola A, Cornia GA, Bhorat H, Concieção P. Income Inequality Trends in sub-Saharan Africa: Divergence, Determinants and Consequences. Overview. UNDP Regional Bureau for Africa. New York: UNDP; 2017.

6. WHO and UNICEF. End preventable deaths: Global Action Plan for Prevention and Control of Pneumonia and Diarrhea. Geneva: WHO; 2013.

7. WHO and UNICE. Clinical management of acute diarrhea. New York: UNICEF and WHO; 2004.

8. WHO. Technical notes. Care seeking for sick children. Available from: . Accessed on Feb 23, 2020.

9. Melinda K, Munos CL, Fischer Walker RE, Black. The effect of oral rehydration solution and recommended home fluids on diarrhea mortality. International Journal of Epidemiology. 2010; 39: 
Pages i75 i87.

10. World Health Organization. Handbook on health inequality monitoring with a special focus on low and middle income countries. Geneva: World Health Organization; 2013.

11. WHO. State of inequality: reproductive, maternal, newborn and child health. Geneva: WHO; 2015.

12. U.S. Department of Health and Human Services. National Institute of Diabetes and digestive and Kidney diseases. Definition \& Facts for Diarrhea. What is diarrhea? Available from: . Accessed on April 6, 2020.

13. Rutstein SO, Johnson K. The DHS wealth index. . Accessed on April 1, 2020.

14. World Health Organization. Health Equity Assessment Toolkit (HEAT): Software for exploring and comparing health inequalities in countries. Built-in database edition. Version 3.1. Geneva: World Health Organization; 2019.

15. THIS DAY. Tackling Economic Inequality. Posted on Sep 6, 2018 [Internet]. Retrieved from: . Accessed on April 6, 2020.

16. Khullar D, Chokshi DA. Health. Income and Poverty: Where We Are \& What Could help. Health Affairs Heal Policy Brief. 2018 Oct. DOI:.

17. Lenhart 0 . The effects of income on health: new evidence from the Earned Income Tax Credit. Review of Economics the Household. 2019: 377-410.

18. Tolbert J, Orgera K, Singer N, Damico A. Key Facts about the Uninsured Population. Issue Brief, December 2019. Retrieved from: . Accessed on April 6, 2020.

19. Gunja MZ, Collins SR. Who are the remaining uninsured, and why do they lack coverage? Findings from the commonwealth fund and Biennial Health Insurance Survey, 2018. 2019.

20. UNICEF.UNICE DATA. Monitoring the situation of children and women. Secondary education. Updated on Oct 2019 [internet]. Retrieved from: . Accessed on a April 10, 2020.

21. UNICEF. Every Child Learns: UNICE Education Strategy 2019-2030. New York: UNICE; 2019.

22. Olshansky SJ, Antonucci T, Berkman L, Binstock RH, Boersch-Supan A, Cacioppo JT, et al. Differences In Life expectancy Due to Race and Educational Differences Are Widening, and Many May Not Catch Up. Health Aff. 2012;31(8):1803-13.

23. Hahn RA, Truman BI. Education Improves Public Health and Promotes Health Equity. International Journal of health services: planning, administration, evaluation.2015; 45(4): 657-678. 